\title{
Atratores para equações de ondas não autônomas com condição de fronteira da acústica
}





\title{
Thales Maier de Souza
}

\section{Atratores para equações de ondas não autônomas com condição de fronteira da acústica}

\author{
Tese apresentada ao Instituto de Ciências \\ Matemáticas e de Computação - ICMC-USP, como \\ parte dos requisitos para obtenção do título de \\ Doutor em Ciências - Matemática. VERSÃO \\ REVISADA. \\ Área de Concentração: Matemática \\ Orientador: Prof. Dr. Ma To Fu
}


Ficha catalográfica elaborada pela Biblioteca Prof. Achille Bassi e Seção Técnica de Informática, ICMC/USP, com os dados fornecidos pelo(a) autor(a)

Maier Souza, Thales
Atratores para equações de ondas não autônomas com
condição de fronteira da acústica / Thales Maier
Souza; orientador Ma To Fu . - São Carlos, 2017.
92 p.
Tese (Doutorado - Programa de Pós-Graduação em
Matemática) -- Instituto de Ciências Matemáticas e
de Computação, Universidade de São Paulo, 2017.
1. Equação da onda. 2. Sistemas dinâmicos não,
Maneares. 3. Condição de fronteira da acústica. I.
To Fu, orient. II. Título.


Thales Maier de Souza

\section{Attractors for non-autonomous wave equations with acoustic boundary condition}

Doctoral dissertation submitted to the Instituto de Ciências Matemáticas e de Computação - ICMCUSP, in partial fulfillment of the requirements for the degree of the Doctorate Program in Mathematics. FINAL VERSION.

Concentration Area: Mathematics

Advisor: Prof. Dr. Ma To Fu

USP - São Carlos

January 2017 



\section{AGRADECIMENTOS}

Agradeço a Deus por me conceder discernimento nos momentos de dificuldades, fazendo com que eu continue caminhando por sua estrada iluminada e, consequentemente, concluindo meus objetivos.

Agradeço aos meus pais, Maier e Eva, por todo apoio que eles me deram nessa caminhada e claro, sem eles eu não seria o que sou hoje.

A minha Irmã, Mayara, pelo incentivo e obviamente, por todos os dias de festas que prestigiamos juntos.

A minha namorada, Rafaella, por todo carinho, ajuda, conselhos, amor, companheirismo, entre muitas outras coisas e que, mesmo à distância, sempre esteve presente cuidando de mim e também, à sua família que me acolheu e me apoiou, em especial a Vó Tatá.

Aos meus amigos, que sempre se mostraram presentes, em especial para Ana, Argola, Badaró, Benedine, Carol, Dione, Dôdô, Fer, Fera, Gi, Mineiro, Mirna, Mostafa, Nelson, Nortio, Paty, Rodrigol, Veto e Zé Wilker, por todo apoio e claro, muito importante, por me concederem o cargo de churrasqueiro oficial.

Ao Ma To Fu, pela excelente orientação e por toda ajuda concedida, e também, pela nossa amizade, que com certeza se manterá tanto no âmbito acadêmico como fora dele.

Agradeço a FAPESP, processo 2013/04648-5, pelo apoio financeiro e institucional. 

"A mente que se abre para uma nova idéia.

Jamais voltará ao seu tamanho original." Albert Einstein 



\section{RESUMO}

MAIER SOUZA, T.. Atratores para equações de ondas não autônomas com condição de fronteira da acústica. 2016. 90 f. Tese (Programa de Doutorado em Matemática) - Instituto de Ciências Matemáticas e de Computação (ICMC/USP), São Carlos - SP.

Esta tese é dedicada ao estudo de uma classe de equações de ondas com condições de fronteira da acústica. Investigamos a dinâmica assintótica de tais equações no caso em que o sistema está sujeito à ação de uma força externa não autônoma. Nessa situação, adicionando uma dissipação fraca, provamos que o problema gera um processo de evolução dissipativo. O nosso objetivo é então o estudo da existência de atratores não autônomos. Num primeiro momento estabelecemos a existência de um atrator do tipo "pullback", minimal, dentro de um universo de conjuntos temperados. Também estudamos a semicontinuidade superior dos atratores quando a perturbação não autônoma tende para zero. Nosso resultado permite considerar forças externas não limitadas e perturbações não lineares com crescimento crítico (de Sobolev). Num segundo momento, fazemos um estudo sobre a existência de atratores uniformes. Em vista de resultados recentes de Zelik (2015), consideramos forças externas mais gerais do que a dita classe das forças compactas por translação (translation-compact). Parte desta tese foi aceita para publicação na revista "Differential and Integral Equations" sob o título "Pullback dynamics of non-autonomous wave equations with acoustic boundary condition".

Palavras-chave: Equação da Onda, Condição de Fronteira da Acústica, Atrator Uniforme, Atrator Pullback. 



\section{ABSTRACT}

MAIER SOUZA, T.. Attractors for non-autonomous wave equations with acoustic boundary condition. 2016. 90 f. Tese (Programa de Doutorado em Matemática) - Instituto de Ciências Matemáticas e de Computação (ICMC/USP), São Carlos - SP.

This thesis is concerned with the study of a class of wave equations with acoustic boundary conditions. We investigate the long-time dynamics of such equations in the case where the system is subject to a non-autonomous external force. In this situation, by adding a weak dissipation, we prove that the problem generates a dissipative evolution process. Our goal is then the existence of non-autonomous attractors. In this direction, we first establishes the existence of a minimal pullback attractor within a universe of tempered sets. We also studied the upper semi-continuity of attractors when the non-autonomous perturbation tends to zero. Our result allows to consider unbounded external forces and nonlinear perturbation with critical (Sobolev) growth. Secondly, we establish the existence of uniform attractors, as well. In view of recent results Zelik (2015) we consider more general external forces than the so called class of translation-compact forces. Part of this thesis was accepted for publication in the journal "Differential and Integral Equations" under the title "Pullback dynamics of non-autonomous wave equations with acoustic boundary condition".

Keywords: Wave Equation, Acoustic Boundary Condition, Uniform Attractor, Pullback Attractor. 



\section{SUMÁRIO}

Introdução

xvii

1 Notações e preliminares 1

2 Teoria de Atratores $\quad 11$

2.1 Introdução . . . . . . . . . . . . . . . . . . . . . . . . . . . 11

2.2 Atratores para semigrupos . . . . . . . . . . . . . . . . 11

2.3 Atrator pullback . . . . . . . . . . . . . . . . . . 13

2.3.1 Critério para compacidade assintótica . . . . . . . . . . . . 16

2.3.2 Semicontinuidade superior de atratores . . . . . . . . . . . . . 18

2.4 Atrator uniforme . . . . . . . . . . . . . . . . . . . . . . . . 19

2.4.1 Forças externas de translação compacta . . . . . . . . . . . . . . 20

2.4.2 Forças externas regulares no espaço . . . . . . . . . . . . . . . . 21

2.4.3 Forças externas regulares no tempo . . . . . . . . . . . . . . . 22

2.4.4 Forças externas: Normal, fracamente normal e fortemente normal 23

2.4.5 Envoltória fraca para forças externas de translação limitada . . . . 24

2.4.6 Processo de evolução e atratores uniformes . . . . . . . . . . . . . 26

2.4.7 Critério para compacidade assintótica . . . . . . . . . . . . 31

3 Atrator pullback para equação da onda com fronteira acústica 35

3.1 Introdução . . . . . . . . . . . . . . . . . . . . . 35

3.2 Hipótese e notações iniciais . . . . . . . . . . . . . . . . . . . 36

3.3 Existência e unicidade . . . . . . . . . . . . . . . . . . . 37

3.4 Desigualdades notáveis . . . . . . . . . . . . . . . . 45 
3.5 Existência do atrator pullback . . . . . . . . . . . . 56

3.6 Semicontinuidade superior . . . . . . . . . . . . . . 61

4 Atrator uniforme para equação da onda com fronteira acústica $\quad 65$

4.1 Introdução . . . . . . . . . . . . . . . . . . . . 65

4.2 Hipóteses e notações preliminares . . . . . . . . . . . . . . 66

4.3 Existência e unicidade . . . . . . . . . . . . . . . . . 66

4.4 Desigualdades notáveis . . . . . . . . . . . . . . . . . . . . . . 69

4.5 Existência do atrator uniforme . . . . . . . . . . . . . . . . . 74 


\section{INTRODUÇÃO}

Nas últimas décadas, tem aumentado consideravelmente as pesquisas científicas envolvendo equações diferenciais parciais, modeladas por problemas provenientes das Engenharias, Física e Biologia. Tais pesquisas têm efetivamente contribuído para o avanço tecnológico e científico da sociedade moderna.

Na presente tese, estudamos a dinâmica assintótica de uma equação da onda, não autônoma, com condição de fronteira da acústica. Adotaremos tanto o ponto de vista da atração pullback assim como o ponto de vista da atração uniforme.

Mais precisamente, seja $\Omega \subset \mathbb{R}^{3}$ um domínio limitado com fronteira regular $\Gamma$ e considere a seguinte equação da onda semilinear não autônoma com condições de fronteira da acústica

$$
\begin{aligned}
& u_{t t}-\Delta u+\omega u_{t}+u+f(u)=h \quad x \in \Omega, t \geq \tau, \\
& \delta_{t t}+\nu \delta_{t}+\delta=-u_{t} \quad x \in \Gamma, t \geq \tau, \\
& \delta_{t}=\partial_{\mathbf{n}} u \quad x \in \Gamma, t \geq \tau,
\end{aligned}
$$

onde $\omega, \nu>0$ são coeficientes de dissipação (damping), $f$ é um termo não linear e $h=h(x, t)$ é uma força externa que depende do tempo. Condições iniciais também são adicionadas ao sistema.

A modelagem física desse tipo de problema consiste em considerar um fluido compreendido em um domínio limitado do qual cada ponto da fronteira vibra como uma mola reagindo a pressão causada pelo fluido, descrita por $-u_{t}$. Sendo $u$ a velocidade potencial do fluido e $\delta$ o deslocamento normal dos pontos da fronteira, este sistema descreve a evolução de $u$, que sofre pequenas perturbações, juntamente com a vibração dos pontos da fronteira. Aqui estamos supondo que a fronteira de $\Omega$ é uma superfície localmente reagente, ou seja, cada ponto da fronteira reage a pressão, causada pelo fluido, de maneira independente uns dos outros. A equação (2) descreve o efeito tipo mola na fronteira que 
interage com - $u_{t}$ e a equação (3) é uma condição de continuidade: a velocidade do deslocamento da fronteira $\delta_{t}$ está compatível com a derivada normal $\partial_{\mathbf{n}} u$, chamada condição de impenetrabilidade da fronteira. Essas duas equações são chamadas condições de fronteira da acústica e foram propostas por Beale e Rosencrans [4].

Nesta tese ao invés de considerarmos o modelo pioneiro, adicionamos um termo não linear $f(u)$ que induz alguns efeitos no movimento da onda, mais ainda, por simplicidade escolhemos todos os coeficientes das equações igual à 1 exceto os coeficientes de damping. Vale ressaltar que, quando $f=-u$ e $\omega=0$, o problema (1)-(3) foi considerado em $[4,5,6]$, onde foram estabelecidos, boa colocação do problema e algumas propriedades espectrais do semigrupo associado.

Este modelo tem sido bastante estudado por vários autores e generalizado de várias formas. A título de exemplo, citamos os trabalhos $[3,5,6,15,21,22,23,25,27,28,30$, 38, 43] e suas referências.

Convém comentar alguns resultados obtidos por Frigeri em [21], onde foi abordado exatamente o problema (1)-(3), porém considerando $h \equiv 0$. Foi estudado o comportamento a longo prazo das soluções desse problema, focando a atenção em propriedades assintóticas globais, tal como a existência de um conjunto absorvente, de um atrator global, de uma certa regularidade da atração e, também, a existência de um atrator exponencial.

Referente ao estudo de sistemas de evolução não autônomos são conhecidas algumas formas de generalizar o conceito de atrator global, por exemplo, tratar o atrator como uma família dependente do tempo, como no caso dos atratores pullback. Uma outra interessante abordagem, do atrator para sistemas não autônomos, possível de se fazer é baseada em, estudar de alguma maneira o sistema dinâmico não autônomo como um sistema dinâmico autônomo e neste caso o atrator será um conjunto que independe do tempo. Esse é o caso dos atratores uniformes. Existem alguns outros tipos de atratores de sistemas dinâmicos não autônomos adaptados para sistema dinâmico aleatório ou estocástico, mais ainda, também existem estudos sobre atratores pullback exponenciais. Para maiores detalhes veja $[10,14,19,20,36]$ e suas referências.

Num primeiro momento, nesta tese, foi considerado uma natural continuação do estudo do Frigeri [21], porém aqui estamos interessados no comportamento a longo prazo de soluções globais de (1)-(3) sobre perturbações de uma força externa dependendo do 
tempo $h=h(x, t)$, que torna o sistema não autônomo. Estudamos a dinâmica pullback desse tipo de problema, em busca de um único $\mathcal{D}$-atrator pullback onde permitimos $f$ com crescimento crítico do tipo $|u|^{3}$ e força externa $h=h(x, t)$ não limitada e dependendo do tempo. A relevância do nosso trabalho está ligada ao fato de que, além de tomar cuidado com as ferramentas envolvendo a teoria de atratores pullback, o estudo da dinâmica pullback desse tipo de problema não tinha sido considerada até então. A teoria de atratores pullback que foi utilizada está baseada nos seguintes trabalhos [10, 26, 41] e suas referências. Os conceitos e resultados utilizados estão disponíveis na Seção 2.3.

Para ser um pouco mais preciso, exibimos a existência de um único $\mathcal{D}$-atrator pullback, primeiramente encontrando um conjunto $\mathcal{D}$-absorvente no sentido pullback, que absorve famílias do universo $\mathcal{D}$ que é formado por família de conjuntos temperados e, em seguida, tratamos da compacidade assintótica no sentido pullback, que foi um dos desafios, já que a fronteira dinâmica causa algumas dificuldades em usar argumentos usuais de potência fracionaria de operadores elípticos, como também observado por Frigeri em [21]. Para contornar tal dificuldade exibimos um critério de compacidade que é adequado para dinâmicas pullback em geral em um contexto de $\mathcal{D}$-atração. Veja Teorema 2.3.11.

Finalizamos o estudo, do ponto de vista da dinâmica pullback, exibindo um certa continuidade de uma familia de atratores com respeito a perturbação da força externa não autônoma, especificamente, como o caráter não autônomo do nosso problema é determinado pela força externa $h(x, t)$, substituímos tal força por $\varepsilon h(x, t)$ e estudamos a semicontinuidade superior dos atratores pullback quando $\varepsilon \rightarrow 0$, sendo que nesse contexto vamos supor um pouco mais de regularidade com relação ao tempo $t$ da força externa $h$, isto é, alguma limitação conveniente.

A coleção desses resultados, boa colocação, existência de um único $\mathcal{D}$-atrator pullback e semicontinuidade superior são autênticas e juntas resultaram em um artigo submetido. Veja [34].

Como dito a pouco, uma outra interessante abordagem de um sistema de evolução não autônomo é fazer uso da teoria de atratores uniformes. Nesta direção existem vários trabalhos que abordam o tema atrator uniforme e, também, fazem aplicações da teoria em equações da onda. Veja $[45,14,29,32]$.

Para contextualizar o tema atrator uniforme, faremos alguns breves comentários e que serão expostos com mais detalhes na Seção 2.4 e no Capítulo 4. 
Note que o problema (1)-(3) depende da força externa $h$. No contexto da teoria de atratores uniformes primeiramente vamos considerar um problema não autônomo da seguinte forma

$$
\begin{aligned}
& u_{t t}-\Delta u+\omega u_{t}+u+f(u)=g(t) \quad \text { sobre } \Omega, t \geq \tau, \\
& \delta_{t t}+\nu \delta_{t}+\delta=-u_{t} \quad \text { sobre } \quad \Gamma, t \geq \tau, \\
& \delta_{t}=\partial_{\mathbf{n}} u \text { sobre } \quad \Gamma, t \geq \tau
\end{aligned}
$$

definido num espaço de fase $\mathcal{H}$ (à definir) e supondo $g$ uma força externa inicial dependendo do tempo. Considere

$$
\Sigma_{0}=\{\text { translações no tempo de } g\}
$$

e defina a envoltória $\mathcal{H}(g)$ da força inicial $g$ com sendo o fecho de $\Sigma_{0}$ em uma topologia conveniente. Estas translações no tempo são conhecidas como "shifts". Assim podemos definir uma nova família de problemas da seguinte forma

$$
\begin{aligned}
& u_{t t}-\Delta u+\omega u_{t}+u+f(u)=h \quad x \in \Omega, t \geq \tau, h \in \mathcal{H}(g) \\
& \delta_{t t}+\nu \delta_{t}+\delta=-u_{t} \quad x \in \Gamma, t \geq \tau \\
& \delta_{t}=\partial_{\mathbf{n}} u \quad x \in \Gamma, t \geq \tau
\end{aligned}
$$

Supondo que este problema acima é globalmente bem posto em $\mathcal{H}$, como veremos na Seção 4.3, concluímos que, através do operador solução, para cada $h \in \mathcal{H}(g)$, podemos definir um processo de evolução

$$
U_{h}(t, \tau): \mathcal{H} \rightarrow \mathcal{H}
$$

Assim, denotando por $X=\mathcal{H} \times \mathcal{H}(g)$, chamado espaço de fase extendido, podemos definir um semigrupo extendido $\mathbb{S}(t): X \rightarrow X$ da seguinte forma,

$$
\mathbb{S}(t)\left(z_{0}, h\right)=\left(U_{h}(t, 0) z_{0}, T(t) h\right)
$$

onde $z_{0} \in \mathcal{H}, h \in \mathcal{H}(g)$ e

$$
[T(s) h](t)=h(t+s)
$$

No caso em que este semigrupo $\mathbb{S}(\cdot)$ possui um atrator global $\mathbb{A}$ em $X$ dizemos que a projeção na primeira coordenada de $X$, digamos $\mathcal{A}=\pi_{1} \mathbb{A}$, é o chamado atrator uniforme associado ao problema (7)-(9). 
É fácil ver que essa construção acima depende claramente da topologia escolhida no espaço de fase estendido. Segundo Chepyzhov e Vishik [14], existem duas formas naturais de escolher tais topologias. A primeira é considerar a topologia fraca em ambas coordenadas do espaço $X$ e lidar com atratores uniformes fracos e a segunda é considerar a topologia forte em ambas as coordenadas e lidar com atratores uniformes fortes. Note que a topologia da envoltória depende da classe da força externa inicial $g$. Contudo, na Seção 2.4 e no Capítulo 4, vamos abordar o caso em que temos topologia forte em $\mathcal{H}$ e topologia fraca na envoltória $\mathcal{H}(g)$ e utilizaremos recentes resultados de Zelik [45] para garantir existência de atrator uniforme forte.

Conforme Zelik, vamos apresentar uma classe nova de forças externas que não são de translação compacta, porem ainda permanecem na classe das funções de translação limitadas, e que ainda garante a existência de atratores uniformes. A abordagem de Zelik é feita levando em consideração que as forças externas de translação compacta podem ser aproximadas por funções que são suaves com respeito ao espaço e o tempo. Seguindo essa idéia surge a classe das forças externas regulares no tempo que consiste de funções de translação limitada que podem ser aproximadas por funções suaves no tempo. Vamos formalizar tais conceitos na Seção 2.4. Veja também [45].

Como veremos na Seção 2.4, convém observar que se força externa inicial $g$ é de translação compacta a topologia na envoltória $\mathcal{H}(g)$ será naturalmente a topologia forte e já no caso de a força externa inicial $g$ for de translação limitada a topologia na envoltória será a topologia fraca.

Numa segunda parte dessa tese, mostramos a existência de um atrator uniforme fraco do problema (7)-(9) no caso em que a força externa inicial $g$ é de translação limitada e ao supor que $g$ seja regular no tempo usando alguns resultados e argumentos similares aos de Zelik [45] concluímos a existência de um atrator uniforme forte para o problema (7)-(9). Aqui também foi necessário um critério de compacidade assintótica uniforme análogo ao critério de compacidade no caso da dinâmica pullback, veja Seção 2.4.7. 



\section{Notações e preliminares}

Neste Capítulo iremos introduzir algumas notações e apresentar alguns resultados clássicos para facilitar a compreensão do conteúdo abordado e com intuito de tornar essa tese a mais autossuficiente possível. Alguns resultados serão apresentados sem uma prova formal, porém forneceremos referências clássicas para os mesmos.

Considere $\Omega$ um conjunto do $\mathbb{R}^{n}$, aberto e limitado, com fronteira $\Gamma$ suave que denominamos por domínio do $\mathbb{R}^{n}$. Neste caso a fronteira $\Gamma$ é uma variedade compacta, sem bordo, $C^{\infty}$ e de dimensão $n-1$.

Denotamos $L^{p}(\Omega)$ com $1 \leq p<\infty$, o espaço de Banach das funções mensuráveis $u: \Omega \rightarrow \mathbb{R}$ tais que $|u|^{p}$ é Lebesgue integrável sobre $\Omega$, cuja norma é dada por

$$
\|u\|_{L^{p}(\Omega)}=\left(\int_{\Omega}|u(x)|^{p} d x\right)^{\frac{1}{p}} .
$$

Por $L^{\infty}(\Omega)$ denotamos o espaço de Banach das funções mensuráveis $u: \Omega \rightarrow \mathbb{R}$ que são essencialmente limitadas em $\Omega$. A norma em $L^{\infty}(\Omega)$ é

$$
\|u\|_{L^{\infty}(\Omega)}=\operatorname{supess}_{x \in \Omega}|u(x)|
$$

Em particular, quando $p=2, L^{2}(\Omega)$ é um espaço de Hilbert cujo produto interno é dado por

$$
(u, v)_{L^{2}(\Omega)}=\int_{\Omega} u(x) v(x) d x
$$

De modo análogo ao $L^{2}(\Omega)$, denotamos por $L^{2}(\Gamma)$ o espaço de Hilbert das funções mensuráveis $u: \Gamma \rightarrow \mathbb{R}$ tais que $|u|^{2}$ é Lebesgue integrável em $\Gamma$, cujo produto interno e norma são

$$
(u, v)_{L^{2}(\Gamma)}=\int_{\Gamma} u(x) v(x) d x \quad \text { e } \quad\|u\|_{L^{2}(\Gamma)}=\left(\int_{\Gamma}|u(x)|^{2} d x\right)^{\frac{1}{2}}
$$


Por $\mathcal{D}(\Omega)$ denotamos o espaço das funções testes sobre $\Omega$, isto é, $\mathcal{D}(\Omega)$ é o espaço das funções infinitamente diferenciáveis com suporte compacto em $\Omega, C_{0}^{\infty}(\Omega)$, munido com a topologia do limite indutivo.

Denotamos por $\mathcal{D}^{\prime}(\Omega)$ o espaço vetorial das distribuições sobre $\Omega$, munido com a topologia fraca. Observe que, dado $T \in \mathcal{D}^{\prime}(\Omega)$ e $\alpha$ um multi-índice, definimos a derivada da ordem $\alpha$, da distribuição $T$, por

$$
\left\langle D^{\alpha} T, \varphi\right\rangle=(-1)^{|\alpha|}\left\langle T, D^{\alpha} \varphi\right\rangle
$$

para todo $\varphi \in \mathcal{D}(\Omega)$. É possível verificar que $D^{\alpha} T$ é uma distribuição e, mais ainda, que o operador $D^{\alpha}: \mathcal{D}^{\prime}(\Omega) \rightarrow \mathcal{D}^{\prime}(\Omega)$, que associa cada $T \in \mathcal{D}^{\prime}(\Omega)$ com a distribuição $D^{\alpha} T \in \mathcal{D}^{\prime}(\Omega)$, é linear e contínuo.

Representa-se por $W^{m, p}(\Omega)$ o espaço vetorial de todas a funções $u \in L^{p}(\Omega)$ tais que $D^{\alpha} u$ pertence à $L^{p}(\Omega)$ para todo multi-índice satisfazendo $|\alpha| \leq m$. O espaço $W^{m, p}(\Omega)$ munido da norma

$$
\|u\|_{W^{m, p}(\Omega)}=\left\{\begin{array}{l}
\left(\sum_{|\alpha| \leq m} \int_{\Omega}\left|D^{\alpha} u(x)\right|^{p} d x\right)^{\frac{1}{p}}, \quad \text { para } 1 \leq p<\infty, \\
\sum_{|\alpha| \leq m} \operatorname{supess}_{x \in \Omega}\left|D^{\alpha} u(x)\right|, \quad \text { para } \quad p=\infty,
\end{array}\right.
$$

é um espaço de Banach. No caso em que $p=2$, denotamos $W^{m, 2}(\Omega)$ por $H^{m}(\Omega)$. Note que os espaços de Sobolev $H^{m}(\Omega)$ são espaços de Hilbert, onde o produto interno é dado por

$$
(u, v)_{H^{m}(\Omega)}=\sum_{|\alpha| \leq m}\left(D^{\alpha} u, D^{\alpha} v\right)_{L^{2}(\Omega)} .
$$

Sabe-se que $C_{0}^{\infty}(\Omega)$ é denso em $L^{p}(\Omega)$, mas não é verdade que $C_{0}^{\infty}(\Omega)$ é denso em $W^{m, p}(\Omega)$ para $m \geq 1$. Motivado por este fato define-se o espaço $W_{0}^{m, p}(\Omega)$ como sendo o fecho de $C_{0}^{\infty}(\Omega)$ em $W^{m, p}(\Omega)$, isto é, $\overline{C_{0}^{\infty}(\Omega)} W^{m, p}(\Omega)=W_{0}^{m, p}(\Omega)$.

Suponha que $\Omega$ é um aberto e limitado em alguma direção $x_{i}$ do $\mathbb{R}^{n}$ e $1 \leq p<\infty$, então podemos definir em $W_{0}^{m, p}(\Omega)$ a seguinte norma

$$
\|u\|_{W_{0}^{m, p}(\Omega)}=\left(\sum_{|\alpha|=m} \int_{\Omega}\left|D^{\alpha} u(x)\right|^{p} d x\right)^{\frac{1}{p}} .
$$

Note que esta norma é equivalente a norma $\|u\|_{W^{m, p}(\Omega)}$. 
Suponha que $1 \leq p<\infty$ e $1 \leq q<\infty$ tal que $\frac{1}{p}+\frac{1}{q}=1$. Representa-se por $W^{-m, q}(\Omega)$ o dual topológico de $W_{0}^{m, p}(\Omega)$ e o dual topológico de $H_{0}^{m}(\Omega)$ é denotado por $H^{-m}(\Omega)$.

Ainda sobre os espaços de Sobolev, agora, de ordem $s \in \mathbb{R}^{+}$. Denotamos $H^{s}\left(\mathbb{R}^{n}\right)=$ $\left\{u \in S^{\prime} ;\left(1+\|x\|^{2}\right)^{\frac{s}{2}} \hat{u} \in L^{2}\left(\mathbb{R}^{n}\right)\right\}$ o espaço de Hilbert munido com o seguinte produto interno

$$
(u, v)_{H^{s}\left(\mathbb{R}^{n}\right)}=\int_{\mathbb{R}^{n}}\left(1+\|x\|^{2}\right)^{s} \hat{u}(x) \hat{v}(x) d x .
$$

Aqui, $\hat{u}$ indica a transformada de Fourier de $u$ definida, para funções $u \in L^{1}\left(\mathbb{R}^{n}\right)$, por

$$
\hat{u}(x)=(2 \pi)^{-\frac{n}{2}} \int_{\mathbb{R}^{n}} e^{-i(x, y)_{\mathbb{R}^{n}}} u(y) d y .
$$

Além disso, se $s \geq 0$ temos que $H^{-s}\left(\mathbb{R}^{n}\right)=\left(H^{s}\left(\mathbb{R}^{n}\right)\right)^{\prime}$ e $H^{s}\left(\mathbb{R}^{n}\right) \hookrightarrow L^{2}\left(\mathbb{R}^{n}\right) \hookrightarrow H^{-s}\left(\mathbb{R}^{n}\right)$. Considere a seguinte aplicação:

$$
\begin{aligned}
r_{\Omega}: L^{2}\left(\mathbb{R}^{n}\right) & \longrightarrow L^{2}(\Omega) \\
u & \longmapsto r_{\Omega}(u)=\left.u\right|_{\Omega}
\end{aligned}
$$

que leva $u$ na sua restrição à $\Omega$. Para $s \geq 0$ definimos $H^{s}(\Omega)=\left\{u_{\left.\right|_{\Omega}} ; u \in H^{s}\left(\mathbb{R}^{n}\right)\right\}$. A fim de definir uma topologia em $H^{s}(\Omega)$ consideremos o seguinte espaço de Banach

$$
\frac{H^{s}\left(\mathbb{R}^{n}\right)}{\operatorname{ker}\left(r_{\Omega}\right)}=\left\{v+\operatorname{ker}\left(r_{\Omega}\right) ; v \in H^{s}\left(\mathbb{R}^{n}\right)\right\}=\left\{[v] ; v \in H^{s}\left(\mathbb{R}^{n}\right)\right\}
$$

munido da norma

$$
\|[v]\|=\inf \left\{\|w\|_{H^{s}\left(\mathbb{R}^{n}\right)} ; w \in[v]\right\}=\inf \left\{\|w\|_{H^{s}\left(\mathbb{R}^{n}\right)} ; w \in v+\operatorname{ker}\left(r_{\Omega}\right)\right\} .
$$

Assim, para $s \geq 0$ e $u \in H^{s}(\Omega)$ definimos

$$
\|u\|_{H^{s}(\Omega)}=\|[v]\|,
$$

onde $u=r_{\Omega}(v)$ para algum $v \in H^{s}\left(\mathbb{R}^{n}\right)$. Observe que $H^{s}(\Omega)$ é um espaço de Hilbert munido com essa norma, e, além disso, para $s \in \mathbb{N}$ as normas

$$
\|u\|_{W^{s, 2}(\Omega)}=\left(\sum_{|\alpha| \leq m} \int_{\Omega}\left|D^{\alpha} u(x)\right|^{2} d x\right)^{\frac{1}{2}} \text { e }\|u\|_{H^{s}(\Omega)}=\inf \left\{\|w\|_{H^{s}\left(\mathbb{R}^{n}\right)} ; r_{\Omega}(w)=u\right\},
$$

são equivalentes em $H^{s}(\Omega)$. Também denotamos $H^{-s}(\Omega)=\left(H_{0}^{s}(\Omega)\right)^{\prime}$ onde $H_{0}^{s}(\Omega)=$ $\overline{\mathcal{D}}(\Omega)^{H^{s}(\Omega)}$. Para maiores detalhes consulte $[1,11]$. 
Vamos definir os espaços de Sobolev sobre a variedade $\Gamma$, fronteira de $\Omega$. Inicialmente, suponha que $\Omega=\mathbb{R}_{+}^{n}$, neste caso $\Gamma=\left\{\left(x^{\prime}, 0\right) ; x^{\prime} \in \mathbb{R}^{n-1}\right\}$ e identificamos toda função real $u$ definida em $\Gamma$ com a função $x^{\prime} \rightarrow u\left(x^{\prime}, 0\right)$ do $\mathbb{R}^{n-1}$ em $\mathbb{R}$. Com tal identificação temos que $\mathcal{D}(\Gamma)=\mathcal{D}\left(\mathbb{R}^{n-1}\right)$ e $L^{p}(\Gamma)=L^{p}\left(\mathbb{R}^{n-1}\right)$. Portanto, neste caso mais simples, definimos $H^{s}(\Gamma)$ como sendo $H^{s}\left(\mathbb{R}^{n-1}\right)$.

Por outro lado, quando $\Omega$ é um aberto e limitado do $\mathbb{R}^{n}$ com fronteira suave $\Gamma$, fixamos um sistema de cartas locais de $\Gamma$, isto é, $\left\{\left(U_{1}, \psi_{1}\right), \ldots,\left(U_{N}, \psi_{N}\right)\right\}$ e funções teste $\sigma_{1}, \ldots, \sigma_{N}$ no $\mathbb{R}^{n}$ tais que $\operatorname{supp}\left(\sigma_{j}\right) \subset U_{j}, j=1,2, \ldots, N$ tal que $\sum \sigma_{j}(x)=1$ para todo $x \in \Gamma$. Assim dada uma função $w$ definida sobre $\Gamma$, para todo $j=1,2, \ldots, N$ defina

$$
w_{j}(y)=\left\{\begin{array}{l}
\sigma_{j} \circ w\left(\psi_{j}^{-1}\left(y^{\prime}, 0\right)\right), \quad \text { se } \quad y \in \Omega_{0}=(0,1)^{n-1} \\
0, \quad \text { se } \quad y^{\prime} \in\left[\mathbb{R}^{n-1}-\Omega_{0}\right]
\end{array}\right.
$$

Dado $s>0$, então $H^{s}(\Gamma)$ é o espaço de Hilbert das funções $w$ definidas em $\Gamma$ tais que $w_{j} \in H^{s}\left(\mathbb{R}^{n-1}\right)$ para todo $j=1,2, \ldots, N$ e munido com o seguinte produto interno:

$$
(w, v)_{H^{s}(\Gamma)}=\sum_{j=1}^{N}\left(w_{j}, v_{j}\right)_{H^{s}\left(\mathbb{R}^{n-1}\right)}
$$

para todo $w, v \in H^{s}(\Gamma)$.

Observe que, considerando $\mathcal{D}(\bar{\Omega})$ com a topologia induzida do espaço $H^{1}(\Omega)$ a aplicação $\gamma_{0}: \mathcal{D}(\bar{\Omega}) \rightarrow H^{\frac{1}{2}}(\Gamma)$ é linear e contínua. Sendo $\mathcal{D}(\bar{\Omega})$ denso em $H^{1}(\Omega)$, temos que esta aplicação se estende por densidade a uma aplicação linear e contínua, que ainda representaremos por $\gamma_{0}$, de $H^{1}(\Omega)$ em $H^{\frac{1}{2}}(\Gamma)$, satisfazendo a seguinte condição:

$$
\gamma_{0} u=u_{\left.\right|_{\Gamma}}, \quad \text { para todo } \quad u \in \mathcal{D}(\bar{\Omega})
$$

a qual denomina-se aplicação traço de ordem zero. Note que a aplicação traço $\gamma_{0}$ : $H^{1}(\Omega) \rightarrow H^{\frac{1}{2}}(\Omega)$ é sobrejetiva e o núcleo de $\gamma_{0}$ é o espaço $H_{0}^{1}(\Omega)$.

Seja $\mathbf{n}$ o vetor normal unitário exterior em $\Gamma$. Para todo $u \in \mathcal{D}(\bar{\Omega})$, considere $\gamma_{1} u=\partial_{\mathbf{n}} u_{\left.\right|_{\Gamma}}$ a derivada normal de $u$. Assim, munindo $\mathcal{D}(\bar{\Omega})$ com a topologia induzida de $H^{2}(\Omega)$ a aplicação $\gamma_{1}: \mathcal{D}(\bar{\Omega}) \rightarrow H^{\frac{1}{2}}(\Gamma)$ é linear e contínua e portanto se estende por densidade a uma aplicação linear e contínua $\gamma_{1}: H^{2}(\Omega) \rightarrow H^{\frac{1}{2}}(\Gamma)$ a qual denomina-se aplicação traço de ordem 1.

Denotamos $\mathcal{H}(\Delta, \Omega)=\left\{u \in H^{1}(\Omega) ; \Delta u \in L^{2}(\Omega)\right\}$, o espaço de Hilbert munido com o produto interno

$$
(u, v)_{\mathcal{H}(\Delta, \Omega)}=(u, v)_{H^{1}(\Omega)}+(\Delta u, \Delta v)_{L^{2}(\Omega)},
$$


para todo $u, v \in \mathcal{H}(\Delta, \Omega)$. Note que $H^{2}(\Omega) \subsetneq \mathcal{H}(\Delta, \Omega)$, entretanto, ainda podemos definir o traço de ordem 1 para funções de $\mathcal{H}(\Delta, \Omega)$ num sentido mais fraco. Munindo $\mathcal{D}(\bar{\Omega})$ com a topologia induzida de $\mathcal{H}(\Delta, \Omega)$, concluímos que a aplicação $\gamma_{1}: \mathcal{D}(\bar{\Omega}) \rightarrow H^{-\frac{1}{2}}(\Gamma)$ é linear e contínua e se estende por densidade a uma única aplicação linear e contínua $\gamma_{1}: \mathcal{H}(\Delta, \Omega) \rightarrow H^{-\frac{1}{2}}(\Gamma)$.

Teorema 1.0.1 (Fórmula de Gauss e a Fórmula de Green). Seja $\Omega$ um aberto, limitado e suave do $\mathbb{R}^{n}$.

i) Se $u, v \in H^{1}(\Omega)$, temos a fórmula de Gauss:

$$
\int_{\Omega} u \frac{\partial v}{\partial x_{i}} d x=-\int_{\Omega} \frac{\partial u}{\partial x_{i}} v d x+\int_{\Gamma} \gamma_{0}(u) \gamma_{0}(v) \mathbf{n}_{i} d \gamma
$$

para todo $i=1, \ldots, n$. Observe que, $\mathbf{n}=\left(\mathbf{n}_{1}, \ldots, \mathbf{n}_{n}\right)$ denota o vetor normal unitário exterior à $\Gamma$.

ii) Se $u \in H^{2}(\Omega)$ e $v \in H^{1}(\Omega)$, temos a fórmula de Green:

$$
\int_{\Omega} \nabla u \nabla v d x=-\int_{\Omega} \Delta u v d x+\int_{\Gamma} \gamma_{1}(u) \gamma_{0}(v) d \gamma
$$

Teorema 1.0.2 (Fórmula de Green generalizada). Seja $\Omega$ um aberto, limitado e suave do $\mathbb{R}^{n}$. Se $u \in \mathcal{H}(\Delta, \Omega)$ e $v \in H^{1}(\Omega)$ então,

$$
\int_{\Omega} \Delta u v d x+\int_{\Omega} \nabla u \nabla v d x=\langle u, v\rangle_{H^{-1 / 2}(\Gamma) \times H^{1 / 2}(\Gamma)} .
$$

Observação 1.0.3. Para simplificar a notação vamos omitir as funções $\gamma_{1}$ e $\gamma_{0}$ ao integrarmos funções sobre a fronteira.

Neste momento iremos definir alguns espaços que serão frequentemente utilizados. Considere $L^{p}(0, T ; X)$, onde $X$ é um espaço de Banach e $1 \leq p<\infty$, o conjunto das funções vetoriais mensuráveis $u:(0, T) \rightarrow X$, tais que $\|u(t)\|_{X}$ pertence ao espaço $L^{p}(0, T)$. O conjunto $L^{p}(0, T ; X)$ é um espaço de Banach munido com a norma

$$
\|u\|_{L^{p}(0, T ; X)}=\left(\int_{0}^{T}\|u(t)\|_{X}^{p} d t\right)^{\frac{1}{p}} .
$$

Quando $p=\infty$, então $L^{\infty}(0, T ; X)$ é um espaço de Banach, formado pelas funções vetoriais mensuráveis $u:(0, T) \rightarrow X$, tais que $\|u(t)\|_{X}$ pertence a $L^{\infty}(0, T)$, munido com a norma

$$
\|u\|_{L^{\infty}(0, T ; X)}=\operatorname{supess}_{t \in(0, T)}\|u(t)\|_{X}
$$


Definimos também o espaço da funções vetoriais $p$-localmente integráveis, denotado por $L_{l o c}^{p}(\mathbb{R} ; X)$, que é formado por todas as funções vetoriais $u: \mathbb{R} \rightarrow X$ tais que para cada intervalo $\left[t_{1}, t_{2}\right] \subset \mathbb{R}$ tem-se que $u \in L^{p}\left(t_{1}, t_{2} ; X\right)$.

A seguir exibiremos alguns resultados notáveis e algumas desigualdades. Para maiores detalhes veja $[1,7,11,12]$. Começamos com um Teorema de imersão de Sobolev.

Teorema 1.0.4 (Imersões de Sobolev). Seja $\Omega \subset \mathbb{R}^{N}$ um domínio limitado com fronteira suave.

(i) Se $m p<N$, então a seguinte inclusão é contínua

$$
W^{m, p}(\Omega) \hookrightarrow L^{q^{*}}(\Omega), \text { onde } \frac{1}{q^{*}}=\frac{1}{p}-\frac{m}{N} .
$$

Além disso, a inclusão é compacta para qualquer $q$, tal que $1 \leq q<q^{*}$.

(ii) Se $m p=N$, então a seguinte inclusão é continua e compacta

$$
W^{m, p}(\Omega) \stackrel{c}{\hookrightarrow} L^{q}(\Omega), \text { para todo } 1 \leq q<\infty
$$

Além disso, se $p=1$ e $m=N$, então vale a mesma relação acima quando $q=\infty$.

(iii) Se $k+1>m-\frac{N}{p}>k, k \in \mathbb{N}$, então escrevendo $m-\frac{N}{p}=k+\alpha$, para $0<\alpha<1$ temos que a seguinte inclusão é contínua

$$
W^{m, p}(\Omega) \hookrightarrow C^{k, \alpha}(\bar{\Omega}),
$$

onde $C^{k, \alpha}(\bar{\Omega})$ representa o espaço das funções em $C^{k}(\bar{\Omega})$ cujas derivadas de ordem $k$ são $\alpha$-Holder contínuas. Além disso, se $N=m-k-1, \alpha=1$ e $p=1$ tem-se que a inclusão acima e a inclusão $W^{m, p}(\Omega) \hookrightarrow C^{k, \beta}(\bar{\Omega})$ é compacta para todo $0 \leq \beta<\alpha$.

Teorema 1.0.5 (Desigualdade de Hölder Generalizada). Sejam $1 \leq p_{i} \leq \infty$, para todo $i=1,2, \ldots, n$, tais que

$$
\frac{1}{p_{1}}+\frac{1}{p_{2}}+\ldots+\frac{1}{p_{n}}=\frac{1}{r} \leq 1 .
$$

Nessas condições, se $f_{i} \in L^{p_{i}}(\Omega)$ então $f:=\prod_{i=1}^{n} f_{i} \in L^{r}(\Omega)$ e, mais ainda,

$$
\|f\|_{r} \leq \prod_{i=1}^{n}\left\|f_{i}\right\|_{p_{i}}
$$


Lema 1.0.6 (Desigualdade de Young com $\epsilon$ ). Sejam $1<p, q<\infty \operatorname{com} \frac{1}{p}+\frac{1}{q}=1 e$ qualquer $\epsilon>0$. Então,

$$
a b \leq \epsilon a^{p}+C(\epsilon) b^{q}, \quad \text { para todos } a, b \geq 0,
$$

onde $C(\epsilon)=(\epsilon p)^{-\frac{q}{p}} q^{-1}$.

Lema 1.0.7 (Desigualdade de Gronwall). Sejam $\alpha \geq 0$ uma constante, $\beta \in L^{1}(a, b) e$ $\phi \in L^{\infty}(a, b)$ tais que $\beta>0$ e $\phi>0$. Sob essas condições, se

$$
\phi \leq \alpha+\int_{a}^{b} \beta(s) \phi(s) d s, \quad \text { para todo } t \in[a, b] .
$$

Então

$$
\phi(t) \leq \alpha e^{\int_{a}^{b} \beta(s) d s}, \quad \text { para todo } \quad t \in[a, b]
$$

O próximo resultado exibirá condições para que um conjunto limitado seja relativamente compacto. A prova desse teorema pode ser encontrada em [40].

Teorema 1.0.8 (Teorema de Aubin-Lions). Assuma que $X, Y$ e $Z$ são três espaços de Banach satisfazendo as seguintes inclusões $X \stackrel{c}{\hookrightarrow} Y \hookrightarrow Z$.

- Se F é um conjunto limitado em $L^{p}(a, b ; X)$, para algum $p \in[1, \infty)$, tal que o conjunto $\partial_{t} F:=\left\{\partial_{t} f ; f \in F\right\}$ é limitado em $L^{q}(a, b ; Z)$, para algum $q \geq 1$. Então $F$ é relativamente compacto em $L^{p}(a, b ; Y)$. Mais ainda, se $q>1$, então $F$ é também relativamente compacto em $C(a, b ; Z)$. Aqui $\partial_{t} f$ significa a derivada no sentido das distribuições.

- Se F é um conjunto limitado em $L^{\infty}(a, b ; X)$ e o conjunto $\partial_{t} F$ é limitado em $L^{r}(a, b ; Z)$, para algum $r>1$, então $F$ é relativamente compacto em $C(a, b ; Y)$.

Teorema 1.0.9 (Teorema de Lax-Milgram). Seja $\left(H,(\cdot, \cdot)_{H}\right)$ um espaço de Hilbert e $a(u, v)$ uma forma bilinear contínua e coerciva. Então para todo funcional $\varphi \in H^{\prime}$, existe um único $u \in H$ tal que

$$
a(u, v)=\langle\varphi, v\rangle_{H^{\prime}, H}, \quad \text { para todo } \quad v \in H .
$$

Além disso, se $a(u, v)$ é simétrica, então $u \in H$ é tal que

$$
\frac{1}{2} a(u, u)-\langle\varphi, u\rangle_{H^{\prime}, H}=\min _{v \in H}\left\{\frac{1}{2} a(v, v)-\langle\varphi, v\rangle_{H^{\prime}, H}\right\} .
$$


Teorema 1.0.10. Seja $X$ um espaço de Banach reflexivo. Se $B \subset X$ é limitado, então $B$ é compacto na topologia fraca $\sigma\left(X, X^{\prime}\right)$, ou seja, qualquer sequência $\left\{x_{n}\right\} \subset B$ possui uma subsequência $\left\{x_{n_{k}}\right\}$ convergente em $X$ na topologia fraca $\sigma\left(X, X^{\prime}\right)$.

Teorema 1.0.11. Seja X um espaço de Banach separável. Se $F \subset X^{\prime}$ é limitado, então $F$ é compacto em $X$ na topologia fraca estrela $\sigma\left(X^{\prime}, X\right)$, isto é, qualquer sequência $\left\{f_{n}\right\} \subset F$ possui uma subsequência convergente em $X^{\prime}$ na topologia fraca estrela $\sigma\left(X^{\prime}, X\right)$.

Teorema 1.0.12. Seja $A: D(A) \subset H \rightarrow H$ um operador maximal e monótono de um espaço de Hilbert $H$. Então para todo $u_{0} \in D(A)$ existe uma única função

$$
u \in C^{1}([0, \infty) ; H) \cap C^{0}([0, \infty) ; D(A)),
$$

de modo que

$$
\begin{aligned}
& \frac{d u}{d t}(t)+A u=0, \quad \text { para todo } t>0, \\
& u(0)=u_{0}
\end{aligned}
$$

Além disso, verifica-se as seguintes estimativas,

$$
\|u(t)\|_{H} \leq\left\|u_{0}\right\|_{H} \quad e \quad\left\|\frac{d u}{d t}(t)\right\|_{H}=\|A u(t)\|_{H} \leq\left\|A u_{0}\right\|_{H},
$$

para todo $t \geq 0$.

Teorema 1.0.13. O operador $A-\omega I$ é gerador infinitesimal de um semigrupo $S$, de classe $C^{0}$, satisfazendo $\|S(t)\| \leq M$ se, e somente se $A$ é gerador infinitesimal de um semigrupo $S$, de classe $C^{0}$, satisfazendo $\|S(t)\| \leq M e^{\omega t}$.

Teorema 1.0.14 (Teorema de Lummer-Phillips). Seja $A: D(A) \subset H \rightarrow H$ um operador linear do espaço de Hilbert $H$, densamente definido. Se $A$ é dissipativo e existe $\lambda_{0}>0$ tal que $\operatorname{Im}\left(\lambda_{0} I-A\right)=H$, então A é gerador infinitesimal de um semigrupo de contrações $C^{0}$ sobre $H$.

Teorema 1.0.15. Suponha que A é um operador maximal e monótono sobre um espaço de Hilbert $H$ e que $0=A 0$. Assumindo $u_{0} \in D(A), f \in W^{1,1}(0, t ; H)$ para todo $t>0$ e $B: H \rightarrow H$ é uma aplicação localmente Lipschitz, isto é,

$$
\|B u-B v\|_{H} \leq L(K)\|u-v\|_{H},
$$


sempre que $\|u\|,\|v\| \leq K$. Então existe $t_{\max } \leq \infty$ tal que o seguinte problema

$$
\begin{aligned}
& u_{t}+A u+B u=f, \\
& u(0)=u_{0} \in H,
\end{aligned}
$$

tem uma única solução forte no intervalo $\left[0, t_{\max }\right)$, isto é, $u \in W^{1, \infty}\left(0, t_{\max } ; H\right)$ e $u(t) \in$ $D(A)$ para todo $t \in\left[0, t_{\max }\right)$.

Mais ainda, se assumirmos apenas que $u_{0} \in \overline{D(A)}$ e que $f \in L^{1}(0, t ; H)$ para todo $t>0$, obtemos uma única solução generalizada $u \in C\left(\left[0, t_{\max }\right) ; H\right)$ do problema acima (generalizada, no sentido de que u é limite de soluções fortes, soluções com dado inicial no dominio de $A$ ).

Em ambos os casos temos que $\lim _{t \rightarrow t_{\max }}\|u(t)\|=\infty$ sempre que $t_{\max }<\infty$.

Vamos finalizar esta seção com um pouco sobre o operador de Nemytskii que será utilizado no estudo do expoente crítico. Veja [39]. Começamos com a definição das condições de Carathéodory de uma função.

Definição 1.0.16. Seja $\Omega \subset \mathbb{R}^{n}$ um domínio. Dizemos que uma função

$$
\Omega \times \mathbb{R}^{m} \ni(x, u) \mapsto f(x, u) \in \mathbb{R}
$$

satisfaz as condições de Carathéodory se

- $u \mapsto f(x, u)$ é contínua para quase todo $x \in \Omega$;

- $x \mapsto f(x, u)$ é mensurável para todo $u$.

Definição 1.0.17. Dada uma função $f$ satisfazendo as condições de Carathéodory e uma função $u: \Omega \rightarrow \mathbb{R}^{m}$, podemos definir uma outra função dada pela seguinte composição,

$$
\mathcal{F}(u)(x):=f(x, u(x))
$$

O operador composição $\mathcal{F}$ é chamado operador de Nemytskii.

A seguir exibiremos um teorema sobre a continuidade e limitação deste operador indo de $L^{p}$ em $L^{q}$.

Teorema 1.0.18. Sejam $\Omega \subset \mathbb{R}^{n}$ um dominio e

$$
\Omega \times \mathbb{R}^{m} \ni(x, u) \mapsto f(x, u) \in \mathbb{R}
$$


uma função satisfazendo as condições de Carathéodory. Mais ainda, suponha que $p \in$ $(1, \infty)$ e $g \in L^{q}(\Omega)$, onde $\frac{1}{p}+\frac{1}{q}=1$ tal que

$$
|f(x, u)| \leq c|u|^{p-1}+g(x)
$$

Então o operador de Nemytskii $\mathcal{F}$ é limitado, isto é, leva limitados em limitados, e é continuo de $L^{p}(\Omega)$ em $L^{q}(\Omega)$. 


\section{Teoria de Atratores}

\subsection{Introdução}

Neste capítulo exibiremos alguns conceitos e resultados relacionados a teoria de sistemas dinâmicos autônomos e não autônomos. Na teoria de sistemas dinâmicos não autônomos faremos duas abordagens distintas da dinâmica assintótica, especificamente, atratores pullback e atratores uniformes.

Os conceitos e resultados a seguir podem ser encontrados nos seguintes trabalhos $[10,14,45,26,33]$.

\section{$2.2 \quad$ Atratores para semigrupos}

Considere $X$ um espaço métrico munido da métrica $d: X \times X \rightarrow \mathbb{R}$ e denote $\mathbb{R}^{+}=\{t \in \mathbb{R} ; t \geq 0\}$.

Definição 2.2.1. Um semigrupo em $X$ é uma família $\left\{T(t) ; t \in \mathbb{R}^{+}\right\}$de aplicações contínuas de $X$ em $X$ tal que:

(i) $T(0)=I$, sendo $I$ o operador identidade de $X$;

(ii) $T(t+s)=T(t) \circ T(s)$, para todos $t, s \in \mathbb{R}^{+}$;

(iii) A aplicação $(t, x) \longmapsto T(t) x$ é contínua de $\mathbb{R}^{+} \times X$ em $X$.

Observação 2.2.2. Dizemos que o par $(X, T(t))$ é um sistema dinâmico, definido pelo semigrupo $\left\{T(t) ; t \in \mathbb{R}^{+}\right\}$.

Contextualizado pela definição de semigrupo, exibiremos alguns conceitos necessários para definir o atrator global de um sistema dinâmico. 
Definição 2.2.3. Um conjunto $A \subset X$ é dito invariante pelo semigrupo $T(\cdot)$ quando $T(t) A=A$, para todo $t \geq 0$.

Para o estudo do comportamento das soluções a longo prazo, uma ferramenta útil é a semidistância de Hausdorff, que nos permitirá "medir" a distância entre dois subconjuntos e nos dará uma noção de atração.

Definição 2.2.4. Sejam $A \subset X e B \subset X$ subconjuntos não vazios, definimos a semidistância de Hausdorff de $A$ até $B$ por

$$
\operatorname{dist}_{X}(A, B)=\sup _{a \in A} \inf _{b \in B} d(a, b)=\sup _{a \in A} d(a, B) .
$$

Definição 2.2.5. Dizemos que um conjunto $A \subset X$ atrai um conjunto $B \subset X$ pela ação do semigrupo $T(\cdot)$, quando

$$
\lim _{t \rightarrow \infty} \operatorname{dist}_{X}(T(t) B, A)=0,
$$

ou equivalentemente, se para todo $\varepsilon>0$ existir $\tau=\tau(\varepsilon, B)$ tal que

$$
T(t) B \subset \mathcal{O}_{\varepsilon}(A), \text { para todo } t \geq \tau,
$$

onde $\mathcal{O}_{\varepsilon}(A)$ é a $\varepsilon$-vizinhança de $A$, isto é, $\mathcal{O}_{\varepsilon}(A)=\{x \in X ; d(x, A)<\varepsilon\}$.

Sob essas considerações podemos definir a noção de atrator global.

Definição 2.2.6. Um subconjunto $\mathcal{A}$ de $X$ é dito ser atrator global para o semigrupo $T(\cdot)$, quando:

(i) $\mathcal{A}$ é compacto;

(ii) $\mathcal{A}$ é invariante;

(iii) $\mathcal{A}$ atrai cada um dos limitados de $X$ por meio do semigrupo $T(\cdot)$.

Afim de exibir um critério para existência de atratores globais os seguintes conceitos são de caráter fundamental.

Definição 2.2.7. Sejam $B \subset X$ e $D \subset X$. Dizemos que $D$ absorve o conjunto $B$ pela ação de $T(\cdot)$ quando existe $\tau=\tau(B)$ de modo que

$$
T(t) B \subset D, \text { para todo } t \geq \tau \text {. }
$$

Mais ainda, o semigrupo $T(\cdot)$ é dito ser dissipativo quando existe um conjunto limitado $D \subset X$ que absorve cada um dos limitados de $X$ pela ação de $T(\cdot)$. 
Definição 2.2.8. Um semigrupo $\left\{T(t) ; t \in \mathbb{R}^{+}\right\}$é dito ser assintóticamente compacto, quando para qualquer sequência $\left\{t_{n}\right\}_{n \in \mathbb{N}}$ com $_{n} \rightarrow \infty$ e qualquer sequência $\left\{x_{n}\right\}_{n \in \mathbb{N}}$ limitada em $X$, a sequência $\left\{T\left(t_{n}\right) x_{n}\right\}_{n \in \mathbb{N}}$ admite uma subsequência convergente.

A seguir, introduziremos o importante conceito de conjunto $\omega$-limite, que têm um papel fundamental no desenvolvimento da teoria de atratores globais.

Definição 2.2.9. Seja B um subconjunto de $X$. Definimos o seu conjunto $\omega$-limite como sendo:

$$
\omega(B)=\bigcap_{t \geq 0} \overline{\bigcup_{s \geq t} T(s) B},
$$

ou equivalentemente,

$$
\begin{aligned}
\omega(B)=\left\{x \in X ; \text { existem sequência }\left\{t_{n}\right\} \text { com } t_{n}\right. & \rightarrow \infty \\
e & \left.\left\{x_{n}\right\} \subset B \text { tal que } x=\lim _{n \rightarrow \infty} T\left(t_{n}\right) x_{n}\right\} .
\end{aligned}
$$

Para finalizar essa seção, vamos enunciar uma condição necessária e suficiente para existência do atrator global de um sistema dinâmico $(X, T(t))$.

Teorema 2.2.10. Um semigrupo $\left\{T(t) ; t \in \mathbb{R}^{+}\right\}$admite um atrator global $\mathcal{A}$ se, e somente se, é dissipativo e assintóticamente compacto. Em caso afirmativo, o atrator global é dado por

$$
\mathcal{A}=\bigcup_{B \in \mathcal{B}} \omega(B)
$$

onde $\mathcal{B}$ é a coleção de todos os limitados de $X$.

\subsection{Atrator pullback}

Nesta seção iremos exibir uma visão pullback de um sistema dinâmico não autônomo. Começamos com a definição de um dos principais objetos da teoria não autônoma, o chamado processo de evolução. Os conceitos e resultados exibidos aqui foram baseados nos trabalhos $[10,14,26]$.

Definição 2.3.1. Um processo de evolução sobre um espaço métrico X é uma família a dois parâmetros $\{U(t, \tau) ; t \geq \tau \in \mathbb{R}\}$ de aplicações de $X$ em $X$ tais que:

(i) $U(t, t)=I$, sendo $I$ o operador identidade de $X$, para todo $t \in \mathbb{R}$; 
(ii) $U(t, s) \circ U(s, \tau)=U(t, \tau)$, sempre que $t \geq s \geq \tau$ em $\mathbb{R}$.

Definição 2.3.2. Um processo de evolução $\{U(t, \tau)\}_{t \geq \tau}$ é chamado de fechado se, sempre que uma sequência $x_{n} \rightarrow x$ em $X$ e $U(t, \tau) x_{n} \rightarrow y$ para algum $y \in X$, então $U(t, \tau) x=y$, para quaisquer $t \geq \tau$.

Observação 2.3.3. Em geral, se uma equação de evolução não-autônoma tem uma única solução que depende continuamente dos dados iniciais, então esse problema gera um processo de evolução contínuo, no sentido de que $U(t, \tau): X \rightarrow X$ é uma aplicação contínua para todos $t \geq \tau$. Claramente um processo contínuo é também um processo de evolução fechado.

No que segue, denotaremos por $\widehat{D}$ uma família de subconjuntos de $X$, indexadas em $t \in \mathbb{R}$, isto é:

$$
\widehat{D}=\{D(t) \subset X ; t \in \mathbb{R}\}
$$

A seguir, vamos definir o universo de famílias a serem atraídas pelo atrator.

Definição 2.3.4. Um universo do espaço métrico $X$ é uma classe $\mathcal{D}$ formada de elementos $\widehat{D}=\{D(t)\}_{t \in \mathbb{R}}$ tal que cada seção $D(t)$ é um subconjunto não vazio de $X$, para todo $t \in \mathbb{R}$. Mais ainda, dizemos que um universo $\mathcal{D}$ é fechado por inclusão se, dados $\widehat{D} \in \mathcal{D}$ e $\widehat{C}$ com $C(t) \subset D(t)$, para todo $t \in \mathbb{R}$, então $\widehat{C} \in \mathcal{D}$.

Definição 2.3.5. Uma família $\widehat{D}$ é dita invariante pelo processo de evolução $U(\cdot, \cdot)$ se $U(t, \tau) D(\tau)=D(t)$, para todo $t \geq \tau$.

Assim podemos definir a noção de atração no sentido pullback.

Definição 2.3.6. Sejam $U(\cdot, \cdot)$ um processo em um espaço métrico $X$ e $\mathcal{D}$ um universo em $X$.

(a) Dizemos que uma família $\widehat{B} \mathcal{D}$-atrai no sentido pullback se, para cada $\widehat{D} \in \mathcal{D}$ tem-se que

$$
\lim _{\tau \rightarrow-\infty} \operatorname{dist}_{X}(U(t, \tau) D(\tau), B(t))=0
$$

para todo $t \in \mathbb{R}$. 
(b) Uma família $\widehat{B}$ de conjuntos não vazios de $X$ é chamada de $\mathcal{D}$-absorvente no sentido pullback para o processo $U(\cdot, \cdot)$ se dados $t \in \mathbb{R}$ e $\widehat{D} \in \mathcal{D}$, existe $T(t, \widehat{D}) \leq t$ tal que

$$
U(t, \tau) D(\tau) \subset B(t)
$$

para todo $\tau \leq T(t, \widehat{D})$.

Salvo essas considerações, segue a seguinte definição.

Definição 2.3.7. Uma família $\widehat{\mathcal{A}}$ é um $\mathcal{D}$-atrator pullback para o processo de evolução $\{U(t, \tau) ; t \geq \tau \in \mathbb{R}\}$ se,

(i) para todo $t \in \mathbb{R}, \mathcal{A}(t)$ é um subconjunto compacto e não vazio de $X$;

(ii) a família $\widehat{\mathcal{A}} \mathcal{D}$-atrai no sentido pullback;

(iii) a família $\widehat{\mathcal{A}}$ é invariante.

Mais ainda, um $\mathcal{D}$-atrator pullback $\widehat{\mathcal{A}}$ é dito ser minimal se, dada uma família $\widehat{C}$ de subconjuntos fechados e não vazios de $X$ que satisfaz a propriedade (ii), então $\mathcal{A}(t) \subset C(t)$ para todo $t \in \mathbb{R}$.

Análogo à teoria de sistemas dinâmicos autônomos, a compacidade assintótica no sentido pullback será fundamental na dinâmica não autônoma.

Definição 2.3.8. Dizemos que um processo de evolução $U(\cdot, \cdot)$ é $\mathcal{D}$-assintóticamente compacto no sentido pullback se, dados qualquer $t \in \mathbb{R}$, qualquer $\widehat{D} \in \mathcal{D}$ e, quaisquer sequências $\left\{\tau_{k}\right\} \subset(-\infty, t]$ e $\left\{x_{k}\right\} \subset X$ satisfazendo $\tau_{k} \rightarrow-\infty$ quando $k \rightarrow \infty$ e $x_{k} \in$ $D\left(\tau_{k}\right)$ para todo $k$, então a sequência $\left\{U\left(t, \tau_{k}\right) x_{k}\right\}$ é relativamente compacta em $X$.

Com as definições acima, obtemos o principal resultado dessa seção cuja demonstração pode ser encontrada nos trabalhos [10, 26].

Teorema 2.3.9. Seja $\{U(t, \tau) ; t \geq \tau \in \mathbb{R}\}$ um processo de evolução sobre um espaço métrico $X$ e considere $\mathcal{D}$ um universo em $X$ fixado. Suponha que o processo $\{U(t, \tau)\}_{t \geq \tau}$ é $\mathcal{D}$-assintóticamente compacto no sentido pullback e admite um família $\widehat{B}_{0}=\left\{B_{0}(t)\right\}_{t \in \mathbb{R}}$ $\mathcal{D}$-absorvente no sentido pullback. Então, a família $\widehat{\mathcal{A}}_{\mathcal{D}}=\left\{A_{0}(t)\right\}_{t \in \mathbb{R}}$ definida por

$$
A_{0}(t)=\overline{\bigcup_{\widehat{D} \in \mathcal{D}} \Lambda(\widehat{D}, t)},
$$


é um $\mathcal{D}$-atrator pullback minimal para o processo $U(\cdot, \cdot)$. Aqui $\Lambda$ denota o $\omega$-limite pullback, isto é,

$$
\Lambda(\widehat{D}, t)=\bigcap_{s \leq t \leq s} U(t, \tau) D(\tau) .
$$

Além disso, se $\widehat{B}_{0} \in \mathcal{D}$, então

$$
A_{0}(t)=\Lambda\left(\widehat{B}_{0}, t\right) \subset \overline{B_{0}(t)}
$$

Mais ainda, se o universo $\mathcal{D}$ é fechado por inclusão e $\widehat{B}_{0} \in \mathcal{D}$, então $\widehat{\mathcal{A}}_{\mathcal{D}} \in \mathcal{D}$.

\subsubsection{Critério para compacidade assintótica}

A seguir vamos apresentar uma condição suficiente para compacidade assintótica no sentido pullback de um processo de evolução (segundo a Definição 2.3.8). No caso autônomo tal critério foi proposto por Chueshov e Lasiecka no trabalho [17]. Algumas versões não autônomas podem ser encontradas nos seguintes trabalhos [33, 41, 42].

Definição 2.3.10. Seja $X$ um espaço métrico. Dizemos que uma função $\Psi: X \times X \rightarrow \mathbb{R}$ é contrativa sobre um subconjunto limitado $B$ de $X$ se, para qualquer sequência $\left\{x_{n}\right\}$ de $B$ existe uma subsequência $\left\{x_{n_{k}}\right\}$ tal que

$$
\lim _{k \rightarrow \infty} \lim _{l \rightarrow \infty} \Psi\left(x_{n_{k}}, x_{n_{l}}\right)=0
$$

Temos então o seguinte critério da compacidade assintótica no sentido pullback [33], cuja prova é apresentada para a conveniência do leitor.

Teorema 2.3.11. Sejam $X$ um espaço de Banach e $U(\cdot, \cdot)$ um processo que admite uma família de subconjuntos limitados de $X$ que é $\mathcal{D}$-absorvente no sentido pullback, digamos $\widehat{B}_{0}=\left\{B_{0}(t)\right\}_{t \in \mathbb{R}}$. Suponha que para qualquer $t \in \mathbb{R}$ e qualquer $\varepsilon>0$ existe $\tau_{\varepsilon} \leq t$ e uma função $\Psi_{\varepsilon}$ contrativa sobre $B_{0}\left(\tau_{\varepsilon}\right)$, tal que

$$
\left\|U\left(t, \tau_{\varepsilon}\right) x-U\left(t, \tau_{\varepsilon}\right) y\right\|_{X} \leq \varepsilon+\Psi_{\varepsilon}(x, y) \text {, para quaisquer } x, y \in B_{0}\left(\tau_{\varepsilon}\right) .
$$

Então o processo $\{U(t, \tau) ; t \geq \tau \in \mathbb{R}\}$ é $\mathcal{D}$-assintoticamente compacto no sentido pullback.

Demonstração. Pela Definição 2.3.8, dados qualquer $t \in \mathbb{R}$, qualquer $\widehat{D} \in \mathcal{D}$ e, quaisquer sequências $\left\{\tau_{k}\right\}_{k \in \mathbb{N}} \subset(-\infty, t]$ com $\tau_{k} \rightarrow-\infty$ e $\left\{x_{k}\right\}_{k \in \mathbb{N}} \subset X$ tal que $x_{k} \in D\left(\tau_{k}\right)$ para 
todo $k \in \mathbb{N}$, devemos provar que a sequência da forma $\left\{U\left(t, \tau_{k}\right) x_{k}\right\}$ tem uma subsequência convergente. Com efeito:

Seja $\left\{\varepsilon_{n}\right\}_{n \in \mathbb{N}}$ uma sequência real tal que $\varepsilon_{n} \rightarrow 0$ e $\varepsilon_{n}>0$ para todo $n \in \mathbb{N}$. Então, pela hipótese, existem um $\tau_{\varepsilon_{1}} \leq t$ e uma função contrativa $\Psi_{\varepsilon_{1}}$ sobre $B_{0}\left(\tau_{\varepsilon_{1}}\right)$ tais que

$$
\left\|U\left(t, \tau_{\varepsilon_{1}}\right) x-U\left(t, \tau_{\varepsilon_{1}}\right) y\right\|_{X} \leq \frac{\varepsilon_{1}}{2}+\Psi_{\varepsilon_{1}}(x, y)
$$

para todos $x, y \in B_{0}\left(\tau_{\varepsilon_{1}}\right)$.

Observe que, como $\tau_{k} \rightarrow-\infty$ então existe um $k_{0} \in \mathbb{N}$ tal que

$$
\tau_{k} \leq \tau_{k_{0}} \leq \tau_{\varepsilon_{1}}, \text { para todo } k \geq k_{0},
$$

e

$$
U\left(\tau_{\varepsilon_{1}}, \tau_{k}\right) D\left(\tau_{k}\right) \subset B_{0}\left(\tau_{\varepsilon_{1}}\right), \text { para todo } k \geq k_{0}
$$

Passando para uma subsequência se necessário, podemos assumir que

$$
y_{k}^{\tau_{\varepsilon_{1}}}=U\left(\tau_{\varepsilon_{1}}, \tau_{k}\right) x_{k} \in B_{0}\left(\tau_{\varepsilon_{1}}\right)
$$

para todo $k \in \mathbb{N}$. Usando o fato de que $\Psi_{\varepsilon_{1}}$ é contrativa sobre $B_{0}\left(\tau_{\varepsilon_{1}}\right)$, concluímos que existem subsequência $\left\{x_{k}^{1}\right\}$ de $\left\{x_{k}\right\}$ e subsequência $\left\{\tau_{k}^{1}\right\}$ de $\left\{\tau_{k}\right\}$ tais que

$$
\Psi_{\varepsilon_{1}}\left(y_{k}^{\tau_{\varepsilon_{1}}, 1}, y_{l}^{\tau_{\varepsilon_{1}}, 1}\right)<\frac{\varepsilon_{1}}{2}
$$

para todos $k, l \in \mathbb{N}$. Assim, para todos $k, l \in \mathbb{N}$,

$$
\left\|U\left(t, \tau_{k}^{1}\right) x_{k}^{1}-U\left(t, \tau_{l}^{1}\right) x_{l}^{1}\right\|_{X}=\left\|U\left(t, \tau_{\varepsilon_{1}}\right) y_{k}^{\tau_{\varepsilon_{1}}, 1}-U\left(t, \tau_{\varepsilon_{1}}\right) y_{l}^{\tau_{\varepsilon_{1}}, 1}\right\|_{X}<\varepsilon_{1} .
$$

Agora, procedendo analogamente, existem $\tau_{\varepsilon_{2}} \leq t$ e uma função contrativa $\Psi_{\varepsilon_{2}}$ sobre $B_{0}\left(\tau_{\varepsilon_{2}}\right)$ tais que

$$
\left\|U\left(t, \tau_{\varepsilon_{2}}\right) x-U\left(t, \tau_{\varepsilon_{2}}\right) y\right\|_{X} \leq \frac{\varepsilon_{2}}{2}+\Psi_{\varepsilon_{2}}(x, y),
$$

para todos $x, y \in B_{0}\left(\tau_{\varepsilon_{2}}\right)$.

Observe que $\tau_{k}^{1} \rightarrow-\infty$, então existe um $k_{0} \in \mathbb{N}$ tal que

$$
\begin{gathered}
\tau_{k}^{1} \leq \tau_{k_{0}}^{1} \leq \tau_{\varepsilon_{2}}, \text { para todo } k \geq k_{0}, \\
U\left(\tau_{\varepsilon_{2}}, \tau_{k}^{1}\right) D\left(\tau_{k}^{1}\right) \subset B_{0}\left(\tau_{\varepsilon_{2}}\right), \text { para todo } k \geq k_{0} .
\end{gathered}
$$


Passando para uma subsequência se necessário, podemos assumir que

$$
y_{k}^{\tau_{\varepsilon_{2}}}=U\left(\tau_{\varepsilon_{2}}, \tau_{k}^{1}\right) x_{k}^{1} \in B_{0}\left(\tau_{\varepsilon_{2}}\right), \text { para todo } k \in \mathbb{N} .
$$

Pelo fato de que $\Psi_{\varepsilon_{2}}$ é contrativa sobre $B_{0}\left(\tau_{\varepsilon_{2}}\right)$, devem existir subsequências $\left\{x_{k}^{2}\right\}$ de $\left\{x_{k}^{1}\right\}$ e $\left\{\tau_{k}^{2}\right\}$ de $\left\{\tau_{k}^{1}\right\}$ tais que

$$
\Psi_{\varepsilon_{2}}\left(y_{k}^{\tau_{\varepsilon_{2}}, 2}, y_{l}^{\tau_{\varepsilon_{2}}, 2}\right)<\frac{\varepsilon_{2}}{2}, \text { para todos } k, l \in \mathbb{N},
$$

onde $y_{k}^{\tau_{\varepsilon_{2}}, 2}=U\left(\tau_{\varepsilon_{2}}, \tau_{k}^{2}\right) x_{k}^{2}$ é uma subsequência de $y_{k}^{\tau_{\varepsilon_{2}}}$. Logo, para todos $k, l \in \mathbb{N}$,

$$
\left\|U\left(t, \tau_{k}^{2}\right) x_{k}^{2}-U\left(t, \tau_{l}^{2}\right) x_{l}^{2}\right\|_{X}=\left\|U\left(t, \tau_{\varepsilon_{2}}\right) y_{k}^{\tau_{\varepsilon_{2}}, 2}-U\left(t, \tau_{\varepsilon_{2}}\right) y_{l}^{\tau_{\varepsilon_{2}}, 2}\right\|_{X}<\varepsilon_{2}
$$

Finalmente, repetindo esse argumento $n$ vezes, obtemos subsequência $\left\{x_{k}^{n}\right\}$ de $\left\{x_{k}^{n-1}\right\}$ e $\left\{\tau_{k}^{n}\right\}$ de $\left\{\tau_{k}^{n-1}\right\}$ de modo que, para todos $k, l \in \mathbb{N}$,

$$
\left\|U\left(t, \tau_{k}^{n}\right) x_{k}^{n}-U\left(t, \tau_{l}^{n}\right) x_{l}^{n}\right\|_{X}=\left\|U\left(t, \tau_{\varepsilon_{n}}\right) y_{k}^{\tau_{\varepsilon_{n}}, n}-U\left(t, \tau_{\varepsilon_{n}}\right) y_{l}^{\tau_{\varepsilon_{n}}, n}\right\|_{X}<\varepsilon_{n} .
$$

Como $\varepsilon_{n} \rightarrow 0$, obtemos que a subsequência diagonal $\left\{U\left(t, \tau_{k}^{k}\right) x_{k}^{k}\right\}$ de $\left\{U\left(t, \tau_{k}\right) x_{k}\right\}$ é uma sequência de Cauchy no espaço de Banach $X$, para tal, basta notar que:

$$
\left\|U\left(t, \tau_{k}^{k}\right) x_{k}^{k}-U\left(t, \tau_{l}^{l}\right) x_{l}^{l}\right\|_{X}<\varepsilon_{l} \text {, desde que } k \geq l
$$

Isto conclui a demonstração.

\subsubsection{Semicontinuidade superior de atratores}

Nesta seção consideraremos o universo $\mathcal{D}$ formado por famílias constantes e limitadas, isto é, formado por famílias $\widehat{D}=\{D(t)\}$ onde $D(t)=D$ para todo $t \in \mathbb{R}$, sendo $D$ um subconjunto limitado de $X$. Os próximos resultados relacionam os atratores pullback de sistemas dinâmicos não autônomos e os atratores globais para sistemas dinâmicos autônomos. Os conceitos e resultados dessa seção podem ser encontrados no livro [10].

O primeiro resultado confirma que a noção de atrator pullback extende, de maneira natural o conceito de atrator global sobre certas condições.

Lema 2.3.12. Sejam $T(t): X \rightarrow X$ um semigrupo e $S_{T}(t, \tau): X \rightarrow X$ o processo de evolução induzido pelo semigrupo, isto é, $S_{T}(t, \tau)=T(t-\tau)$ para $t \geq \tau$. Então $T(t)$ tem um atrator global $\mathcal{A}$ se, e somente se $S_{T}(t, \tau)$ tem um atrator pullback $\widehat{\mathcal{A}}=\{\mathcal{A}(t)\}$. Em caso afirmativo a família $\widehat{\mathcal{A}}$ é constante, onde $\mathcal{A}(t)=\mathcal{A}$ para todo $t \in \mathbb{R}$. 
Agora vamos comparar atratores pullback de dois processos de evolução próximos, em algum sentido. Antes iremos definir uma noção de continuidade de um família de atratores pullback.

Definição 2.3.13. Dizemos que uma família de atratores pullback $\left\{\widehat{\mathcal{A}}_{\varepsilon}\right\}_{\varepsilon \in[0,1)}$ é semicontínua superiormente em $\varepsilon_{0} \in[0,1)$ sempre que

$$
\lim _{\varepsilon \rightarrow \varepsilon_{0}} \operatorname{dist}\left(\mathcal{A}_{\varepsilon}(t), \mathcal{A}(t)\right)=0
$$

para todo $t \in \mathbb{R}$.

Proposição 2.3.14. Suponha que $S_{\varepsilon}(t, \tau): X \rightarrow X$ é uma família de processos indexados por $\varepsilon \in[0,1)$ tal que

(i) $S_{\varepsilon}(t, \tau)$ tem um atrator pullback minimal $\widehat{\mathcal{A}}_{\varepsilon}$ para todo $\varepsilon \in[0,1)$;

(ii) Para todo $t \in \mathbb{R}$, todo $T \geq 0$ e todo conjunto limitado $D \subset X$,

$$
\sup _{\tau \in[0, T], u_{0} \in D} d\left(S_{\varepsilon}(t+\tau, t) u_{0}, S_{0}(t+\tau, t) u_{0}\right) \rightarrow 0, \quad \text { quando } \quad \varepsilon \rightarrow 0 .
$$

(iii) Existe $\delta>0$ e $t_{0} \in \mathbb{R}$ tal que

$$
\bigcup_{\varepsilon \in(0, \delta)} \bigcup_{s \leq t_{0}} \mathcal{A}_{\varepsilon}(s)
$$

é limitado.

Então a família de processos $S_{\varepsilon}(t, \tau): X \rightarrow X$ é semicontínua superiormente em $\varepsilon=0$.

\subsection{Atrator uniforme}

Ainda no contexto de sistemas dinâmicos não autônomos, nesta seção, iremos exibir um outro ponto de vista dos atratores para sistemas dinâmicos não autônomos. Aqui a ideia será reduzir o sistema não autônomo em um autônomo, sobre apropriados espaços de fase. Os conceitos e resultados foram baseados nos trabalhos [14, 45].

Começamos com a definição de algumas classes admissíveis de forças externas que dependem do tempo e que serão usadas no decorrer desta tese. Nesta seção vamos estudar as relações entre tais classes. 
Seja $V$ um espaço de Banach reflexivo. Lembrando que $L_{l o c}^{p}(\mathbb{R} ; V)$ é o espaço de todas as funções $g(s), s \in \mathbb{R}$, com valores em $V$ e $p$-localmente integrável, $p \geq 1$, isto é,

$$
\int_{t_{1}}^{t_{2}}\|g(s)\|_{V}^{p} d s<\infty
$$

para todo intervalo $\left[t_{1}, t_{2}\right] \subset \mathbb{R}$. Denote $L_{b}^{p}(\mathbb{R} ; V)$ o espaço de todas as funções $g$ de $L_{l o c}^{p}(\mathbb{R} ; V)$ tal que

$$
\sup _{t \in \mathbb{R}} \int_{t}^{t+1}\|g(s)\|_{V}^{p} d s<\infty
$$

As funções pertencentes ao espaço $L_{b}^{p}(\mathbb{R} ; V)$ são chamadas de funções de translação limitada. Neste espaço, definimos a seguinte norma

$$
\|g\|_{L_{b}^{p}(\mathbb{R} ; V)}=\left(\sup _{t \in \mathbb{R}} \int_{t}^{t+1}\|g(s)\|_{V}^{p} d s\right)^{\frac{1}{p}} .
$$

A seguir exibiremos um lema técnico que compara as normas dos espaços introduzidos acima, cuja demonstração pode ser encontrada no Lema II.1.3 do livro [14].

Lema 2.4.1. Seja $\sigma>0$ qualquer constante positiva e considere $g \in L_{\text {loc }}^{p}(\mathbb{R} ; V)$. Então

$$
\int_{\tau}^{t} e^{-\sigma(t-s)}\|g(s)\|_{V}^{p} d s \leq \frac{1}{1-e^{-\sigma}} \sup _{t \in \mathbb{R}} \int_{t}^{t+1}\|g(s)\|_{V}^{p} d s
$$

para quaisquer $t \geq \tau$. Melhor ainda, se $g \in L_{b}^{p}(\mathbb{R} ; V)$ então

$$
\int_{\tau}^{t} e^{-\sigma(t-s)}\|g(s)\|_{V}^{p} d s \leq \frac{1}{1-e^{-\sigma}}\|g(s)\|_{L_{b}^{p}(\mathbb{R} ; V)}^{p} .
$$

\subsubsection{Forças externas de translação compacta}

Note que o espaço $L_{b}^{p}(\mathbb{R} ; V)$ é invariante com respeito as translações no tempo, ou seja, considere $g \in L_{b}^{p}(\mathbb{R} ; V)$ e defina

$$
(T(s) g)(t)=g(t+s) .
$$

Então,

$$
T(s) g \in L_{b}^{p}(\mathbb{R} ; V)
$$

para todo $s \in \mathbb{R}$. Portanto podemos definir a orbita de $g \in L_{b}^{p}(\mathbb{R} ; V)$ sobre o grupo de translações $T$ da seguinte forma

$$
\mathcal{O}(g)=\{T(s) g ; s \in \mathbb{R}\} \subset L_{b}^{p}(\mathbb{R} ; V) .
$$

Assim, dizemos que uma função $g \in L_{b}^{p}(\mathbb{R} ; V)$ é de translação compacta se a orbita $\mathcal{O}(g)$ é pré-compacta no espaço de Frechet $L_{l o c}^{p}(\mathbb{R} ; V)$. 
Proposição 2.4.2. Uma função $g \in L_{b}^{p}(\mathbb{R} ; V)$ é de translação compacta se, e somente se

1) A função g tem uma continuidade do módulo $L^{p}$, ou seja, existe uma função monótona $w: \mathbb{R}_{+} \rightarrow \mathbb{R}_{+}$tal que $\lim _{z \rightarrow 0} w(z)=0 e$

$$
\sup _{t \in \mathbb{R}} \int_{t}^{t+1}\|g(s+\tau)-g(s)\|_{V}^{p} d s \leq w(\tau)
$$

2) Considere $g_{h}(t)=\frac{1}{h} \int_{t}^{t+h} g(s) d s$. Então a imagem de $g_{h}$ é pré-compacta em $V$, para cada $h \in(0, \infty)$, isto é, o conjunto

$$
\left\{g_{h}(t) ; t \in \mathbb{R}\right\} \subset V,
$$

é pré-compacto em $V$, para cada $h \in(0, \infty)$.

\subsubsection{Forças externas regulares no espaço}

A grosso modo, as forças externas de translação compacta são aquelas que tem certo tipo de regularidade no espaço e no tempo são regulares, mais especificamente, podem ser aproximadas por funções suaves no espaço e no tempo. Nas próximas definições, apresentaremos novas classes de forças externas que são apenas regulares no espaço ou no tempo. Como veremos a seguir tal regularidade parcial é suficiente para a compacidade assintótica de uma grande classe de problemas não autônomos.

Definição 2.4.3. Uma função $g \in L_{b}^{p}(\mathbb{R} ; V)$ é regular no espaço se para todo $\varepsilon>0$ existe um subespaço de dimensão finita $V_{\varepsilon} \subset V$ e uma função $g_{\varepsilon} \in L_{b}^{p}(\mathbb{R} ; V)$ tal que

$$
\left\|g-g_{\varepsilon}\right\|_{L_{b}^{p}(\mathbb{R} ; V)} \leq \varepsilon .
$$

Em outras palavras, uma função $g \in L_{b}^{p}(\mathbb{R} ; V)$ é dita ser regular no espaço se pode ser aproximada por funções com imagem de dimensão finita.

A próxima proposição mostra um típico exemplo para funções regulares no espaço.

Proposição 2.4.4. Sejam $W \stackrel{c}{\hookrightarrow} V$ e $g \in L_{b}^{p}(\mathbb{R} ; W)$. Então a função $g$ é regular no espaço como uma função de $L_{b}^{p}(\mathbb{R} ; V)$.

Observação 2.4.5. Nas aplicações geralmente $V=L^{p}(\Omega)$ ou $V=W^{s, p}(\Omega)$. Neste caso, pela perturbação arbitrária e suficientemente pequena do espaço de dimensão finita $V_{\varepsilon}$, 
envolvido na Definição 2.4.3, podemos escolher $V_{\varepsilon}$ regular, por exemplo $V_{\varepsilon}=C^{\infty}(\Omega)$. Assim, sem perda de generalidade, podemos assumir que a aproximação $g_{\varepsilon}$ é suave no espaço. Esta simples observação é crucial para prova da compacidade assintótica no caso de EDPs com forças externas regulares no espaço.

\subsubsection{Forças externas regulares no tempo}

Começamos com a seguinte definição.

Definição 2.4.6. Uma função $g \in L_{b}^{p}(\mathbb{R} ; V)$ é regular no tempo se para todo $\varepsilon>0$ existe uma função $g_{\varepsilon} \in C_{b}^{k}(\mathbb{R} ; V)$, para qualquer $k>0$, tal que

$$
\left\|g-g_{\varepsilon}\right\|_{L_{b}^{p}(\mathbb{R} ; V)} \leq \varepsilon
$$

Em outras palavras, a função $g \in L_{b}^{p}(\mathbb{R} ; V)$ pode ser aproximada em $L_{b}^{p}(\mathbb{R} ; V)$ por funções suaves no tempo.

Observação 2.4.7. A hipótese $g_{\varepsilon} \in C_{b}^{k}(\mathbb{R} ; V)$ as vezes não é conveniente pois os espaços $C^{k}$ não são reflexivos e não temos a compacidade fraca da bola unitária. Por esta razão, ao invés de $C^{k}$, vamos usar a hipótese de que $g_{\varepsilon}$ pertence ao seguinte espaço $H_{b}^{k}(\mathbb{R} ; V)$.

A próxima proposição estabelece uma importante caracterização das forças externas regulares no tempo.

Proposição 2.4.8. Uma função $g \in L_{b}^{p}(\mathbb{R} ; V)$ é regular no tempo se, e somente se possui uma continuidade do módulo em $L_{b}^{p}$, isto é, existe uma função monótona decrescente $w: \mathbb{R}_{+} \rightarrow \mathbb{R}_{+}$tal que $\lim _{z \rightarrow 0} w(z)=0 e$

$$
\sup _{t \in \mathbb{R}} \int_{t}^{t+1}\|g(s+\tau)-g(s)\|_{V}^{p} d s \leq w(z) .
$$

Uma pergunta pertinente a se fazer é: Qual a relação entre as forças externas regulares no espaço e regulares no tempo com as funções de translação compacta?

A resposta vem com o seguinte resultado.

Teorema 2.4.9. Uma função $g \in L_{b}^{p}(\mathbb{R} ; V)$ é regular no espaço e regular no tempo se, e somente se $g$ é de translação compacta.

Observação 2.4.10. Como podemos ver, as duas classes de forças externas introduzidas, regulares no espaço e regulares no tempo, se interceptam exatamente na classe das funções de translação compacta. 


\subsubsection{Forças externas: Normal, fracamente normal e fortemente normal}

As forças externas normais foram a primeira grande classe de funções que não são de translação compacta porém ainda é possível mostrar a compacidade assintótica forte em certas EDPs.

Definição 2.4.11. Seja $g \in L_{b}^{p}(\mathbb{R} ; V)$. Dizemos que g é normal se

$$
\sup _{t \in \mathbb{R}} \int_{t}^{t+\tau}\|g(s)\|_{V}^{p} d s \rightarrow 0
$$

quando $\tau \rightarrow 0$.

Observação 2.4.12. Um típico exemplo de função normal no espaço $L_{b}^{p}(\mathbb{R} ; V)$ é $g \in$ $L_{b}^{p+\varepsilon}(\mathbb{R} ; V)$ para algum $\varepsilon>0$. Embora existem mais exemplos complicados.

As aplicações da forças externas normais para equações parabólicas são baseadas no seguinte lema.

Lema 2.4.13. Seja $g \in L_{b}^{p}(\mathbb{R} ; V)$ normal. Então

$$
\sup _{t \in \mathbb{R}} \int_{t-1}^{t} e^{N(t-s)} g(s) d s \rightarrow 0
$$

quando $N \rightarrow \infty$.

Podemos introduzir mais uma classe de forças externas, que é mais fraca do que a classe das forças normais, para de alguma forma unificar as classes regulares no espaço, regulares no tempo e as forças externas normais (cf. [45]).

Definição 2.4.14. Seja $g \in L_{b}^{p}(\mathbb{R} ; V)$ uma força externa de translação limitada. Então, a força externa $g$ é fracamente normal se para qualquer $\varepsilon>0$ existe $\tau=\tau(\varepsilon)>0$, um espaço de dimensão finita $V_{\varepsilon} \subset V$ e uma função $g_{\varepsilon} \in L_{b}^{p}\left(\mathbb{R} ; V_{\varepsilon}\right)$ tais que

$$
\sup _{t \in \mathbb{R}} \int_{t}^{t+\tau}\left\|g(s)-g_{\varepsilon}(s)\right\|_{V}^{p} d s \leq \varepsilon .
$$

Lema 2.4.15. Seja $g \in L_{b}^{p}(\mathbb{R} ; V)$ fracamente normal. Então existe uma função monótona $w: \mathbb{R}_{+} \rightarrow \mathbb{R}_{+}$satisfazendo $\lim _{z \rightarrow 0} w(z)=0$ tal que, para todo $\varepsilon>0$,

$$
\limsup _{N \rightarrow \infty}\left[\sup _{t \in \mathbb{R}} \int_{t-1}^{t} e^{-N(t-s)}\left\|g(s)-g_{\varepsilon}(s)\right\|_{V}^{p} d s\right] \leq w(\varepsilon),
$$

onde $g_{\varepsilon}$ é como na Definição 2.4.14. 
A próxima proposição mostra que todas as classes de forças externas introduzidas até agora estão contidas na classe das forças externas fracamente normais.

Proposição 2.4.16. Considere uma força externa $g \in L_{b}^{2}(\mathbb{R} ; V)$ regular no espaço ou regular no tempo ou normal. Então g é fracamente normal.

Como podemos ver, a definição de força externa normal tem uma estrutura diferente da definição de força regular no espaço e de força regular no tempo, na realidade ainda não se sabe com definir as forças normais usando aproximações. Porém, exibiremos uma classe de forças externas ligeiramente mais restritiva e que está contida na classe de forças normais. Essas forças podem ser descritas por aproximações.

Definição 2.4.17. Uma função $g \in L_{b}^{p}(\mathbb{R} ; V)$ é fortemente normal se para qualquer $\varepsilon>0$ existe uma função $g_{\varepsilon} \in L^{\infty}(\mathbb{R} ; V)$ tal que

$$
\left\|g-g_{\varepsilon}\right\|_{L_{b}^{p}(\mathbb{R} ; V)} \leq \varepsilon
$$

Proposição 2.4.18. 1) Seja $g \in L_{b}^{p}(\mathbb{R} ; V)$ fortemente normal, então g é normal.

2) Seja $g \in L_{b}^{p+\varepsilon}(\mathbb{R} ; V)$ para algum $\varepsilon>0$, então g é fortemente normal em $L_{b}^{p}(\mathbb{R} ; V)$.

3) Seja $g \in L_{b}^{p}(\mathbb{R} ; V)$ regular no tempo, entao g é fortemente normal.

\subsubsection{Envoltória fraca para forças externas de translação limi- tada}

Nesta seção, iremos mostrar algumas ferramentas técnicas que vão permitir checar a compacidade assintótica em certas classes de forças externas até agora definidas. Primeiramente vamos definir a envoltória fraca de uma função de translação limitada $g \in L_{b}^{p}(\mathbb{R} ; V)$.

Observação 2.4.19. Ao contrário das funções de translação compacta, agora a orbita $\mathcal{O}(g)$ não é pré-compacta na topologia forte de $L_{\text {loc }}^{p}(\mathbb{R} ; V)$. Entretanto, como $L_{\text {loc }}^{p}(\mathbb{R} ; V)$ é reflexivo, a orbita $\mathcal{O}(g)$ é pré-compacta na topologia fraca de $L_{\text {loc }}^{p}(\mathbb{R} ; V)$. O espaço $L_{\text {loc }}^{p}(\mathbb{R} ; V)$ munido com a topologia fraca será denotado por $L_{l o c, w}^{p}(\mathbb{R} ; V)$. Lembrando que uma sequência $v_{n} \rightarrow v$ em $L_{l o c, w}^{p}(\mathbb{R} ; V)$, se para todo intervalo $\left[t_{1}, t_{2}\right]$ a sequência $\left.v_{n}\right|_{\left[t_{1}, t_{2}\right]}$ converge fraco para função $\left.v\right|_{\left[t_{1}, t_{2}\right]}$ em $L^{p}\left(t_{1}, t_{2} ; V\right)$. 
Definição 2.4.20. Seja $g \in L_{l o c}^{p}(\mathbb{R} ; V)$ uma função de translação limitada. Então a envoltória fraca $\mathcal{H}(g)$ é definida como o fecho da orbita $\mathcal{O}(g)$ no espaço $L_{\text {loc,w }}^{p}(\mathbb{R} ; V)$, isto $\dot{e}$,

$$
\mathcal{H}(g)=\overline{\mathcal{O}(g)} L_{l o c, w}^{p}(\mathbb{R} ; V)
$$

Observe que a ação do grupo de translações $T(\cdot)$ na envoltória fraca satisfaz a seguinte propriedade:

$$
T(s): \mathcal{H}(g) \rightarrow \mathcal{H}(g), s \in \mathbb{R} \text { e } T(s) \mathcal{H}(g)=\mathcal{H}(g)
$$

Esta simples observação é muito importante para reduzir o processo não autônomo, em um semigrupo.

Proposição 2.4.21. Seja $g \in L_{l o c}^{p}(\mathbb{R} ; V)$ uma função de translação limitada. Então:

1) Toda funçãoo $h \in \mathcal{H}(g)$ é de translação limitada e

$$
\|h\|_{L_{b}^{p}(\mathbb{R} ; V)} \leq\|g\|_{L_{b}^{p}(\mathbb{R} ; V)}
$$

para todo $h \in \mathcal{H}(g)$.

2) Se g é uma força externa regular no tempo. Então $h \in \mathcal{H}(g)$ também é regular no tempo.

3) Se g é uma força externa regular no espaço. Então $h \in \mathcal{H}(g)$ também é regular no espaço.

4) Se g é normal. Então toda função $h \in \mathcal{H}(g)$ é normal. Mais ainda,

$$
\sup _{h \in \mathcal{H}(g)}\left[\sup _{t \in \mathbb{R}} \int_{t}^{t+\tau}\|h(s)\|_{V}^{p} d s\right] \rightarrow 0
$$

quando $\tau \rightarrow 0$.

5) Seja g fortemente normal. Então toda função $h \in \mathcal{H}(g)$ é fortemente normal.

6) Seja $g$ fracamente normal. Então, para todo $h \in \mathcal{H}(g)$ e todo $\varepsilon>0$ existe $h_{\varepsilon} \in$ $\mathcal{H}\left(g_{\varepsilon}\right) \subset L_{b}^{p}\left(\mathbb{R} ; V_{\varepsilon}\right)$ tal que as desigualdades (2.2) e (2.3) são satisfeitas uniformemente com relação a $h \in \mathcal{H}(g)$. 
Os próximos resultados são fundamentais para demonstração da compacidade assintótica uniforme de alguns sistemas dinâmicos não autônomos. Nesta tese foram utilizado resultados sobre as força externas regulares no tempo como veremos no Capítulo 4 .

Proposição 2.4.22. Sejam $g$ e $g_{\varepsilon}$ forças externas pertencentes ao espaço de funções de translação limitada $L_{b}^{p}(\mathbb{R} ; V)$ tais que

$$
\left\|g-g_{\varepsilon}\right\|_{L_{b}^{p}(\mathbb{R} ; V)} \leq \varepsilon
$$

para algum $\varepsilon>0$. Então para todo $h \in \mathcal{H}(g)$ existe $h_{\varepsilon} \in \mathcal{H}\left(g_{\varepsilon}\right)$ tal que

$$
\left\|h-h_{\varepsilon}\right\|_{L_{b}^{p}(\mathbb{R} ; V)} \leq \varepsilon
$$

Corolário 2.4.23. Sejam $g \in L_{b}^{p}(\mathbb{R} ; V)$ regular no tempo e $h_{n} \in \mathcal{H}(g)$ tal que $h_{n} \rightarrow h$ em $L_{l o c, w}^{p}(\mathbb{R} ; V)$. Então, passando à uma subsequência se necessário, para todo $\varepsilon>0$ existe uma sequência $\varphi_{n} \in H_{b}^{k}(\mathbb{R} ; V)$, para qualquer $k \in \mathbb{N}$, tal que $\varphi_{n} \rightarrow \varphi$ em $H_{l o c, w}^{k}(\mathbb{R} ; V)$ e

$$
\left\|h_{n}-\varphi_{n}\right\|_{L_{b}^{p}(\mathbb{R} ; V)} \leq \varepsilon e\|h-\varphi\|_{L_{b}^{p}(\mathbb{R} ; V)} \leq \varepsilon
$$

Corolário 2.4.24. Sejam $g \in L_{b}^{p}(\mathbb{R} ; V)$ regular no espaço, $V=H^{s}(\Omega)$ com $s \in \mathbb{R} e$ $h_{n} \in \mathcal{H}(g)$ tal que $h_{n} \rightarrow h$ em $L_{l o c, w}^{p}(\mathbb{R} ; V)$. Então, passando à uma subsequência se necessário, para todo $\varepsilon>0$ existe uma sequência $\varphi_{n} \in L_{b}^{p}\left(\mathbb{R} ; H^{k}(\Omega)\right)$, para qualquer $k \in \mathbb{N}$, tal que $\varphi_{n} \rightarrow \varphi$ no espaço $L_{l o c, w}^{p}\left(\mathbb{R} ; H^{k}(\Omega)\right)$ e

$$
\left\|h_{n}-\varphi_{n}\right\|_{L_{b}^{p}(\mathbb{R} ; V)} \leq \varepsilon e\|h-\varphi\|_{L_{b}^{p}(\mathbb{R} ; V)} \leq \varepsilon
$$

\subsubsection{Processo de evolução e atratores uniformes}

Começamos relembrando o conceito de processo associado a um sistema não autônomo, agora definido sobre um espaço de Banach reflexivo.

Definição 2.4.25. Seja $W$ um espaço de Banach reflexivo. Uma família a dois parâmetros de operadores $U(t, \tau): W \rightarrow W, \tau \in \mathbb{R}$ e $t \geq \tau$, é um processo de evolução em $W$ se as seguintes propriedades são satisfeitas:

i) $U(\tau, \tau)=I d$, para todo $\tau \in \mathbb{R}$;

ii) $U(t, \tau)=U(t, s) U(s, \tau)$, sempre que $t \geq s \geq \tau$. 
Os processos de evolução são também frequentemente chamados de processos dinâmicos. Uma típica situação onde, naturalmente, aparece um processo é considerar o seguinte sistema não linear,

$$
\begin{aligned}
& \partial_{t} u=A(u)+g(t), \\
& \left.u\right|_{t=\tau}=u_{\tau},
\end{aligned}
$$

onde $A(u)$ é um operador não linear (não limitado), que não vamos especificar agora e $g(t)$ é uma força externa não autônoma tal que

$$
g \in L_{b}^{p}(\mathbb{R} ; V)
$$

para algum espaço de Banach reflexivo $V$ e para algum $1<p<\infty$.

Suponha que o sistema não linear (2.5) é bem posto globalmente em $W$, ou seja, para todo $\tau \in \mathbb{R}$ e todo $u_{\tau} \in W$ existe uma única solução $u(t) \in W, t \geq \tau$. Consequentemente o operador solução de (2.5) gera o seguinte processo em $W$,

$$
\begin{aligned}
& U(t, \tau): W \longrightarrow W \\
& u_{\tau} \longmapsto U(t, \tau) u_{\tau}=u(t),
\end{aligned}
$$

para todo $t \geq \tau$.

Para o estudo do comportamento das soluções a longo prazo, é conveniente considerar, não somente $g$ fixada, mas também estudar as soluções para todas as forças externas pertencentes à envoltória fraca $\mathcal{H}(g)$, isto é,

$$
\begin{aligned}
& \partial_{t} u=A(u)+h(t), h \in \mathcal{H}(g), \\
& \left.u\right|_{t=\tau}=u_{\tau} .
\end{aligned}
$$

Assim, associado ao sistema não autônomo (2.6), obtemos uma família de processos $U_{h}(t, \tau) u_{\tau}$, indexados em $h \in \mathcal{H}(g)$ e tal família satisfaz a chamada identidade de translação:

$$
U_{h}(t+s, \tau+s)=U_{T(s) h}(t, \tau)
$$

para todo $t \geq \tau$, todo $\tau \in \mathbb{R}$ e todo $s \in \mathbb{R}$. Esta identidade permite reduzir a dinâmica não autônoma em uma dinâmica autônoma, porém agindo num espaço maior, a saber, definimos tal espaço por

$$
\Phi=W \times \mathcal{H}(g)
$$


chamado de espaço de fase estendido associado ao sistema (2.6). Logo o semigrupo estendido definido sobre $\Phi$ é dado por

$$
\mathbb{S}(t)\left(u_{0}, h\right)=\left(U_{h}(t, 0) u_{0}, T(t) h\right),
$$

para $\left(u_{0}, h\right) \in \Phi$ e $t \geq 0$. Observe que, da identidade (2.7),

$$
\mathbb{S}(t): \Phi \longrightarrow \Phi
$$

e $\mathbb{S}\left(t_{1}+t_{2}\right)=\mathbb{S}\left(t_{1}\right) \mathbb{S}\left(t_{2}\right)$ para todos $t_{1}, t_{2} \geq 0$. Assim podemos falar sobre o atrator global do semigrupo $S(\cdot)$.

Definição 2.4.26. Um conjunto $\mathbb{A} \in \Phi$ é um atrator global fraco de $\mathbb{S}(t)$ se as seguintes propriedades são satisfeitas:

i) $\mathbb{A}$ é um conjunto compacto no espaço $\Phi$ munido com a topologia fraca dada pelo mergulho $\Phi \subset W_{w} \times L_{\text {loc, } w}^{p}(\mathbb{R} ; V)$. Vamos denotar $\Phi$ munido com esta topologia por $\Phi_{w}$.

ii) $\mathbb{A}$ é estritamente invariante, $\mathbb{S}(t) \mathbb{A}=\mathbb{A}$.

iii) $\mathbb{A}$ atrai as imagens de conjuntos limitados de $\Phi$ na topologia fraca, isto é, para todo limitado $B$ em $\Phi$ e toda vizinhança $\mathbb{O}(\mathbb{A})$ do conjunto $\mathbb{A}$ em $\Phi_{w}$, existe $T=T(B, \mathbb{O})$ tal que

$$
\mathbb{S}(t) B \subset \mathbb{O}(\mathbb{A}), \text { para todo } t \geq T \text {. }
$$

Então a projeção de $\mathbb{A}$ na componente $W$, é chamada atrator uniforme fraco associado a família $U_{h}(t, \tau): W \rightarrow W, t \geq \tau$ e $h \in \mathcal{H}(g)$, denotado por

$$
\mathcal{A}=\pi_{1} \mathbb{A}
$$

A fim de exibir um critério que verifica a existência de um atrator fraco, precisamos de algumas hipóteses naturais sobre o processo $U_{h}(t, \tau), h \in \mathcal{H}(g)$.

Definição 2.4.27. Uma família de processos $U_{h}(t, \tau): W \rightarrow W, h \in \mathcal{H}(g)$ é uniformemente dissipativa se a seguinte estimativa é satisfeita:

$$
\left\|U_{h}(t, \tau) u_{\tau}\right\|_{W} \leq Q\left(\left\|u_{\tau}\right\|_{W}\right) e^{-\beta(t-\tau)}+C_{*},
$$


para todo $\tau \in \mathbb{R}, t \geq \tau$ e todo $h \in \mathcal{H}(g)$, onde $\beta>0$ e $Q$ é uma função monótona que independe de $u_{\tau} \in W$, de $h \in \mathcal{H}(g)$ e $t \geq \tau$. Em particular, se a familia $U_{h}(t, \tau)$, $h \in \mathcal{H}(g)$ é uniformemente dissipativa, com relação à $h \in \mathcal{H}(g)$, podemos exibir um conjunto limitado $B_{0} \subset W$ tal que para qualquer $\tau \in \mathbb{R}$ e qualquer limitado $B \subset W$, existe $T_{0}=T_{0}(B, \tau) \geq \tau$ de forma que

$$
\bigcup_{h \in \mathcal{H}(g)} U_{h}(t, \tau) B \subset B_{0}, \text { para todo } t \geq T_{0} .
$$

Este conjunto limitado é chamado de conjunto uniformemente absorvente para família $U_{h}(t, \tau), h \in \mathcal{H}(g)$.

Definição 2.4.28. Dizemos que a família $U_{h}(t, \tau): W \rightarrow W$ é fracamente contínua se para quaisquer $t$ e $\tau$ fixos, as convergências fracas $u_{\tau}^{n} \rightarrow u$ em $W_{w}$ e $h_{n} \rightarrow h$ em $L_{l o c, w}^{p}(\mathbb{R} ; V)$ implicam que

$$
U_{h_{n}}(t, \tau) u_{\tau}^{n} \rightarrow U_{h}(t, \tau) u_{\tau}
$$

fracamente em $W$.

Com essas duas propriedades, podemos enunciar o seguinte critério.

Teorema 2.4.29. Seja $U_{h}(t, \tau): W \rightarrow W, h \in \mathcal{H}(g)$, uma família de processos associados ao sistema (2.6). Suponha que a família $U_{h}(t, \tau), h \in \mathcal{H}(g)$ é uniformemente dissipativa e fracamente contínua. Então o semigrupo estendido associado $\mathbb{S}: \Phi \rightarrow \Phi$ admite um atrator global fraco $\mathbb{A}$. Mais ainda o atrator uniforme fraco associado, $\pi_{1} \mathbb{A}=\mathcal{A}$ é gerado por todas as soluções limitadas de (2.6) com $h \in \mathcal{H}(g)$. Isto é,

$$
\mathcal{A}=\left.\bigcup_{h \in \mathcal{H}(g)} \mathcal{K}_{h}\right|_{t=0}
$$

Denotamos por $\mathcal{K}_{h} \subset L^{\infty}(\mathbb{R} ; V)$ o conjunto de todas as trajetórias $u(t)$ com $t \in \mathbb{R} d o$ sistema (2.6) que são globais e limitadas. Este conjunto é chamado de núcleo da equação (2.6) na terminologia introduzida por Vishik e Chepyzhov [14].

Observação 2.4.30. Existe uma definição intrínseca do atrator uniforme $\mathcal{A}$ que não se refere a redução do sistema não autônomo para um sistema autônomo. No entanto, do nosso ponto de vista a definição baseada na redução à um semigrupo é mais transparente, por isso vamos usá-la como definição principal nesta seção. A definição intrínseca pode ser encontrada no livro do Vishik e Chepyzhov, eles também mostram a equivalência das definições. 
Em geral mostrar a existência de um atrator uniforme fraco não apresenta grandes dificuldades, por isso vamos em busca de atratores uniformes em topologias mais restritivas, ou seja, atração na topologia forte. Com isso surge a seguinte definição.

Definição 2.4.31. Um conjunto $\mathbb{A} \subset \Phi$ é um atrator global forte de $\mathbb{S}(t)$, se satisfaz as seguinte propriedades:

i) $\mathbb{A}$ é compacto em $\Phi$ munido com a topologia forte induzida pelo mergulho $\Phi \subset W \times$ $L_{l o c, w}^{p}(\mathbb{R} ; V)$. Denotamos por $\Phi_{s}$ o espaço $\Phi$ munido com esta topologia.

ii) $\mathbb{A}$ é estritamente invariante, $\mathbb{S}(t) \mathbb{A}=\mathbb{A}$.

iii) $\mathbb{A}$ atrai imagens de limitados de $\Phi$ na topologia forte, ou seja, para todo limitado $B$ em $\Phi$ e toda vizinhança $\mathbb{O}(\mathbb{A})$ em $\Phi_{s}$, existe $T=T(B, \mathbb{O})$ tal que

$$
\mathbb{S}(t) B \subset \mathbb{O}(\mathbb{A}), \text { para todo } t \geq T \text {. }
$$

Então, a projeção de $\mathbb{A}$ na primeira componente $W$ é chamada de atrator uniforme forte associado a família $U_{h}(t, \tau), h \in \mathcal{H}(g)$. Denotado por

$$
\mathcal{A}=\pi_{1} \mathbb{A}
$$

Antes de exibirmos um critério para existência de um atrator uniforme forte, precisamos de uma propriedade adicional na família de processos $U_{h}(t, \tau)$.

Definição 2.4.32. Uma família de processos $U_{h}(t, \tau): W \rightarrow W, h \in \mathcal{H}(g)$ é uniformemente assintóticamente compacta, se para toda sequência $\left\{u_{n}\right\} \subset W$ limitada, toda sequência de forças externas $\left\{h_{n}\right\} \subset \mathcal{H}(g)$ e toda sequência $t_{n} \geq \tau_{n}$ com $t_{n}-\tau_{n} \rightarrow \infty$, então a sequência

$$
\left\{U_{h_{n}}\left(t_{n}, \tau_{n}\right) u_{n}\right\} \subset W
$$

é pré-compacta em $W$.

Com esta definição, podemos exibir um critério para existência de um atrator uniforme forte.

Teorema 2.4.33. Suponha que as hipótese do Teorema 2.4.29 são satisfeitas e que a família de processos $U_{h}(t, \tau), h \in \mathcal{H}(g)$, seja uniformemente assintóticamente compacta. Então o atrator uniforme fraco, $\pi_{1} \mathbb{A}=\mathcal{A}$ construído no Teorema 2.4.29 é um atrator uniforme forte em $W$, no sentido da Definição 2.4.31. 
Observação 2.4.34. Como podemos ver, para verificar a existência de um atrator forte, basicamente, precisamos verificar a compacidade assintótica, por isso vamos nos concentrar a seguir na verificação desta propriedade. Note, também, que a condição de compacidade assintótica pode ser ligeiramente simplificada utilizando a identidade de translação (2.7). Ou seja, é suficiente verificar que para qualquer sequência limitada $\left\{u_{n}\right\} \subset W$, qualquer sequência de forças externas $\left\{h_{n}\right\} \in \mathcal{H}(g)$ e qualquer sequência $t_{n} \rightarrow \infty$, a sequência

$$
\left\{U_{h_{n}}\left(t_{n}, 0\right) u_{n}\right\} \subset W
$$

é pré-compacta em $W$.

Na sequência, vamos exibir um critério para compacidade assintótica uniforme.

\subsubsection{Critério para compacidade assintótica}

Em geral, verificar a compacidade assintótica uniforme de uma família de processos pela definição não é uma tarefa muito fácil. Nesta seção daremos a definição de função contrativa uniforme e finalizaremos com uma condição suficiente para compacidade assintótica uniforme, que pode ser encontrada em alguns trabalhos. Ver [33, 42, 44].

Definição 2.4.35. Seja $W$ um espaço de Banach, $B$ um conjunto limitado de $W$ e $\mathcal{H}(g)$ o espaço de símbolos. Uma função $\Phi(\cdot, \cdot ; \cdot, \cdot)$ definida sobre $(W \times W) \times(\mathcal{H}(g) \times \mathcal{H}(g))$ é contrativa sobre $B \times B$ se para toda sequência $\left\{u_{n}\right\} \subset B$ e toda sequência $\left\{h_{n}\right\} \subset \mathcal{H}(g)$ existe uma subsequência $\left\{u_{n_{k}}\right\} \subset\left\{u_{n}\right\}$ e uma subsequência $\left\{h_{n_{k}}\right\} \subset\left\{h_{n}\right\}$ tais que

$$
\lim _{k \rightarrow \infty} \lim _{l \rightarrow \infty} \Phi\left(x_{n_{k}}, x_{n_{l}} ; h_{n_{k}}, h_{n_{l}}\right)=0
$$

Teorema 2.4.36. Seja $U_{h}(t, \tau), h \in \mathcal{H}(g)$ uma família de processos sobre um espaço de Banach $W$ que admite um conjunto uniformemente absorvente $B_{0} \subset W$ e limitado em $W$ (uniformemente dissipativo). Mais ainda, suponha que para todo $\varepsilon>0$ existe $T=T\left(B_{0}, \varepsilon\right)$ e uma função contrativa $\Phi_{T}$ em $B_{0} \times B_{0}$, tal que

$$
\left\|U_{\sigma_{1}}(T, 0) u-U_{\sigma_{2}}(T, 0) v\right\| \leq \varepsilon+\Phi\left(u, v ; \sigma_{1}, \sigma_{2}\right)
$$

para todos $u, v \in B_{0}$ e todos $\sigma_{1}, \sigma_{2} \in \mathcal{H}(g)$. Então $U_{h}(t, \tau), h \in \mathcal{H}(g)$ é uniformemente assintoticamente compacto em $W$. 
Demonstração. Pela Observação 2.4.34, precisamos mostrar que dada qualquer sequência limitada $\left\{u_{n}\right\} \subset W$, qualquer sequência de forças $\left\{h_{n}\right\} \subset \mathcal{H}(g)$ e $t_{n} \rightarrow \infty$ então

$$
\left\{U_{h_{n}}\left(t_{n}, 0\right) u_{n}\right\} \subset W
$$

é pré-compacta em $W$. De fato, considere $\varepsilon_{m} \rightarrow 0$ quando $m \rightarrow \infty$ e tal que $\varepsilon_{m}>0$.

Para $\varepsilon_{1}>0$, pela hipótese, existe $T_{1}\left(B_{0}, \varepsilon_{1}\right)>0$ e uma função contrativa $\Phi_{T_{1}}$ em $B_{0} \times B_{0}$ tal que

$$
\left\|U_{\sigma_{1}}\left(T_{1}, 0\right) u-U_{\sigma_{2}}\left(T_{1}, 0\right) v\right\|_{W} \leq \frac{\varepsilon_{1}}{2}+\Phi_{T_{1}}\left(u, v ; \sigma_{1}, \sigma_{2}\right)
$$

para todos $u, v \in B_{0}$ e todos $\sigma_{1}, \sigma_{2} \in \mathcal{H}(g)$.

Como $B=\left\{u_{n}, n \in \mathbb{N}\right\}$ é limitado em $W$ e $t_{n} \rightarrow \infty$, existe $n_{0} \in \mathbb{N}$ tal que

$$
t_{n} \geq t_{n}-T_{1} \geq 0 \quad \text { e } \quad U_{h_{n}}\left(t_{n}-T_{1}, 0\right) u_{n} \in B_{0}
$$

para todo $n \geq n_{0}$. Passando à uma subsequência se necessário, podemos assumir que

$$
y_{n}^{T_{1}}=U_{h_{n}}\left(t_{n}-T_{1}, 0\right) u_{n} \in B_{0}, \quad \text { para todo } n \in \mathbb{N}
$$

Defina $\tilde{h}_{n}^{T_{1}}=T\left(T_{1}-t_{n}\right) h_{n} \in \mathcal{H}(g)$ e note que a sequência $\left\{y_{n}^{T_{1}}\right\}$ depende das sequências $\left\{h_{n}\right\},\left\{t_{n}\right\}$ e $\left\{u_{n}\right\}$, e a sequência $\left\{\tilde{h}_{n}^{T_{1}}\right\}$ depende de $\left\{h_{n}\right\}$ e $\left\{t_{n}\right\}$. Portanto, como $\Phi_{T_{1}}$ é contrativa, existem subsequências $\left\{y_{n}^{T_{1}, 1}\right\}$ de $\left\{y_{n}^{T_{1}}\right\}$ e $\left\{\tilde{h}_{n}^{T_{1}, 1}\right\}$ de $\left\{\tilde{h}_{n}^{T_{1}}\right\}$ tais que

$$
\Phi_{T_{1}}\left(y_{n}^{T_{1}, 1}, y_{m}^{T_{1}, 1} ; \tilde{h}_{n}^{T_{1}, 1}, \tilde{h}_{m}^{T_{1}, 1}\right) \leq \frac{\varepsilon_{1}}{2}
$$

para todos $m, n \in \mathbb{N}$.

Observação 2.4.37. A priori, as subsequências $\left\{h_{n}^{1}\right\}$ de $\left\{h_{n}\right\},\left\{t_{n}^{1}\right\}$ de $\left\{t_{n}\right\}$ e $\left\{u_{n}^{1}\right\}$ de $\left\{u_{n}\right\}$ que definem $\left\{y_{n}^{T_{1}, 1}\right\}$, não precisam, necessariamente serem as mesmas subsequências que definem $h_{n}^{T_{1}, 1}$, porém para que possamos continuar a demonstração, precisamos supor que as subsequências $\left\{y_{n}^{T_{1}, 1}\right\}$ e $\left\{\tilde{h}_{n}^{T_{1}, 1}\right\}$ são definidas pelas mesmas subsequências $\left\{h_{n}^{1}\right\}$, $\left\{t_{n}^{1}\right\}$ e $\left\{u_{n}^{1}\right\}$. Veremos que na prática, as funções contrativas obtidas irão satisfazer essa propriedade. 
Assim, sendo $y_{n}^{T_{1}, 1}=U_{h_{n}^{1}}\left(t_{n}^{1}-T_{1}, 0\right) u_{n}^{1}$ e $\tilde{h}_{n}^{T_{1}, 1}=T\left(T_{1}-t_{n}^{1}\right) h_{n}^{1}$, então

$$
\begin{aligned}
& \left\|U_{h_{n}^{1}}\left(t_{n}^{1}, 0\right) u_{n}^{1}-U_{h_{m}^{1}}\left(t_{m}^{1}, 0\right) u_{m}^{1}\right\|_{W} \\
& \quad=\left\|U_{h_{n}^{1}}\left(t_{n}^{1}, t_{n}^{1}-T_{1}\right) U_{h_{n}^{1}}\left(t_{n}^{1}-T_{1}, 0\right) u_{n}^{1}-U_{h_{m}^{1}}\left(t_{m}^{1}, t_{m}^{1}-T_{1}\right) U_{h_{m}^{1}}\left(t_{m}^{1}-T_{1}, 0\right) u_{m}^{1}\right\|_{W} \\
& \quad=\left\|U_{h_{n}^{1}}\left(t_{n}^{1}, t_{n}^{1}-T_{1}\right) y_{n}^{T_{1}, 1}-U_{h_{m}^{1}}\left(t_{m}^{1}, t_{m}^{1}-T_{1}\right) y_{m}^{T_{1}, 1}\right\|_{W} \\
& \quad=\left\|U_{T\left(T_{1}-t_{n}^{1}\right) h_{n}^{1}}\left(T_{1}, 0\right) y_{n}^{T_{1}, 1}-U_{T\left(T_{1}-t_{m}^{1}\right) h_{m}^{1}}\left(T_{1}, 0\right) y_{m}^{T_{1}, 1}\right\|_{W} \\
& \quad=\left\|U_{\tilde{h}_{n}^{T_{1}}, 1}\left(T_{1}, 0\right) y_{n}^{T_{1}, 1}-U_{\tilde{h}_{m}^{T_{1}, 1}}\left(T_{1}, 0\right) y_{m}^{T_{1}, 1}\right\|_{W} \leq \frac{\varepsilon_{1}}{2}+\Phi_{T_{1}}\left(y_{n}^{T_{1}, 1}, y_{m}^{T_{1}, 1} ; \tilde{h}_{n}^{T_{1}, 1}, \tilde{h}_{m}^{T_{1}, 1}\right) .
\end{aligned}
$$

Consequentemente, de (2.8),

$$
\left\|U_{h_{n}^{1}}\left(t_{n}^{1}, 0\right) u_{n}^{1}-U_{h_{m}^{1}}\left(t_{m}^{1}, 0\right) u_{m}^{1}\right\|_{W} \leq \varepsilon_{1}, \quad \text { para todos } n, m \in \mathbb{N} .
$$

Procedendo analogamente, para $\varepsilon_{2}>0$ existe $T_{2}\left(B_{0}, \varepsilon_{2}\right)$ e uma função contrativa $\Phi_{T_{2}}$ tal que

$$
\left\|U_{\sigma_{1}}\left(T_{2}, 0\right) u-U_{\sigma_{2}}\left(T_{2}, 0\right) v\right\|_{W} \leq \frac{\varepsilon_{2}}{2}+\Phi_{T_{2}}\left(u, v ; \sigma_{1}, \sigma_{2}\right),
$$

para todos $u, v \in B_{0}$ e todos $\sigma_{1}, \sigma_{2} \in \mathcal{H}(g)$.

Como $t_{n}^{1} \rightarrow \infty$ e a subsequência $\left\{u_{n}^{1}\right\}$ é um subconjunto de $B$, existe $n_{0} \in \mathbb{N}$ tal que

$$
t_{n}^{1} \geq t_{n}^{1}-T_{2} \geq 0 \quad \text { e } \quad U_{h_{n}^{1}}\left(t_{n}^{1}-T_{2}, 0\right) u_{n}^{1} \in B_{0},
$$

para todo $n \geq n_{0}$. Passando para uma subsequência se necessário, podemos supor que

$$
y_{n}^{T_{2}}=U_{h_{n}^{1}}\left(t_{n}^{1}-T_{2}, 0\right) u_{n}^{1} \in B_{0} \quad \text { para todo } n \in \mathbb{N} .
$$

Defina $\tilde{h}_{n}^{T_{2}}=T\left(T_{2}-t_{n}^{1}\right) h_{n}^{1} \in \mathcal{H}(g)$. Assim pelo fato de que $\Phi_{T_{2}}$ é contrativa, existem subsequências $\left\{h_{n}^{2}\right\}$ de $\left\{h_{n}^{1}\right\},\left\{t_{n}^{2}\right\}$ de $\left\{t_{n}^{1}\right\}$ e $\left\{u_{n}^{2}\right\}$ de $\left\{u_{n}^{1}\right\}$ tais que

$$
\Phi_{T_{2}}\left(y_{n}^{T_{2}, 2}, y_{m}^{T_{2}, 2} ; \tilde{h}_{n}^{T_{2}, 2}, \tilde{h}_{m}^{T_{2}, 2}\right) \leq \frac{\varepsilon_{2}}{2} \quad \text { para todos } \quad n, m \in \mathbb{N},
$$

onde $y_{n}^{T_{2}, 2}=U_{h_{n}^{2}}\left(t_{n}^{2}-T_{2}, 0\right) u_{n}^{2}$ é uma subsequência de $y_{n}^{T_{2}}$ e $\tilde{h}_{n}^{T_{2}, 2}=T\left(T_{2}-t_{n}^{2}\right) h_{n}^{2}$ é uma subsequência de $h_{n}^{T_{2}}$. Portanto

$$
\begin{aligned}
\left\|U_{h_{n}^{2}}\left(t_{n}^{2}, 0\right) u_{n}^{2}-U_{h_{m}^{2}}\left(t_{m}^{2}, 0\right) u_{m}^{2}\right\|_{W} & =\left\|U_{\tilde{h}_{n}^{T_{2}, 2}}\left(T_{2}, 0\right) y_{n}^{T_{2}, 2}-U_{\tilde{h}_{m}^{T_{2}, 2}}\left(T_{2}, 0\right) y_{m}^{T_{2}, 2}\right\|_{W} \\
& \leq \frac{\varepsilon_{2}}{2}+\Phi_{T_{2}}\left(y_{n}^{T_{2}, 2}, y_{m}^{T_{2}, 2} ; \tilde{h}_{n}^{T_{2}, 2}, \tilde{h}_{m}^{T_{2}, 2}\right) \\
& \leq \varepsilon_{2},
\end{aligned}
$$

para todos $n, m \in \mathbb{N}$. 
Repetindo este argumento $k$ vezes, vamos obter subsequências $\left\{h_{n}^{k}\right\}$ de $\left\{h_{n}^{k-1}\right\},\left\{t_{n}^{k}\right\}$ de $\left\{t_{n}^{k-1}\right\}$ e $\left\{u_{n}^{k}\right\}$ de $\left\{u_{n}^{k-1}\right\}$ tais que

$$
\left\|U_{h_{n}^{k}}\left(t_{n}^{k}, 0\right) u_{n}^{k}-U_{h_{m}^{k}}\left(t_{m}^{k}, 0\right) u_{m}^{k}\right\|_{W} \leq \varepsilon_{k}
$$

para todos $n, m \in \mathbb{N}$.

Finalmente, defina subsequência diagonal $\left\{U_{h_{n}^{n}}\left(t_{n}^{n}, 0\right) u_{n}^{n}\right\}$ de $\left\{U_{h_{n}}\left(t_{n}, 0\right) u_{n}\right\}$ e observe que

$$
\left\|U_{h_{n}^{n}}\left(t_{n}^{n}, 0\right) u_{n}^{n}-U_{h_{m}^{m}}\left(t_{m}^{m}, 0\right) u_{m}^{m}\right\|_{W} \leq \varepsilon_{m}
$$

desde que $n \geq m$. Como $\varepsilon_{m} \rightarrow 0$, concluímos que a subsequência diagonal $\left\{U_{h_{n}^{n}}\left(t_{n}^{n}, 0\right) u_{n}^{n}\right\}$ é uma sequência de Cauchy em $W$ e portanto convergente em $W$. Isto prova que as sequência $\left\{U_{h_{n}}\left(t_{n}, 0\right) u_{n}\right\}$ é pré-compacta em $W$, como queríamos. 


\section{Atrator pullback para equação da}

\section{onda com fronteira acústica}

\subsection{Introdução}

Neste capítulo estudamos uma classe da equação da onda com condição de fronteira da acústica. Primeiramente, vamos modelar fisicamente este tipo de problema. Este modelo descreve o movimento de um onda acústica em um fluido ocupando uma região limitada $\Omega$ do $\mathbb{R}^{3}$. Se $u$ é a velocidade potencial, então $-\nabla u$ é o campo velocidade da partícula e assim $u$ deve satisfazer a seguinte equação da onda

$$
u_{t t}-\Delta u=0 \quad \text { em } \quad \Omega
$$

Agora, suponha que a fronteira de $\Omega$, denotada por $\Gamma$, não é rígida e esta sujeita a pequenas oscilações. Vamos assumir que cada ponto de $\Gamma$ reage à pressão causada pela onda acústica como um oscilador harmônico amortecido e mais, $\Gamma$ é localmente reagente, o que significa que cada um de seus pontos age, devido a pressão da onda acústica, de modo independente um do outro. Assim, denotando por $\delta$ o deslocamento da fronteira na direção normal, obtemos que $\delta$ deve satisfazer a seguinte equação

$$
\delta_{t t}+\delta_{t}+\delta=-u_{t} \quad \text { em } \quad \Gamma
$$

Vamos assumir também uma condição de impenetrabilidade da fronteira, isto é, admite-se que há compatibilidade entre a velocidade normal da fronteira, e a velocidade normal do fluido. Logo devemos ter que

$$
\delta_{t}=\partial_{\mathbf{n}} u \quad \text { em } \quad \Gamma,
$$

onde $\mathbf{n}$ é o vetor normal unitário em $\Gamma$, exterior à $\Omega$. 
As equações (3.1) e (3.2) são chamadas de condições de fronteira da acústica e que foram inicialmente propostas por Beale e Rosencrans [4]. Este modelo foi estudado por muitos autores e extendido de várias maneiras. Por exemplo $[3,5,6,15,21,22,23,24$, $25,27,28,30,38,43]$.

Estamos interessados no comportamento a longo prazo do seguinte problema. Seja $\Omega \subset \mathbb{R}^{3}$ um domínio limitado com fronteira regular $\Gamma$ e $\tau \in \mathbb{R}$ um tempo inicial e considere equação da onda não autônoma com condição de fronteira da acústica

$$
\begin{aligned}
& u_{t t}-\Delta u+\omega u_{t}+u+f(u)=h(x, t), \quad x \in \Omega, \quad t \geq \tau, \\
& \delta_{t t}+\nu \delta_{t}+\delta=-u_{t}, \quad x \in \Gamma, \quad t \geq \tau, \\
& \delta_{t}=\partial_{\mathbf{n}} u, \quad x \in \Gamma, \quad t \geq \tau,
\end{aligned}
$$

onde $\omega, \nu>0$ são coeficientes de damping, $f$ é um termo não linear e $h$ é uma força externa dependendo do tempo $t$. As condições iniciais são denotadas por

$$
\begin{aligned}
& u(x, \tau)=u_{\tau}^{0}(x), \quad u_{t}(x, \tau)=u_{\tau}^{1}(x), \quad x \in \Omega, \\
& \delta(x, \tau)=\delta_{\tau}^{0}(x), \quad \delta_{t}(x, \tau)=\delta_{\tau}^{1}(x), \quad x \in \Gamma .
\end{aligned}
$$

Este capítulo esta organizado da seguinte maneira. Na Seção 3.2 fixaremos as hipóteses sobre o termo não linear e sobre a força externa da equação (3.3). Na Seção 3.3 exibiremos o resultado sobre a existência de soluções do problema (3.3)-(3.7), via método de semigrupos. Na Seção 3.4 apresentaremos estimativas da energia, associada ao problema, que serão cruciais na prova da existência do atrator pullback. Na Seção 3.5 mostraremos a existência de um absorvente e a compacidade assintótica no sentido pullback do processo de evolução associado ao problema (3.3)-(3.7) e, consequentemente, a existência do atrator pullback. Por fim, iremos considerar uma variação da força externa $h$ substituindo-a por $\varepsilon h$ e mostraremos uma certa continuidade da família de atratores quanto $\varepsilon \rightarrow 0$.

\subsection{Hipótese e notações iniciais}

Iniciaremos com as hipóteses sobre as funções $f$ e $h$. Vamos considerar o termo não linear $f \in C^{1}(\mathbb{R})$ tal que

$$
\left|f^{\prime}(u)\right| \leq C\left(1+|u|^{2}\right), \quad \forall u \in \mathbb{R}
$$


para alguma constante $C>0$, e existe $\beta \in(0,1)$ e $m_{f}>0$ tal que

$$
F(u) \geq-\frac{\beta}{2} u^{2}-m_{f} \quad \text { e } \quad f(u) u-F(u) \geq-\frac{\beta}{2} u^{2}-m_{f}, \quad \forall u \in \mathbb{R},
$$

onde $F(u)=\int_{0}^{u} f(s) d s$. Com respeito a força externa não autônoma assumimos que existe um $\sigma_{0}>0$ (à ser fixado no Teorema 3.5.1) de modo que

$$
h \in L_{l o c}^{2}\left(\mathbb{R} ; L^{2}(\Omega)\right) \quad \text { e } \quad \int_{-\infty}^{t} e^{-\sigma_{0}(t-s)}\|h(s)\|_{L^{2}(\Omega)}^{2} d s<\infty, \quad \forall t \in \mathbb{R} .
$$

Observação 3.2.1. (a) A condição (3.8) significa que $f$ permite um crescimento crítico do tipo $|u|^{3}$. A condição (3.9) é usual e é satisfeita se

$$
\liminf _{|u| \rightarrow \infty} f^{\prime}(u)>-1 .
$$

(b) A segunda condição em (3.10) parece não ser usual, pois depende de $t$, mas esta condição é satisfeita se

$$
\int_{-\infty}^{0} e^{\sigma_{0} s}\|h(s)\|_{L^{2}(\Omega)}^{2} d s<\infty
$$

como considerado em [9, 26].

\subsection{Existência e unicidade}

A boa colocação do problema (3.3)-(3.7) foi estudada em [21] no caso autônomo, com $h=0$, usando a teoria de semigrupo. Um resultado análogo de existência e unicidade desse problema, agora com uma força externa dependendo do tempo $h=h(x, t)$, segue usando argumentos similares.

Iniciaremos definindo os seguintes espaço de Hilbert

$$
\mathcal{H}=H^{1}(\Omega) \times L^{2}(\Omega) \times L^{2}(\Gamma) \times L^{2}(\Gamma)
$$

e (escrevendo $\left.w=\left(w_{1}, w_{2}, w_{3}, w_{4}\right)\right)$

$$
\mathcal{H}_{1}=\left\{w \in H^{2}(\Omega) \times H^{1}(\Omega) \times H^{1 / 2}(\Gamma) \times H^{1 / 2}(\Gamma) \mid \partial_{\mathbf{n}} w_{1}=w_{4}\right\},
$$

equipados com as seguintes normas usuais

$$
\left\|\left(w_{1}, w_{2}, w_{3}, w_{4}\right)\right\|_{\mathcal{H}}^{2}=\left\|w_{1}\right\|_{H^{1}(\Omega)}^{2}+\left\|w_{2}\right\|_{L^{2}(\Omega)}^{2}+\left\|w_{3}\right\|_{L^{2}(\Gamma)}^{2}+\left\|w_{4}\right\|_{L^{2}(\Gamma)}^{2}
$$

e

$$
\left\|\left(w_{1}, w_{2}, w_{3}, w_{4}\right)\right\|_{\mathcal{H}_{1}}^{2}=\left\|w_{1}\right\|_{H^{2}(\Omega)}^{2}+\left\|w_{2}\right\|_{H^{1}(\Omega)}^{2}+\left\|w_{3}\right\|_{H^{1 / 2}(\Gamma)}^{2}+\left\|w_{4}\right\|_{H^{1 / 2}(\Gamma)}^{2}
$$

respectivamente. 
Definição 3.3.1. Dizemos que um par de funções $u: \Omega \times[\tau, \infty) \rightarrow \mathbb{R}, \delta: \Gamma \times[\tau, \infty) \rightarrow \mathbb{R}$ é uma solução fraca do sistema (3.3)-(3.7), desde que, dado $T>\tau$ arbitrário,

- $u \in C\left([\tau, T] ; H^{1}(\Omega)\right), u_{t} \in C\left([\tau, T] ; L^{2}(\Omega)\right), u_{t t} \in L^{\infty}\left([\tau, T] ; H^{-1}(\Omega)\right)$;

- $\delta, \delta_{t} \in C\left([\tau, T] ; L^{2}(\Gamma)\right), u_{t} \in L^{2}\left([\tau, T] ; L^{2}(\Gamma)\right)$;

- Para todo $\phi \in H^{1}(\Omega)$, todo $\psi \in L^{2}(\Gamma)$, nós temos:

$$
\begin{aligned}
& \left(u_{t t}, \phi\right)+(\nabla u, \nabla \phi)_{L^{2}(\Omega)}+\left(\omega u_{t}+u+f(u), \phi\right)_{L^{2}(\Omega)}=(h, \phi)_{L^{2}(\Omega)}+\left(\delta_{t}, \phi\right)_{L^{2}(\Gamma)} \\
& \left(\delta_{t t}, \psi\right)_{L^{2}(\Gamma)}+\left(\nu \delta_{t}, \psi\right)_{L^{2}(\Gamma)}+(\delta, \psi)_{L^{2}(\Gamma)}=\left(u_{t}, \psi\right)_{L^{2}(\Gamma)} \\
& u(\tau)=u_{\tau}^{0}, u_{t}(\tau)=u_{\tau}^{1}, \delta(\tau)=\delta_{\tau}^{0}, \delta_{t}(\tau)=\delta_{\tau}^{1}
\end{aligned}
$$

Observação 3.3.2. O próximo resultado irá mostrar que o problema de Cauchy equivalente ao sistema (3.3)-(3.7) tem uma única solução contínua no tempo no sentido generalizado, denotada por $z$, isto é, podemos escrever $z(t)=\lim _{n \rightarrow \infty} z_{n}(t)$ onde $z_{n}$ é a solução do problema de Cauchy com $z_{n}(0)$ pertencente ao domínio do operador $A$ (à definir) $e$ está convergência é na topologia forte de $\mathcal{H}$. Tais soluções $z_{n}$ são fortes ou regulares (satisfazem (3.3)-(3.5) quase sempre, com veremos a seguir).

Teorema 3.3.3. Sob as hipóteses (3.8)-(3.10). Dados um tempo inicial $\tau \in \mathbb{R}$ e um dado inicial $z_{\tau}=\left(u_{\tau}^{0}, u_{\tau}^{1}, \delta_{\tau}^{0}, \delta_{\tau}^{1}\right) \in \mathcal{H}$, o problema (3.3)-(3.7) tem uma única solução generalizada $z=\left(u, u_{t}, \delta, \delta_{t}\right)$ satisfazendo

$$
z \in C^{0}([\tau, \infty) ; \mathcal{H})
$$

Se o dado inicial $z_{\tau}=\left(u_{\tau}^{0}, u_{\tau}^{1}, \delta_{\tau}^{0}, \delta_{\tau}^{1}\right) \in \mathcal{H}_{1}$ e $h \in H_{l o c}^{1}\left(\mathbb{R} ; L^{2}(\Omega)\right)$ então a solução acima é uma solução regular, ou seja,

$$
z \in C^{0}\left([\tau, \infty) ; \mathcal{H}_{1}\right) \cap C^{1}([\tau, \infty) ; \mathcal{H})
$$

Além disso, se $z^{i}(t)=\left(u^{i}(t), u_{t}^{i}(t), \delta^{i}(t), \delta_{t}^{i}(t)\right), i=1,2$, são duas soluções generalizadas do problema (3.3)-(3.7), então para todo $T>\tau$,

$$
\left\|z^{1}(t)-z^{2}(t)\right\|_{\mathcal{H}}^{2} \leq e^{c_{0}(T-\tau)}\left\|z^{1}(\tau)-z^{2}(\tau)\right\|_{\mathcal{H}}^{2},
$$

para todo $t \in[\tau, T]$, onde $c_{0}$ é uma constante que depende dos dados iniciais. 
Demonstração. A prova desse teorema consiste em escrever nosso problema (3.3)-(3.7) em um problema de Cauchy equivalente, aplicar o Teorema 1.0.15 para obter uma solução local e posteriormente, com um argumento de contradição, obter uma solução global. Por fim mostrar a desigualdade (3.11) que implica dependência contínua dos dados iniciais.

Dividimos essa demonstração em algumas etapas.

Etapa 1: O problema (3.3)-(3.7) é equivalente a um problema de Cauchy.

Observe que podemos reescrever o problema (3.3)-(3.7) equivalentemente como o seguinte problema de Cauchy em $\mathcal{H}$,

$$
\left\{\begin{array}{l}
\frac{d z}{d t}=A z+\mathcal{F} z+\mathcal{G}, \\
z(\tau)=z_{\tau},
\end{array}\right.
$$

onde, denotando $z=\left(z_{1}, z_{2}, z_{3}, z_{4}\right)^{\top}$,

$$
A z=\left[\begin{array}{c}
z_{2} \\
\Delta z_{1}-z_{1}-\omega z_{2} \\
z_{4} \\
-z_{2}-z_{3}-\nu z_{4}
\end{array}\right]
$$

com

$$
\begin{gathered}
D(A)=\left\{\left(z_{1}, z_{2}, z_{3}, z_{4}\right) \in \mathcal{H} ; \Delta z_{1} \in L^{2}(\Omega), z_{2} \in H^{1}(\Omega), \partial_{\mathbf{n}} z_{1}=z_{4}\right\}, \\
\mathcal{F} z=\left(0,-f\left(z_{1}\right), 0,0\right) \text { e } \mathcal{G}=(0, h, 0,0) .
\end{gathered}
$$

Como observado em $[6,21]$, na definição de $D(A)$, a condição $\partial_{\mathbf{n}} z_{1}=z_{4}$ é entendida no sentido fraco, isto é,

$$
\int_{\Omega}\left(\Delta z_{1} \varphi+\nabla z_{1} \nabla \varphi\right) d x=\int_{\Gamma} z_{4} \varphi d \gamma, \quad \forall \varphi \in H^{1}(\Omega)
$$

Etapa 2: O operador $A$ é monótono.

De fato, dado $z=\left(z_{1}, z_{2}, z_{3}, z_{4}\right) \in D(A)$ temos que

$$
\begin{aligned}
(A z, z)_{\mathcal{H}}= & \left(z_{2}, z_{1}\right)_{H^{1}(\Omega)}+\left(\Delta z_{1}-z_{1}-\omega z_{2}, z_{2}\right)_{L^{2}(\Omega)} \\
& +\left(z_{4}, z_{3}\right)_{L^{2}(\Gamma)}+\left(-z_{2}-z_{3}-\nu z_{4}, z_{4}\right)_{L^{2}(\Gamma)} \\
= & -\omega\left\|z_{2}\right\|_{L^{2}(\Omega)}^{2}-\nu\left\|z_{4}\right\|_{L^{2}(\Gamma)}^{2}+\left(z_{2}, z_{1}\right)_{H^{1}(\Omega)} \\
& +\left(\Delta z_{1}, z_{2}\right)_{L^{2}(\Omega)}-\left(z_{1}, z_{2}\right)_{L^{2}(\Omega)}-\left(z_{2}, z_{4}\right)_{L^{2}(\Gamma)} .
\end{aligned}
$$


Usando o sentido fraco da derivada normal (3.13), obtemos que

$$
(A z, z)_{\mathcal{H}}=-\omega\left\|z_{2}\right\|_{L^{2}(\Omega)}^{2}-\nu\left\|z_{4}\right\|_{L^{2}(\Gamma)}^{2} \leq 0 .
$$

Portanto o operador $A$ é monótono.

Etapa 3: O operador $A$ é maximal, ou seja, $R(I-A)=\mathcal{H}$.

De fato, dado $\left(f_{1}, f_{2}, f_{3}, f_{4}\right) \in \mathcal{H}$ devemos mostrar que existe $\left(z_{1}, z_{2}, z_{3}, z_{4}\right) \in D(A)$ tal que

$$
(I-A)\left(z_{1}, z_{2}, z_{3}, z_{4}\right)^{\top}=\left(f_{1}, f_{2}, f_{3}, f_{4}\right)^{\top}
$$

Isto é,

$$
\left[\begin{array}{c}
z_{1}-z_{2} \\
z_{2}-\Delta z_{1}+z_{1}+\omega z_{2} \\
z_{3}-z_{4} \\
z_{4}+z_{2}+z_{3}+\nu z_{4}
\end{array}\right]=\left[\begin{array}{c}
f_{1} \\
f_{2} \\
f_{3} \\
f_{4}
\end{array}\right]
$$

Para isso consideramos em $H^{1}(\Omega)$ a estrutura dada pelo seguinte produto interno:

$$
(((u, v)))=(\nabla u, \nabla v)_{L^{2}(\Omega)}+(2+\omega)(u, v)_{L^{2}(\Omega)}+\frac{1}{2+\nu}(u, v)_{L^{2}(\Gamma)},
$$

para todos $u, v \in H^{1}(\Omega)$. Observe que, pela continuidade da aplicação traço, a norma induzida por esse produto interno é equivalente a norma usual em $H^{1}(\Omega)$. Consequentemente $H^{1}(\Omega)$ munido com o produto interno $(((\cdot, \cdot)))$ é um espaço de Hilbert. Definindo

$$
\begin{aligned}
\chi: H^{1}(\Omega) & \longrightarrow \mathbb{R} \\
v & \longmapsto\langle\chi, v\rangle=\left(\xi_{1}, v\right)_{L^{2}(\Omega)}+\left(\xi_{2}, v\right)_{L^{2}(\Gamma)},
\end{aligned}
$$

onde

$$
\xi_{1}=f_{2}+(1+\omega) f_{1} \quad \text { e } \quad \xi_{2}=\frac{1}{2+\nu}\left(f_{4}+(1+\nu) f_{3}+f_{1}\right)-f_{3} .
$$

Novamente pela continuidade da aplicação traço, podemos concluir que $\chi$ é um funcional linear e contínuo em $H^{1}(\Omega)$, isto é, $\chi \in\left(H^{1}(\Omega)\right)^{\prime}$. Assim pelo teorema de Lax-Milgran existe $z_{1} \in H^{1}(\Omega)$ tal que

$$
\left(\left(\left(z_{1}, v\right)\right)\right)=\langle\chi, v\rangle, \quad \forall v \in H^{1}(\Omega)
$$

Logo,

$$
\left(\nabla z_{1}, \nabla v\right)_{L^{2}(\Omega)}+(2+\omega)\left(z_{1}, v\right)_{L^{2}(\Omega)}+\frac{1}{2+\nu}\left(z_{1}, v\right)_{L^{2}(\Gamma)}=\left(\xi_{1}, v\right)_{L^{2}(\Omega)}+\left(\xi_{2}, v\right)_{L^{2}(\Gamma)} .
$$


Em particular, para $v \in \mathcal{D}(\Omega)$, espaço das funções testes, concluímos que

$$
-\Delta z_{1}+(2+\omega) z_{1}=\xi_{1} \quad \text { em } \quad \mathcal{D}^{\prime}(\Omega)
$$

consequentemente, $-\Delta z_{1} \in L^{2}(\Omega)$ e

$$
-\Delta z_{1}+(2+\omega) z_{1}=f_{2}+(1+\omega) f_{1} \quad \text { em } \quad L^{2}(\Omega) .
$$

Assim, fazendo

$$
z_{2}=z_{1}-f_{1}
$$

obtemos que

$$
z_{1}-\Delta z_{1}+z_{1}+\omega z_{2}=f_{2}
$$

Por outro lado, de (3.14) e usando (3.15) vem que

$$
\begin{aligned}
\left(\nabla z_{1}, \nabla v\right)_{L^{2}(\Omega)}+ & (2+\omega)\left(z_{1}, v\right)_{L^{2}(\Omega)}+\frac{1}{2+\nu}\left(z_{1}, v\right)_{L^{2}(\Gamma)} \\
= & \left(-\Delta z_{1}+(2+\omega) z_{1}, v\right)_{L^{2}(\Omega)}+\left(\xi_{2}, v\right)_{L^{2}(\Gamma)} .
\end{aligned}
$$

Assim,

$$
\begin{aligned}
& \left(\nabla z_{1}, \nabla v\right)_{L^{2}(\Omega)}+\left(\Delta z_{1}, v\right)_{L^{2}(\Omega)} \\
& \quad=\frac{1}{2+\nu}\left(-z_{1}+f_{4}+(1+\nu) f_{3}+f_{1}-(2+\nu) f_{3}, v\right)_{L^{2}(\Gamma)}
\end{aligned}
$$

para todo $v \in H^{1}(\Omega)$. Escolhendo

$$
z_{4}=\frac{1}{2+\nu}\left(-z_{1}+f_{4}+(1+\nu) f_{3}+f_{1}\right)-f_{3} \quad \text { em } \quad L^{2}(\Gamma),
$$

e

$$
z_{3}=z_{4}+f_{3},
$$

obtemos que, $\partial_{\mathbf{n}} z_{1}=z_{4}$ no sentido fraco e que, substituindo (3.18) em (3.19), lembrando que $z_{2}=z_{1}-f_{1}$ e $z_{4}=z_{3}-f_{3}$, tem-se

$$
z_{4}+z_{2}+z_{3}+\nu z_{4}=f_{4}
$$

Portanto, combinando (3.16), (3.17), (3.19) e (3.20) concluímos que dado qualquer $\left(f_{1}, f_{2}, f_{3}, f_{4}\right) \in \mathcal{H}$ existe $\left(z_{1}, z_{2}, z_{3}, z_{4}\right) \in D(A)$ tal que

$$
(I-A)\left(z_{1}, z_{2}, z_{3}, z_{4}\right)^{\top}=\left(f_{1}, f_{2}, f_{3}, f_{4}\right)^{\top}
$$


Etapa 4: $\mathcal{F}: \mathcal{H} \rightarrow \mathcal{H}$ é localmente Lipschitz.

Observe que a hipótese (3.8) implica que $\mathcal{F}: \mathcal{H} \rightarrow \mathcal{H}$ é localmente Lipschitz. De fato, basta notar que vale a seguinte estimativa:

$$
\begin{aligned}
\left\|\mathcal{F}\left(z_{1}, z_{2}, z_{3}, z_{4}\right)-\mathcal{F}\left(\bar{z}_{1}, \bar{z}_{2}, \bar{z}_{3}, \bar{z}_{4}\right)\right\|_{\mathcal{H}}^{2} & =\int_{\Omega}\left|f\left(z_{1}\right)-f\left(\bar{z}_{1}\right)\right|^{2} d x \\
& \leq \int_{\Omega}\left|f^{\prime}\left(\theta z_{1}+(1-\theta) \bar{z}_{1}\right)\right|^{2}\left(z_{1}-\bar{z}_{1}\right)^{2} d x .
\end{aligned}
$$

Logo, da estimativa (3.8), obtemos que $\mathcal{F}$ é Lipschitziana em qualquer limitado de $\mathcal{H}$.

Etapa 5: Conclusão, está garantida a existência e unicidade local de soluções generalizadas.

Das Etapas 2,3 e 4 obtemos que o operador $A$ é maximal e monótono e $\mathcal{F}$ é localmente lipschitz. Além disso $\overline{D(A)}=\mathcal{H}$. Então dado o par $\tau \leq t$ e dado uma condição inicial $z_{\tau} \in \mathcal{H}$, como $h \in L_{\text {loc }}^{2}\left(\mathbb{R} ; L^{2}(\Omega)\right)$, pelo Teorema 1.0.15, o problema de Cauchy (3.12) tem uma única solução generalizada (limite de solução forte) local $z \in C^{0}\left(\left[\tau, t_{\max }\right) ; \mathcal{H}\right)$. Mais ainda, analogamente, para cada $\tau \in \mathbb{R}$ se $z_{\tau} \in D(A)$ e desde que $h \in H_{\text {loc }}^{1}\left(\mathbb{R} ; L^{2}(\Omega)\right)$, então o problema tem uma solução forte local definida em $\left[\tau, t_{\max }\right)$ na classe

$$
z \in C^{1}\left(\left[\tau, t_{\max }\right), D(A)\right) \cap C^{0}\left(\left[\tau, t_{\max }\right), \mathcal{H}\right) .
$$

Em ambos os casos se

$$
t_{\max }<\infty \quad \text { então } \quad \lim _{t \rightarrow t_{\max }}\|z(t)\|_{\mathcal{H}}=\infty
$$

Assim, se $\|z(t)\|_{\mathcal{H}}<\infty$, então necessariamente $t_{\max }=\infty$, isto é, $z$ é uma solução global em ambos os casos.

Etapa 6: $t_{\max }=\infty$.

Mostremos que, de fato, $\|z(t)\|_{\mathcal{H}}<\infty$. Observe que,

$$
\begin{aligned}
\frac{1}{2} \frac{d}{d t}\left\{\left\|\left(u, u_{t}, \delta, \delta_{t}\right)\right\|_{\mathcal{H}}^{2}\right. & \left.+2 \int_{\Omega} F(u)\right\}=-\omega\left\|u_{t}\right\|_{L^{2}(\Omega)}^{2}-\nu\left\|\delta_{t}\right\|_{L^{2}(\Gamma)}^{2}+\int_{\Omega} h u_{t} \\
& \leq(-\omega+\varepsilon)\left\|u_{t}\right\|_{L^{2}(\Omega)}^{2}-\nu\left\|\delta_{t}\right\|_{L^{2}(\Gamma)}^{2}+C(\varepsilon)\|h(t)\|_{L^{2}(\Omega)}^{2} \\
& \leq C(\varepsilon)\|h(t)\|_{L^{2}(\Omega)}^{2} .
\end{aligned}
$$

Integrando de $\tau$ a $t$, obtemos

$$
\begin{aligned}
\frac{1}{2}\left\|\left(u(t), u_{t}(t), \delta(t), \delta_{t}(t)\right)\right\|_{\mathcal{H}}^{2}+ & \int_{\Omega} F(u(t)) \leq \frac{1}{2}\left\|\left(u(\tau), u_{t}(\tau), \delta(\tau), \delta_{t}(\tau)\right)\right\|_{\mathcal{H}}^{2} \\
& +\int_{\Omega} F(u(\tau))+C(\varepsilon) \int_{\tau}^{t}\|h(s)\|_{L^{2}(\Omega)}^{2} .
\end{aligned}
$$


Usando que,

$$
F(u) \leq C\left(1+|u|^{4}\right)
$$

e

$$
\int_{\Omega} F(u) \geq-\frac{\beta}{2}\|u\|_{H^{1}(\Omega)}^{2}+C_{f}
$$

Concluimos que

$$
\left\|\left(u, u_{t}, \delta, \delta_{t}\right)\right\|_{\mathcal{H}}^{2} \leq C\left(z_{\tau}\right)+C(\varepsilon) \int_{\tau}^{t}\|h(s)\|_{L^{2}(\Omega)}^{2}
$$

Agora, note que,

$$
\begin{aligned}
C(\varepsilon) \int_{\tau}^{t} e^{\sigma_{0}(s-s)}\|h(s)\|_{L^{2}(\Omega)}^{2} & \leq C(\varepsilon) \sup _{s \in(\tau, t)}\left\{e^{-\sigma_{0} s}\right\} \int_{-\infty}^{t} e^{\sigma_{0} s}\|h(s)\|_{L^{2}(\Omega)}^{2}, \\
& \leq C(\varepsilon) C(\tau) \int_{-\infty}^{t} e^{\sigma_{0} s}\|h(s)\|_{L^{2}(\Omega)}^{2},
\end{aligned}
$$

onde

$$
C(\tau)=\sup _{s \in(\tau, t)}\left\{e^{-\sigma_{0} s}\right\}=e^{-\sigma_{0} \tau},
$$

é uma constante que depende do $\tau$.

Note que $\tau$ está fixado e que pela hipótese (3.10),

$$
\int_{-\infty}^{t} e^{\sigma_{0} s}\|h(s)\|_{L^{2}(\Omega)}^{2}<\infty .
$$

Portanto

$$
\left\|\left(u(t), u_{t}(t), \delta(t), \delta_{t}(t)\right)\right\|_{\mathcal{H}}^{2} \leq C\left(z_{\tau}\right)+C(\varepsilon) \int_{\tau}^{t}\|h(s)\|_{L^{2}(\Omega)}^{2} \leq C\left(z_{\tau}, \tau\right) .
$$

Note que esta desigualdade vale para soluções regulares e, também, para soluções generalizadas por densidade. Portanto, se $t_{\max }<\infty$ teríamos uma contradição com a limitação acima. Isto prova que $t_{\max }=\infty$.

Etapa 7: Finalmente vamos provar a desigualdade (3.11).

Dados $T \geq \tau$, seja $z^{1}=\left(u^{1}(t), u_{t}^{1}(t), \delta^{1}(t), \delta_{t}^{1}(t)\right)$ e $z^{2}=\left(u^{2}(t), u_{t}^{2}(t), \delta^{2}(t), \delta_{t}^{2}(t)\right)$ duas soluções de (3.3)-(3.7) cujos respectivos dados iniciais são $z^{1}(\tau)=z_{\tau}^{1}$ e $z^{2}(\tau)=z_{\tau}^{2}$. Assim, definindo $w=u^{1}-u^{2}$ e $\zeta=\delta^{1}-\delta^{2}$, temos que

$$
\begin{aligned}
& w_{t t}-\Delta w+w+\omega w_{t}=f\left(u^{2}\right)-f\left(u^{1}\right), \quad x \in \Omega, \quad t \geq \tau, \\
& \zeta_{t t}+\nu \zeta_{t}+\zeta=-w_{t}, \quad x \in \Gamma, \quad t \geq \tau, \\
& \zeta_{t}=\partial_{n} w, \quad x \in \Gamma, \quad t \geq \tau,
\end{aligned}
$$


com condições iniciais

$$
\begin{aligned}
& w(x, \tau)=u^{1}(x, \tau)-u^{2}(x, \tau),\left.\quad \partial_{t} w(x, t)\right|_{t=\tau}=u_{t}^{1}(x, \tau)-u_{t}^{2}(x, \tau), \quad x \in \Omega, \\
& \zeta(x, \tau)=\delta^{1}(x, \tau)-\delta^{2}(x, \tau),\left.\quad \partial_{t} \zeta(x, t)\right|_{t=\tau}=\delta_{t}^{1}(x, \tau)-\delta_{t}^{2}(x, \tau), \quad x \in \Gamma .
\end{aligned}
$$

Como anteriormente, multiplicando a primeira equação por $w_{t}$ e a segunda por $\zeta_{t}$, integrando em $\Omega$, obtemos que

$$
\begin{aligned}
\frac{1}{2} \frac{d}{d t}\left\|\left(w(t), w_{t}(t), \zeta(t), \zeta_{t}(t)\right)\right\|_{\mathcal{H}}^{2} \leq & -\omega\left\|w_{t}(t)\right\|_{L^{2}(\Omega)}^{2}-\nu\left\|\zeta_{t}(t)\right\|_{L^{2}(\Gamma)}^{2} \\
& +\int_{\Omega}\left[f\left(u^{2}(t)\right)-f\left(u^{1}(t)\right)\right] w_{t}(t) d x \\
\leq & C\left(z_{\tau}^{1}, z_{\tau}^{2}, \tau\right)\left(\|w(t)\|_{\mathcal{H}}^{2}+\left\|w_{t}(t)\right\|_{\mathcal{H}}^{2}\right) .
\end{aligned}
$$

Pelo lema de Gronwall,

$$
\left\|z^{1}(t)-z^{2}(t)\right\|_{\mathcal{H}}^{2} \leq e^{C\left(z_{\tau}^{1}, z_{\tau}^{2}, \tau\right)(T-\tau)}\left\|z^{1}(\tau)-z^{2}(\tau)\right\|_{\mathcal{H}}^{2}
$$

para todo $t \in[\tau, T]$.

Etapa 8: Regularidade

Análogo ao trabalho do Frigeri [21]. Seja $z_{\tau}=\left(u_{\tau}^{0}, u_{\tau}^{1}, \delta_{\tau}^{0}, \delta_{\tau}^{1}\right) \in \mathcal{H}_{1}$ e considere o seguinte problema de valor inicial em $K=H^{\frac{1}{2}}(\Gamma) \times H^{\frac{1}{2}}(\Gamma)$

$$
\begin{aligned}
& \hat{z}_{t}=B \hat{z}+y, \\
& \hat{z}(\tau)=\left(\delta_{\tau}^{0}, \delta_{\tau}^{1}\right)^{\top},
\end{aligned}
$$

onde $\hat{z}(t)=\left(\hat{z}_{3}(t), \hat{z}_{4}(t)\right)^{\top} \mathrm{e}$

$$
B=\left[\begin{array}{cc}
0 & 1 \\
-1 & -\nu
\end{array}\right] \quad, \quad y(t)=\left[\begin{array}{c}
0 \\
-u_{t}(t)
\end{array}\right] .
$$

Aqui $u_{t}$ é a segunda componente da solução global regular $z$ obtida nas etapas anteriores. Note que $y \in C^{0}([\tau, \infty) ; K)$. Como $B \in \mathcal{L}(X)$ e $\hat{z}(\tau) \in K$, temos que o problema (3.21) admite uma única solução $\hat{z} \in C^{1}([\tau, \infty) ; K)$. Pela comparação com o problema (3.12) e pela unicidade, deduzimos que $\hat{z}_{3}=\delta$ e $\hat{z}_{4}=\delta_{t}$ em $[\tau, \infty)$. Portanto, a solução regular deve satisfazer:

$$
z \in C^{1}\left([\tau, \infty), \mathcal{H}_{1}\right) \cap C^{0}([\tau, \infty), \mathcal{H}) .
$$

sempre que o dado inicial $z_{\tau}$ pertence à $\mathcal{H}_{1}$. 
Observação 3.3.4. Aproximando localmente $h \in L_{\text {loc }}^{2}\left(\mathbb{R} ; L^{2}(\Omega)\right)$ por funções do espaço $H_{l o c}^{1}\left(\mathbb{R} ; L^{2}(\Omega)\right)$, podemos ver que as soluções fracas podem ser aproximadas por soluções regulares. Então, por densidade, todas as estimativas de energias a seguir serão válidas para soluções fracas.

Observação 3.3.5. Toda solução generalizada é uma solução fraca, segundo a Definição 3.3.1, e toda solução fraca é única. Mais ainda, pode escrever:

$$
z(t)=e^{A(t-\tau)} z_{\tau}+\int_{\tau}^{t} e^{A(t-s)}(\mathcal{F}(z(s))+\mathcal{G}(s, z(s))) d s
$$

O operador solução do problema (3.3)-(3.7) obtido no Teorema 3.3.3 define um processo de evolução sobre $\mathcal{H}$ da seguinte forma, dados $\tau \in \mathbb{R}$ e $z_{\tau} \in \mathcal{H}$ defina $U(t, \tau)$ : $\mathcal{H} \rightarrow \mathcal{H}$ por

$$
U(t, \tau) z_{\tau}=z(t)=\left(u(t), u_{t}(t), \delta(t), \delta_{t}(t)\right)
$$

ou seja, $U(t, \tau) z_{\tau}$ é a solução do problema (3.3)-(3.7) no tempo $t$ com tempo inicial $\tau$ e dado inicial $z_{\tau}$. Claramente essa família de operadores satisfaz as propriedades de um processo de evolução, basta usar a unicidade de solução generalizada.

Lema 3.3.6. O processo de evolução $U(t, \tau): \mathcal{H} \rightarrow \mathcal{H}$ associado ao problema (3.3)-(3.7) é fechado segundo a Definição 2.3.2.

Demonstração. Suponha que $z_{\tau}^{n} \rightarrow z_{\tau}$ em $\mathcal{H}$ e que $U(t, \tau) z_{\tau}^{n} \rightarrow y$ em $\mathcal{H}$. Então pela dependência contínua dos dados e pela unicidade do limite, concluímos que $y=U(t, \tau) z_{\tau}$, ou seja, o processo $U(t, \tau)$ é fechado.

\subsection{Desigualdades notáveis}

No que segue vamos supor as hipóteses do Teorema 3.3.3.

Nesta seção apresentaremos algumas estimativas da energia associadas ao problema (3.3)-(3.7). Definimos o funcional energia ao longo de uma solução de (3.3)-(3.7) como

$$
\begin{aligned}
\mathcal{E}(t)=\frac{1}{2}\|u(t)\|_{H^{1}(\Omega)}^{2}+ & \frac{1}{2}\left\|u_{t}(t)\right\|_{L^{2}(\Omega)}^{2}+\frac{1}{2}\|\delta(t)\|_{L^{2}(\Gamma)}^{2} \\
& +\frac{1}{2}\left\|\delta_{t}(t)\right\|_{L^{2}(\Gamma)}^{2}+\int_{\Omega} F(u(t)) d x .
\end{aligned}
$$


Então multiplicando (3.3) por $u_{t}$, integrando em $\Omega$ e multiplicando (3.4) por $\delta_{t} \mathrm{e}$ integrando em $\Gamma$ obtemos

$$
\begin{gathered}
\int_{\Omega} u_{t t} u_{t}-\int_{\Omega} u_{t} \Delta u+\omega \int_{\Omega} u_{t} u_{t}+\int_{\Omega} u u_{t}+\int_{\Omega} f(u) u_{t}=\int_{\Omega} h(x, t) u_{t}, \\
\int_{\Gamma} \delta_{t t} \delta_{t}+\nu \int_{\Gamma} \delta_{t} \delta_{t}+\int_{\Gamma} \delta \delta_{t}=-\int_{\Gamma} u_{t} \delta_{t} .
\end{gathered}
$$

Assim, somando as igualdades e usando o fato de que $\partial_{\mathbf{n}} u=\delta_{t}$ num sentido fraco, obtemos que

$$
\begin{aligned}
\int_{\Omega} u_{t t} u_{t}+\int_{\Omega} \nabla u_{t} \nabla u & +\int_{\Omega} u u_{t}+\int_{\Gamma} \delta_{t t} \delta_{t}+\int_{\Gamma} \delta \delta_{t}-\int_{\Gamma} u_{t} \delta_{t}+\int_{\Omega} f(u) u_{t} \\
& =-\omega \int_{\Omega} u_{t} u_{t}-\nu \int_{\Gamma} \delta_{t} \delta_{t}+\int_{\Omega} h(x, t) u_{t}-\int_{\Gamma} u_{t} \delta_{t} .
\end{aligned}
$$

Consequentemente,

$$
\mathcal{E}^{\prime}(t)=-\omega\left\|u_{t}\right\|_{L^{2}(\Omega)}^{2}-\nu\left\|\delta_{t}\right\|_{L^{2}(\Gamma)}^{2}+\int_{\Omega} h u_{t} d x
$$

Lema 3.4.1. Existem $\beta_{0}, C_{f}, C_{F}$ tais que

$$
\mathcal{E}(t) \geq \beta_{0}\left\|\left(u, u_{t}, \delta, \delta_{t}\right)\right\|_{\mathcal{H}}^{2}-C_{f}, \quad t \geq \tau
$$

$e$

$$
\mathcal{E}(t) \leq C_{F}\left(1+\left\|\left(u, u_{t}, \delta, \delta_{t}\right)\right\|_{\mathcal{H}}^{4}\right), \quad t \geq \tau
$$

Demonstração. Observe que, de (3.9),

$$
\begin{aligned}
\int_{\Omega} F(u) d x & \geq-\frac{\beta}{2} \int_{\Omega}|u|^{2}-\int_{\Omega} m_{f} \\
& \geq-\frac{\beta}{2}\|u\|_{H^{1}(\Omega)}^{2}-m_{f}|\Omega|,
\end{aligned}
$$

Logo

$$
\begin{aligned}
\mathcal{E}(t) & =\frac{1}{2}\left\|\left(u, u_{t}, \delta, \delta_{t}\right)\right\|_{\mathcal{H}}^{2}+\int_{\Omega} F(u) \\
& \geq \frac{1}{2}\left\|\left(u, u_{t}, \delta, \delta_{t}\right)\right\|_{\mathcal{H}}^{2}-\frac{\beta}{2}\|u\|_{H^{1}(\Omega)}^{2}-m_{f}|\Omega| \\
& \geq \beta_{0}\left\|\left(u, u_{t}, \delta, \delta_{t}\right)\right\|_{\mathcal{H}}^{2}-C_{f},
\end{aligned}
$$


onde $\beta_{0}=\frac{1-\beta}{2}$ e $C_{f}=m_{f}|\Omega|$. Por outro lado, de (3.8), existe uma constante $C^{\prime}>0$ tal que $F(u) \leq C^{\prime}\left(1+|u|^{4}\right)$. Então, de 3.22 ,

$$
\begin{aligned}
\mathcal{E}(t) & =\frac{1}{2}\left\|\left(u, u_{t}, \delta, \delta_{t}\right)\right\|_{\mathcal{H}}^{2}+\int_{\Omega} F(u) \\
& \leq \frac{1}{2}\left\|\left(u, u_{t}, \delta, \delta_{t}\right)\right\|_{\mathcal{H}}^{2}+C^{\prime} \int_{\Omega}|u|^{4}+C^{\prime}|\Omega| \\
& =\frac{1}{2}\left\|\left(u, u_{t}, \delta, \delta_{t}\right)\right\|_{\mathcal{H}}^{2}+C^{\prime}\|u\|_{L^{4}(\Omega)}^{4}+C^{\prime}|\Omega| \\
& \leq \frac{1}{2}\left\|\left(u, u_{t}, \delta, \delta_{t}\right)\right\|_{\mathcal{H}}^{2}+C^{\prime \prime}\|u\|_{H^{1}(\Omega)}^{4}+C^{\prime}|\Omega| \\
& \leq \frac{1}{2}\left\|\left(u, u_{t}, \delta, \delta_{t}\right)\right\|_{\mathcal{H}}^{2}+C^{\prime \prime}\left(\left\|\left(u, u_{t}, \delta, \delta_{t}\right)\right\|_{\mathcal{H}}\right)^{4}+C^{\prime}|\Omega| .
\end{aligned}
$$

Portanto, existe uma constante $C_{F}>0$ satisfazendo (3.24).

A seguinte estimativa da energia vai garantir a dissipatividade do sistema.

Lema 3.4.2. Existem constantes $\sigma_{1}, C_{\omega}>0$ tal que

$$
\mathcal{E}(t) \leq 3 \mathcal{E}(\tau) e^{-\sigma_{1}(t-\tau)}+2 C_{\omega} \int_{\tau}^{t} e^{-\sigma_{1}(t-s)}\|h(s)\|_{L^{2}(\Omega)}^{2} d s+6 C_{f}, \quad t \geq \tau
$$

Demonstração. Começamos definindo a energia perturbada

$$
\mathcal{E}_{\alpha}(t)=\mathcal{E}(t)+\alpha \Phi(t), \quad \alpha>0,
$$

onde,

$$
\Phi(t)=\int_{\Omega} u(t) u_{t}(t) d x+\int_{\Gamma} \delta(t) \delta_{t}(t) d \gamma+\int_{\Gamma} u(t) \delta(t) d \gamma
$$

Para concluir, dividimos a demonstração em três etapas.

Etapa 1. Existe $\alpha_{0}>0$ tal que

$$
\frac{1}{2} \mathcal{E}(t)-\frac{1}{2} C_{f} \leq \mathcal{E}_{\alpha}(t) \leq \frac{3}{2} \mathcal{E}(t)+\frac{1}{2} C_{f}, \quad \forall \alpha \in\left[0, \alpha_{0}\right]
$$

Com efeito, do teorema do traço (preliminares),

$$
\begin{aligned}
\int_{\Gamma} u \delta d \gamma & \leq\|u\|_{L^{2}(\Gamma)}\|\delta\|_{L^{2}(\Gamma)} \leq c_{\Gamma}\|u\|_{H^{1}(\Omega)}\|\delta\|_{L^{2}(\Gamma)} \\
& \leq \frac{1}{2}\|u\|_{H^{1}(\Omega)}^{2}+\frac{c_{\Gamma}^{2}}{2}\|\delta\|_{L^{2}(\Gamma)}^{2}
\end{aligned}
$$


e usando a estimativa (3.23), obtemos

$$
\begin{aligned}
|\Phi(t)|= & \left|\int_{\Omega} u(t) u_{t}(t) d x+\int_{\Gamma} \delta(t) \delta_{t}(t) d \gamma+\int_{\Gamma} u(t) \delta(t) d \gamma\right| \\
\leq & \frac{1}{2}\|u(t)\|_{L^{2}(\Omega)}^{2}+\frac{1}{2}\left\|u_{t}(t)\right\|_{L^{2}(\Omega)}^{2}+\frac{1}{2}\|\delta(t)\|_{L^{2}(\Gamma)}^{2} \\
& +\frac{1}{2}\left\|\delta_{t}(t)\right\|_{L^{2}(\Gamma)}^{2}+\frac{1}{2}\|u(t)\|_{H^{1}(\Omega)}^{2}+\frac{c_{\Gamma}^{2}}{2}\|\delta(t)\|_{L^{2}(\Gamma)}^{2} \\
\leq & \left(1+c_{\Gamma}^{2}\right)\left(\|u(t)\|_{H^{1}(\Omega)}^{2}+\left\|u_{t}(t)\right\|_{L^{2}(\Omega)}^{2}+\|\delta(t)\|_{L^{2}(\Gamma)}^{2}+\left\|\delta_{t}(t)\right\|_{L^{2}(\Gamma)}^{2}\right) \\
\leq & \left(1+c_{\Gamma}^{2}\right)\left\|\left(u(t), u_{t}(t), \delta(t), \delta_{t}(t)\right)\right\|_{\mathcal{H}}^{2} \\
\leq & \left(1+c_{\Gamma}^{2}\right) \beta_{0}^{-1}\left(\mathcal{E}(t)+C_{f}\right) .
\end{aligned}
$$

Então, escolhendo $\alpha_{0}=\frac{1}{2} \beta_{0}\left(1+c_{\Gamma}^{2}\right)^{-1}$, concluímos que

$$
\alpha|\Phi(t)| \leq \alpha_{0}|\Phi(t)| \leq \alpha_{0} \frac{\left(1+c_{\Gamma}^{2}\right)}{\beta_{0}}\left(\mathcal{E}(t)+C_{f}\right) \leq \frac{1}{2}\left(\mathcal{E}(t)+C_{f}\right)
$$

desde que $\alpha \leq \alpha_{0}$, consequentemente,

$$
\begin{aligned}
& \mathcal{E}_{\alpha}(t)=\mathcal{E}(t)+\alpha \Phi(t) \leq \frac{3}{2} \mathcal{E}(t)+\frac{1}{2} C_{f} \\
& \mathcal{E}_{\alpha}(t)=\mathcal{E}(t)+\alpha \Phi(t) \geq \frac{1}{2} \mathcal{E}(t)-\frac{1}{2} C_{f}
\end{aligned}
$$

para todo $\alpha \in\left[0, \alpha_{0}\right]$. Concluindo a Etapa 1 .

Etapa 2. Podemos estimar $\Phi^{\prime}(t)$ como segue

$$
\begin{aligned}
\Phi^{\prime}(t) \leq-\mathcal{E}(t)+ & \left(\frac{3}{2}+\frac{2 \omega^{2}}{1-\beta}\right)\left\|u_{t}(t)\right\|_{L^{2}(\Omega)}^{2} \\
& +\left(\frac{3+\nu^{2}}{2}+\frac{4 c_{\Gamma}^{2}}{1-\beta}\right)\left\|\delta_{t}(t)\right\|_{L^{2}(\Gamma)}^{2}+\frac{2}{1-\beta}\|h(t)\|_{L^{2}(\Omega)}^{2}+C_{f} .
\end{aligned}
$$

Usando as equações (3.3)-(3.5),

$$
\begin{aligned}
\Phi^{\prime}(t)= & \int_{\Omega} u_{t}(t) u_{t}(t)+\int_{\Omega} u(t) u_{t t}(t)+\int_{\Gamma} \delta_{t}(t) \delta_{t}(t) \\
& +\int_{\Gamma} \delta_{t t}(t) \delta(t)+\int_{\Gamma} \delta_{t}(t) u(t)+\int_{\Gamma} \delta(t) u_{t}(t) \\
= & \left\|u_{t}(t)\right\|_{L^{2}(\Omega)}^{2}+\int_{\Omega} u(t)\left(\Delta u(t)-\omega u_{t}(t)-u(t)-f(u(t))+h(t)\right) \\
& +\left\|\delta_{t}(t)\right\|_{L^{2}(\Gamma)}^{2} \int_{\Gamma}\left(-\nu \delta_{t}(t)-\delta(t)-u_{t}(t)\right) \delta(t)+\int_{\Gamma} \delta_{t}(t) u(t) \int_{\Gamma} \delta(t) u_{t}(t) .
\end{aligned}
$$


Pela fórmula de Green,

$$
\begin{aligned}
\Phi^{\prime}(t)= & \left\|u_{t}(t)\right\|_{L^{2}(\Omega)}^{2}-\|u(t)\|_{H^{1}(\Omega)}^{2}+\int_{\Gamma} u(t) \partial_{\mathbf{n}} u(t)-\omega \int_{\Omega} u(t) u_{t}(t)-\int_{\Omega} f(u) u \\
& +\int_{\Omega} h(t) u(t)+\left\|\delta_{t}(t)\right\|_{L^{2}(\Gamma)}^{2}-\nu \int_{\Gamma} \delta_{t}(t) \delta(t)-\|\delta(t)\|_{L^{2}(\Gamma)}^{2}-\int_{\Gamma} u_{t}(t) \delta(t) \\
& +\int_{\Gamma} \delta_{t}(t) u(t)+\int_{\Gamma} \delta(t) u_{t}(t) .
\end{aligned}
$$

Lembrando que $\partial_{\mathbf{n}} u=\delta_{t}$ em $\Gamma$,

$$
\begin{aligned}
\Phi^{\prime}(t)= & \left\|u_{t}(t)\right\|_{L^{2}(\Omega)}^{2}-\|u(t)\|_{H^{1}(\Omega)}^{2}-\omega \int_{\Omega} u(t) u_{t}(t)-\int_{\Omega} f(u(t)) u(t)+\int_{\Omega} h(t) u(t) \\
& +\left\|\delta_{t}(t)\right\|_{L^{2}(\Gamma)}^{2}-\|\delta(t)\|_{L^{2}(\Gamma)}^{2}-\nu \int_{\Gamma} \delta(t) \delta_{t}(t)+2 \int_{\Gamma} \delta_{t}(t) u(t) .
\end{aligned}
$$

Iremos omitir $t$ para simplificar. Inserindo $\mathcal{E}(t)$, e usando (3.9), obtemos que

$$
\begin{aligned}
\Phi^{\prime}(t)=- & \left.\frac{1}{2}\|u\|_{H^{1}(\Omega)}^{2}+\frac{1}{2}\left\|u_{t}\right\|_{L^{2}(\Omega)}^{2}+\frac{1}{2}\|\delta\|_{L^{2}(\Gamma)}^{2}+\frac{1}{2}\left\|\delta_{t}\right\|_{L^{2}(\Gamma)}^{2}+\int_{\Omega} F(u) d x\right) \\
- & \frac{1}{2}\|u\|_{H^{1}(\Omega)}^{2}-\frac{1}{2}\|\delta\|_{L^{2}(\Gamma)}^{2}+\frac{3}{2}\left\|u_{t}\right\|_{L^{2}(\Omega)}^{2}+\frac{3}{2}\left\|\delta_{t}\right\|_{L^{2}(\Gamma)}^{2}+\int_{\Omega}[F(u)-f(u) u] \\
& +\int_{\Omega} h u-\omega \int_{\Omega} u_{t} u-\nu \int_{\Gamma} \delta_{t} \delta+2 \int_{\Gamma} \delta_{t} u \\
\leq- & \mathcal{E}(t)-\frac{1}{2}\|u\|_{H^{1}(\Omega)}^{2}-\frac{1}{2}\|\delta\|_{L^{2}(\Gamma)}^{2}+\frac{3}{2}\left\|u_{t}\right\|_{L^{2}(\Omega)}^{2}+\frac{3}{2}\left\|\delta_{t}\right\|_{L^{2}(\Gamma)}^{2} \\
& +\int_{\Omega}\left(\frac{\beta}{2} u^{2}+m_{f}\right)+\int_{\Omega} h u-\omega \int_{\Omega} u_{t} u-\nu \int_{\Gamma} \delta_{t} \delta+2 \int_{\Gamma} \delta_{t} u \\
\leq- & \mathcal{E}(t)-\frac{1}{2}\|u\|_{H^{1}(\Omega)}^{2}-\frac{1}{2}\|\delta\|_{L^{2}(\Gamma)}^{2}+\frac{3}{2}\left\|u_{t}\right\|_{L^{2}(\Omega)}^{2}+\frac{3}{2}\left\|\delta_{t}\right\|_{L^{2}(\Gamma)}^{2} \\
& +\frac{\beta}{2}\|u\|_{H^{1}(\Omega)}^{2}+C_{f}+\int_{\Omega} h u-\omega \int_{\Omega} u_{t} u-\nu \int_{\Gamma} \delta_{t} \delta+2 \int_{\Gamma} \delta_{t} u \\
=- & \mathcal{E}(t)-\left(\frac{1-\beta}{2}\right)\|u\|_{H^{1}(\Omega)}^{2}-\frac{1}{2}\|\delta\|_{L^{2}(\Gamma)}^{2}+\frac{3}{2}\left\|u_{t}\right\|_{L^{2}(\Omega)}^{2}+\frac{3}{2}\left\|\delta_{t}\right\|_{L^{2}(\Gamma)}^{2} \\
& +\int_{\Omega} h u-\omega \int_{\Omega} u_{t} u-\nu \int_{\Gamma} \delta_{t} \delta+2 \int_{\Gamma} \delta_{t} u+C_{f} .
\end{aligned}
$$

Note que

$$
\begin{aligned}
&-\omega \int_{\Omega} u u_{t} \leq \omega\|u\|_{L^{2}(\Omega)}\left\|u_{t}\right\|_{L^{2}(\Omega)} \leq \omega\|u\|_{H^{1}(\Omega)}\left\|u_{t}\right\|_{L^{2}(\Omega)} \\
& \leq \frac{1-\beta}{8}\|u\|_{H^{1}(\Omega)}^{2}+\frac{2 \omega^{2}}{1-\beta}\left\|u_{t}\right\|_{L^{2}(\Omega)}^{2}, \\
& \int_{\Omega} h u \leq \frac{1-\beta}{8}\|u\|_{H^{1}(\Omega)}^{2}+\frac{2}{1-\beta}\|h\|_{L^{2}(\Omega)}^{2}, \\
& \nu \int_{\Gamma} \delta \delta_{t} \leq \frac{1}{2}\|\delta\|_{L^{2}(\Gamma)}^{2}+\frac{\nu^{2}}{2}\left\|\delta_{t}\right\|_{L^{2}(\Gamma)}^{2},
\end{aligned}
$$




$$
\begin{aligned}
2 \int_{\Gamma} u \delta_{t} d \gamma & \leq 2\|u\|_{L^{2}(\Gamma)}\left\|\delta_{t}\right\|_{L^{2}(\Gamma)} \leq 2 c_{\Gamma}\|u\|_{H^{1}(\Omega)}\left\|\delta_{t}\right\|_{L^{2}(\Gamma)} \\
& \leq \frac{1-\beta}{4}\|u\|_{H^{1}(\Omega)}^{2}+\frac{4 c_{\Gamma}^{2}}{1-\beta}\left\|\delta_{t}\right\|_{L^{2}(\Gamma)}^{2} .
\end{aligned}
$$

Portanto, voltando em (3.28) e combinando com as estimativas acima, temos que

$$
\begin{aligned}
\Phi^{\prime}(t) \leq- & \mathcal{E}(t)-\left(\frac{1-\beta}{2}\right)\|u\|_{H^{1}(\Omega)}^{2}-\frac{1}{2}\|\delta\|_{L^{2}(\Gamma)}^{2}+\frac{3}{2}\left\|u_{t}\right\|_{L^{2}(\Omega)}^{2}+\frac{3}{2}\left\|\delta_{t}\right\|_{L^{2}(\Gamma)}^{2} \\
& +\int_{\Omega} h u-\omega \int_{\Omega} u_{t} u-\nu \int_{\Gamma} \delta_{t} \delta+2 \int_{\Gamma} \delta_{t} u+C_{f} \\
\leq- & \mathcal{E}(t)-\left(\frac{1-\beta}{2}\right)\|u\|_{H^{1}(\Omega)}^{2}-\frac{1}{2}\|\delta\|_{L^{2}(\Gamma)}^{2}+\frac{3}{2}\left\|u_{t}\right\|_{L^{2}(\Omega)}^{2}+\frac{3}{2}\left\|\delta_{t}\right\|_{L^{2}(\Gamma)}^{2} \\
& +\left(\frac{1-\beta}{8}\right)\|u\|_{H^{1}(\Omega)}^{2}+\left(\frac{2}{1-\beta}\right)\|h\|_{L^{2}(\Omega)}^{2}+\left(\frac{1-\beta}{8}\right)\|u\|_{H^{1}(\Omega)}^{2} \\
& +\left(\frac{2 \omega^{2}}{1-\beta}\right)\left\|u_{t}\right\|_{L^{2}(\Omega)}^{2}+\frac{1}{2}\|\delta\|_{L^{2}(\Gamma)}^{2}+\frac{\nu^{2}}{2}\left\|\delta_{t}\right\|_{L^{2}(\Gamma)}^{2}+\left(\frac{1-\beta}{4}\right)\|u\|_{H^{1}(\Omega)}^{2} \\
& \quad+\left(\frac{4 c_{\Gamma}^{2}}{1-\beta}\right)\left\|\delta_{t}\right\|_{L^{2}(\Gamma)}^{2} \\
=- & \mathcal{E}(t)+\left(\frac{3}{2}+\frac{2 \omega^{2}}{1-\beta}\right)\left\|u_{t}(t)\right\|_{L^{2}(\Omega)}^{2}+\left(\frac{3+\nu^{2}}{2}+\frac{4 c_{\Gamma}^{2}}{1-\beta}\right)\left\|\delta_{t}(t)\right\|_{L^{2}(\Gamma)}^{2} \\
+ & \left(\frac{2}{1-\beta}\right)\|h(t)\|_{L^{2}(\Omega)}^{2}+C_{f} .
\end{aligned}
$$

Isso conclui a Etapa 2.

Etapa 3. Conclusão: Levando em conta que

$$
\begin{aligned}
\mathcal{E}^{\prime}(t) & \leq-\omega\left\|u_{t}\right\|_{L^{2}(\Omega)}^{2}-\nu\left\|\delta_{t}\right\|_{L^{2}(\Gamma)}^{2}+\int_{\Omega} h u \\
& \leq-\frac{\omega}{2}\left\|u_{t}\right\|_{L^{2}(\Omega)}^{2}-\nu\left\|\delta_{t}\right\|_{L^{2}(\Gamma)}^{2}+\frac{1}{2 \omega}\|h(t)\|_{L^{2}(\Omega)}^{2}
\end{aligned}
$$

escolhemos

$$
\alpha=\min \left\{\alpha_{0}, \frac{\omega}{2}\left(\frac{3}{2}+\frac{2 \omega^{2}}{1-\beta}\right)^{-1}, \nu\left(\frac{3+\nu^{2}}{2}+\frac{4 c_{\Gamma}^{2}}{1-\beta}\right)^{-1}\right\} .
$$

Assim

$$
\begin{aligned}
\mathcal{E}_{\alpha}^{\prime}(t)=\mathcal{E}^{\prime}(t) & +\alpha \Phi^{\prime}(t) \\
\leq- & \frac{\omega}{2}\left\|u_{t}\right\|_{L^{2}(\Omega)}^{2}-\nu\left\|\delta_{t}\right\|_{L^{2}(\Gamma)}^{2}+\frac{1}{2 \omega}\|h\|_{L^{2}(\Omega)}^{2}-\alpha \mathcal{E}(t) \\
+ & \alpha\left(\frac{3}{2}+\frac{2 \omega^{2}}{1-\beta}\right)\left\|u_{t}(t)\right\|_{L^{2}(\Omega)}^{2}+\alpha\left(\frac{3+\nu^{2}}{2}+\frac{4 c_{\Gamma}^{2}}{1-\beta}\right)\left\|\delta_{t}(t)\right\|_{L^{2}(\Gamma)}^{2} \\
& +\alpha\left(\frac{2}{1-\beta}\right)\|h(t)\|_{L^{2}(\Omega)}^{2}+\alpha C_{f} .
\end{aligned}
$$


Como $\alpha \leq \alpha_{0} \leq \frac{\beta_{0}}{2} \leq \frac{1-\frac{\beta}{2}}{2}=\frac{1-\beta}{4}$, tem-se

$$
\begin{aligned}
\mathcal{E}_{\alpha}^{\prime}(t) \leq- & \frac{\omega}{2}\left\|u_{t}\right\|_{L^{2}(\Omega)}^{2}-\nu\left\|\delta_{t}\right\|_{L^{2}(\Gamma)}^{2}+\frac{1}{2 \omega}\|h\|_{L^{2}(\Omega)}^{2}-\alpha \mathcal{E}(t) \\
& +\frac{\omega}{2}\left\|u_{t}\right\|_{L^{2}(\Omega)}^{2}+\nu\left\|\delta_{t}\right\|_{L^{2}(\Gamma)}^{2}+\frac{1}{2}\|h\|_{L^{2}(\Omega)}^{2}+\alpha C_{f} \\
\leq & -\alpha \mathcal{E}(t)+\left(\frac{1}{2}+\frac{1}{2 \omega}\right)\|h(t)\|_{L^{2}(\Omega)}^{2}+\alpha C_{f} .
\end{aligned}
$$

Defina $C_{\omega}=\frac{1}{2}+\frac{1}{2 \omega}$. Assim, usando (3.26) podemos ver que

$$
\begin{aligned}
\mathcal{E}_{\alpha}^{\prime}(t) & \leq-\frac{2 \alpha}{3} \mathcal{E}_{\alpha}(t)+\frac{\alpha}{3} C_{f}+C_{\Omega}\|h\|_{L^{2}(\omega)}^{2}+\alpha C_{f} \\
& \leq-\frac{2 \alpha}{3} \mathcal{E}_{\alpha}(t)+C_{\omega}\|h(t)\|_{L^{2}(\Omega)}^{2}+\frac{4 \alpha}{3} C_{f}
\end{aligned}
$$

e pela desigualdade de Gronwall (preliminares),

$$
\begin{aligned}
\mathcal{E}_{\alpha}(t) & \leq \mathcal{E}_{\alpha}(\tau) e^{-\frac{2 \alpha}{3}(t-\tau)}+C_{\omega} \int_{\tau}^{t} e^{-\frac{2 \alpha}{3}(t-s)}\|h(s)\|_{L^{2}(\Omega)}^{2} d s+\frac{4 \alpha}{3} C_{f} \int_{\tau}^{t} e^{-\frac{2 \alpha}{3}(t-s)} d s \\
& \leq \mathcal{E}_{\alpha}(\tau) e^{-\frac{2 \alpha}{3}(t-\tau)}+C_{\omega} \int_{\tau}^{t} e^{-\frac{2 \alpha}{3}(t-s)}\|h(s)\|_{L^{2}(\Omega)}^{2} d s+2 C_{f} .
\end{aligned}
$$

Usando novamente (3.26), e definindo $\sigma_{1}=2 \alpha / 3$, obtemos que

$$
\begin{aligned}
\mathcal{E}(t) & \leq 2 \mathcal{E}_{\alpha}(t)+C_{f} \\
& \leq 2 \mathcal{E}_{\alpha}(\tau) e^{-\sigma_{1}(t-\tau)}+2 C_{\omega} \int_{\tau}^{t} e^{-\sigma_{1}(t-s)}\|h(s)\|_{L^{2}(\Omega)}^{2} d s+4 C_{f}+C_{f} \\
& \leq 2\left(\frac{3}{2} \mathcal{E}(\tau)+\frac{1}{2} C_{f}\right) e^{-\sigma_{1}(t-\tau)}+2 C_{\omega} \int_{\tau}^{t} e^{-\sigma_{1}(t-s)}\|h(s)\|_{L^{2}(\Omega)}^{2} d s+5 C_{f} \\
& \leq 3 \mathcal{E}(\tau) e^{-\sigma_{1}(t-\tau)}+2 C_{\omega} \int_{\tau}^{t} e^{-\sigma_{1}(t-s)}\|h(s)\|_{L^{2}(\Omega)}^{2} d s+6 C_{f} .
\end{aligned}
$$

Portanto, segue (3.25).

Observação 3.4.3. Combinando (3.25) com (3.23)-(3.24), existem $C_{1}, C_{2}, C_{3}>0$ tais que, para qualquer $z \in \mathcal{H}$,

$$
\|U(t, \tau) z\|_{\mathcal{H}}^{2} \leq C_{1}\left(1+\|z\|_{\mathcal{H}}^{4}\right) e^{-\sigma_{1}(t-\tau)}+C_{2} \int_{\tau}^{t} e^{-\sigma_{1}(t-s)}\|h(s)\|_{L^{2}(\Omega)}^{2} d s+C_{3} C_{f},
$$

para todo par $\tau \leq t$.

A próxima estimativa vai comparar duas soluções do problema (3.3)-(3.7) afim de auxiliar na demonstração da compacidade assintótica. 
Lema 3.4.4. Dados quaisquer $z_{\tau}^{1}, z_{\tau}^{2} \in \mathcal{H}$. Temos que existe $\sigma_{2}>\sigma_{1}$, tal que

$$
\begin{aligned}
\left\|U(t, \tau) z_{\tau}^{1}-U(t, \tau) z_{\tau}^{2}\right\|_{\mathcal{H}}^{2} \leq & 3\left\|z_{\tau}^{1}-z_{\tau}^{2}\right\|_{\mathcal{H}}^{2} e^{-\sigma_{2}(t-\tau)}+C_{t, \tau} \int_{\tau}^{t}\left\|u^{1}(s)-u^{2}(s)\right\|_{L^{4}(\Omega)}^{2} d s \\
& +4 \int_{\tau}^{t} \int_{\Omega}\left(f\left(u^{2}\right)-f\left(u^{1}\right)\right)\left(u_{t}^{1}-u_{t}^{2}\right) d x d s
\end{aligned}
$$

onde $U(t, \tau) z_{\tau}^{i}=\left(u^{i}(t), \partial_{t} u^{i}(t), \delta^{i}(t), \partial_{t} \delta^{i}(t)\right)$ e $C_{t, \tau}>0$ é uma constante.

Demonstração. A prova é dividida em três etapas.

Etapa 1. Denote $w=u^{1}-u^{2}$ e $\zeta=\delta^{1}-\delta^{2}$. Então $\xi, \zeta$ satisfazem

$$
\begin{aligned}
& w_{t t}-\Delta w+w+\omega w_{t}=f\left(u^{2}\right)-f\left(u^{1}\right), \quad x \in \Omega, \quad t \geq \tau \\
& \zeta_{t t}+\nu \zeta_{t}+\zeta=-w_{t}, \quad x \in \Gamma, \quad t \geq \tau, \\
& \zeta_{t}=\partial_{n} w, \quad x \in \Gamma, \quad t \geq \tau,
\end{aligned}
$$

com condições iniciais

$$
\begin{array}{ll}
\xi(x, \tau)=u^{1}(x, \tau)-u^{2}(x, \tau), & \left.\partial_{t} \xi(x, t)\right|_{t=\tau}=u_{t}^{1}(x, \tau)-u_{t}^{2}(x, \tau), \quad x \in \Omega, \\
\zeta(x, \tau)=\delta^{1}(x, \tau)-\delta^{2}(x, \tau), & \left.\partial_{t} \zeta(x, t)\right|_{t=\tau}=\delta_{t}^{1}(x, \tau)-\delta_{t}^{2}(x, \tau), \quad x \in \Gamma .
\end{array}
$$

Defina o seguinte funcional

$$
G(t)=\frac{1}{2}\left\|\left(w(t), w_{t}(t), \zeta(t), \zeta_{t}(t)\right)\right\|_{\mathcal{H}}^{2},
$$

para todo $t \geq \tau$. Então, multiplicando (3.32) por $w_{t}$, multiplicando (3.33) por $\zeta_{t}$ e usando (3.34), deduzimos que

$$
G^{\prime}(t)=-\omega\left\|w_{t}\right\|_{L^{2}(\Omega)}^{2}-\nu\left\|\zeta_{t}\right\|_{L^{2}(\Gamma)}^{2}+\int_{\Omega}\left(f\left(u^{2}\right)-f\left(u^{1}\right)\right) w_{t} d x
$$

Etapa 2. Agora, definimos a energia perturbada por

$$
G_{\eta}(t)=G(t)+\eta \Psi(t), \eta \geq 0
$$

onde

$$
\Psi(t)=\int_{\Omega} w(t) w_{t}(t) d x+\int_{\Gamma} \zeta(t) \zeta_{t}(t) d \gamma+\int_{\Gamma} w(t) \zeta(t) d \gamma
$$


Note que,

$$
\begin{aligned}
|\Psi(t)| & \leq\left|\int_{\Omega} w(t) w_{t}(t)\right|+\left|\int_{\Gamma} \zeta(t) \zeta_{t}(t)\right|+\left|\int_{\Gamma} w(t) \zeta(t)\right| \\
& \leq\|w\|_{L^{2}(\Omega)}\left\|w_{t}\right\|_{L^{2}(\Omega)}+\|\zeta\|_{L^{2}(\Gamma)}\left\|\zeta_{t}\right\|_{L^{2}(\Gamma)}+\|w\|_{L^{2}(\Gamma)}\|\zeta\|_{L^{2}(\Gamma)} \\
& \leq \frac{1}{2}\|w\|_{L^{2}(\Omega)}^{2}+\frac{1}{2}\left\|w_{t}\right\|_{L^{2}(\Omega)}^{2}+\frac{1}{2}\|\zeta\|_{L^{2}(\Gamma)}^{2}+\frac{1}{2}\left\|\zeta_{t}\right\|_{L^{2}(\Gamma)}^{2}+c_{\Gamma}\|w\|_{H^{1}(\Omega)}\|\zeta\|_{L^{2}(\Gamma)} \\
& \leq \frac{1}{2}\|w\|_{L^{2}(\Omega)}^{2}+\frac{1}{2}\left\|w_{t}\right\|_{L^{2}(\Omega)}^{2}+\frac{1}{2}\|\zeta\|_{L^{2}(\Gamma)}^{2}+\frac{1}{2}\left\|\zeta_{t}\right\|_{L^{2}(\Gamma)}^{2}+\frac{c_{\Gamma}^{2}}{2}\|w\|_{H^{1}(\Omega)}^{2}+\frac{1}{2}\|\zeta\|_{L^{2}(\Gamma)}^{2} \\
& \leq\left(1+c_{\Gamma}^{2}\right) G(t) .
\end{aligned}
$$

Assim, desde que $\eta<\eta_{0}=\frac{1}{2\left(1+c_{\Gamma}^{2}\right)}$,

$$
G_{\eta}(t)=G(t)+\eta \Psi(t) \leq G(t)+\eta\left(1+c_{\Gamma}^{2}\right) G(t) \leq \frac{3}{2} G(t)
$$

para todo $t \geq \tau$, e

$$
G_{\eta}(t) \geq G(t)-\eta\left(1+c_{\Gamma}^{2}\right) G(t) \geq \frac{1}{2} G(t)
$$

para todo $t \geq \tau$. Isto é, sempre que $\eta \in\left[0, \eta_{0}\right]$,

$$
\frac{1}{2} G(t) \leq G_{\eta}(t) \leq \frac{3}{2} G(t)
$$

para todo $t \geq \tau$.

Etapa 3. Procedendo como na prova do Lema 3.4.2 (Lema anterior), podemos ver que

$$
\begin{aligned}
\Psi^{\prime}(t) \leq- & G(t)+\left(\frac{3}{2}+\omega^{2}\right)\left\|w_{t}\right\|_{L^{2}(\Omega)}^{2}+\left(\frac{3+\nu^{2}}{2}+4 c_{\Gamma}^{2}\right)\left\|\zeta_{t}\right\|_{L^{2}(\Gamma)}^{2} \\
& +\int_{\Omega}\left(f\left(u^{2}\right)-f\left(u^{1}\right)\right) w d x
\end{aligned}
$$


De fato,

$$
\begin{aligned}
& \Psi^{\prime}(t) \leq \int_{\Omega} w_{t t} w+\left\|w_{t}\right\|_{L^{2}(\Omega)}^{2}+\int_{\Gamma} \zeta_{t t} \zeta+\left\|\zeta_{t}\right\|_{L^{2}(\Gamma)}^{2}+\int_{\Gamma} w_{t} \zeta+\int_{\Gamma} \zeta_{t} w \\
& \leq \int_{\Omega} w\left(\Delta w-w-\omega w_{t}+f\left(u^{2}\right)-f\left(u^{1}\right)\right)+\left\|w_{t}\right\|_{L^{2}(\Omega)}^{2} \\
& \quad+\int_{\Gamma} \zeta\left(-\nu \zeta_{t}-\zeta-w_{t}\right)+\left\|\zeta_{t}\right\|_{L^{2}(\Gamma)}^{2}+\int_{\Gamma} w_{t} \zeta+\int_{\Gamma} \zeta_{t} w \\
& \leq\|w\|_{H^{1}(\Omega)}^{2}-\omega \int_{\Omega} w_{t} w+\int_{\Omega}\left(f\left(u^{2}\right)-f\left(u^{1}\right)\right) w+\left\|w_{t}\right\|_{L^{2}(\Omega)}^{2} \\
& \quad-\|\zeta\|_{L^{2}(\Gamma)}^{2}-\nu \int_{\Gamma} \zeta \zeta_{t}+\left\|\zeta_{t}\right\|_{L^{2}(\Gamma)}^{2}+2 \int_{\Gamma} \zeta_{t} w \\
& \leq-\|w\|_{H^{1}(\Omega)}^{2}-\|\zeta\|_{L^{2}(\Gamma)}^{2}+\frac{1}{4}\|w\|_{H^{1}(\Omega)}^{2}+\omega^{2}\left\|w_{t}\right\|_{L^{2}(\Omega)}^{2}+\left\|w_{t}\right\|_{L^{2}(\Omega)}^{2}+\left\|\zeta_{t}\right\|_{L^{2}(\Gamma)}^{2} \\
&+\frac{1}{2}\|\zeta\|_{L^{2}(\Gamma)}^{2}+\frac{\nu^{2}}{2}\left\|\zeta_{t}\right\|_{L^{2}(\Gamma)}^{2}+\frac{1}{4}\|w\|_{H^{1}(\Omega)}^{2}+4 c_{\Gamma}^{2}\left\|\zeta_{t}\right\|_{L^{2}(\Gamma)}^{2} \\
& \quad+\int_{\Omega}\left(f\left(u^{2}\right)-f\left(u^{1}\right)\right) w \\
& \leq-G(t)+\left(\frac{3}{2}+\omega^{2}\right)\left\|w_{t}\right\|_{L^{2}(\Omega)}^{2}+\left(\frac{3+\nu^{2}}{2}+4 c_{\Gamma}^{2}\right)\left\|\zeta_{t}\right\|_{L^{2}(\Gamma)}^{2} \\
& \quad+\int_{\Omega}\left(f\left(u^{2}\right)-f\left(u^{1}\right)\right) w .
\end{aligned}
$$

Etapa 4. Em vista de (3.35), escolhendo

$$
\eta=\min \left\{\eta_{0}, \omega\left(\frac{3}{2}+\omega^{2}\right)^{-1}, \nu\left(\frac{3+\nu^{2}}{2}+4 c_{\Gamma}^{2}\right)^{-1}\right\}
$$

Concluímos que

$$
\begin{aligned}
G_{\eta}(t) \leq & G_{\eta}(\tau) e^{-\frac{2 \eta}{3}(t-\tau)}+\sup _{s \in[\tau, t]} k(\tau, s) \int_{\tau}^{t} e^{-\frac{2 \eta}{3}(t-s)}\|w(s)\|_{L^{4}(\Omega)}^{2} d s \\
& +\int_{\tau}^{t} e^{-\frac{2 \eta}{3}(t-s)} \int_{\Omega}\left(f\left(u^{2}(s)\right)-f\left(u^{1}(s)\right)\right) w_{t}(s) d x d s
\end{aligned}
$$

onde $k(\tau, t)=C^{\prime \prime}\left(1+\left\|U(t, \tau) z_{\tau}^{1}\right\|_{\mathcal{H}}^{2}+\left\|U(t, \tau) z_{\tau}^{2}\right\|_{\mathcal{H}}^{2}\right)$, para alguma constante positiva $C^{\prime \prime}>0$. De fato, observe que,

$$
\begin{aligned}
G_{\eta}^{\prime}(t) \leq-\eta G(t)+\int_{\Omega} & \left(f\left(u^{2}(t)\right)-f\left(u^{1}(t)\right)\right) w(t) d x \\
+ & \int_{\Omega}\left(f\left(u^{2}(t)\right)-f\left(u^{1}(t)\right)\right) w_{t}(t) d x .
\end{aligned}
$$


Com efeito, de (3.35) e de (3.37),

$$
\begin{aligned}
G_{\eta}^{\prime}(t)=G^{\prime}( & t)+\eta \Psi^{\prime}(t) \leq-\eta G(t)+\left(\eta\left(\frac{3}{2}+\omega^{2}\right)-\omega\right)\left\|w_{t}\right\|_{L^{2}(\Omega)}^{2} \\
+ & \left(\eta\left(\frac{3+\nu^{2}}{2}+4 c_{\Gamma}^{2}\right)-\nu\right)\left\|\zeta_{t}\right\|_{L^{2}(\Gamma)}^{2}+\int_{\Omega}\left(f\left(u^{2}\right)-f\left(u^{1}\right)\right) w \\
+ & \int_{\Omega}\left(f\left(u^{2}\right)-f\left(u^{1}\right)\right) w_{t} .
\end{aligned}
$$

Logo, pela escolha do (3.38), segue a estimativa (3.39). Usando (3.8), para alguma constante $C>0$,

$$
\begin{aligned}
\int_{\Omega}\left(f\left(u^{2}(t)\right)-f\left(u^{1}(t)\right)\right) w(t) d x & \leq \int_{\Omega} C\left(1+\left|u^{2}\right|^{2}+\left|u^{1}\right|^{2}\right)|w|^{2} \\
& \leq C^{\prime}\left(1+\left\|u^{1}(t)\right\|_{L^{4}(\Omega)}^{2}+\left\|u^{2}(t)\right\|_{L^{4}(\Omega)}^{2}\right)\|w(t)\|_{L^{4}(\Omega)}^{2} \\
& \leq C^{\prime \prime}\left(1+\left\|u^{1}(t)\right\|_{H^{1}(\Omega)}^{2}+\left\|u^{2}(t)\right\|_{H^{1}(\Omega)}^{2}\right)\|w(t)\|_{L^{4}(\Omega)}^{2} \\
& \leq C^{\prime \prime}\left(1+\left\|U(t, \tau) z_{\tau}^{1}\right\|_{\mathcal{H}}^{2}+\left\|U(t, \tau) z_{\tau}^{2}\right\|_{\mathcal{H}}^{2}\right)\|w(t)\|_{L^{4}(\Omega)}^{2} \\
& =k(\tau, t)\|w(t)\|_{L^{4}(\Omega)}^{2},
\end{aligned}
$$

Pela equivalência das energias (3.36), obtemos que

$$
G_{\eta}^{\prime}(t) \leq-\frac{2 \eta}{3} G_{\eta}(t)+k(\tau, t)\|w\|_{L^{4}(\Omega)}^{2}+\int_{\Omega}\left(f\left(u^{2}\right)-f\left(u^{1}\right)\right) w_{t} .
$$

Pelo lema de Gronwall

$$
\begin{aligned}
G_{\eta}(t) \leq & G_{\eta}(\tau) e^{-\frac{2 \eta}{3}(t-\tau)}+\sup _{s \in[\tau, t]} k(\tau, s) \int_{\tau}^{t} e^{-\frac{2 \eta}{3}(t-s)}\|w(s)\|_{L^{4}(\Omega)}^{2} d s \\
& +\int_{\tau}^{t} e^{-\frac{2 \eta}{3}(t-s)} \int_{\Omega}\left(f\left(u^{2}(s)\right)-f\left(u^{1}(s)\right)\right) w_{t}(s) d x d s .
\end{aligned}
$$

Finalmente, comparando (3.29) e (3.38) observamos que $\alpha<\eta$. Assim, escolhendo $\frac{2 \eta}{3}=\sigma_{2}$ concluímos que $\sigma_{1}<\sigma_{2}$ e usando (3.36) para substituir $G_{\eta}(t)$ por $G(t)$ chegamos em

$$
\begin{aligned}
G(t) \leq & 3 G(\tau) e^{-\sigma_{2}(t-\tau)}+2 \sup _{s \in[\tau, t]} k(\tau, s) \int_{\tau}^{t} e^{-\sigma_{2}(t-s)}\|w(s)\|_{L^{4}(\Omega)}^{2} d s \\
& +2 \int_{\tau}^{t} e^{-\sigma_{2}(t-s)} \int_{\Omega}\left(f\left(u_{2}(s)\right)-f\left(u_{1}(s)\right)\right) w_{t}(s) d x d s .
\end{aligned}
$$

Portanto, (3.31) está satisfeita, onde $C_{t, \tau}=4 \sup _{s \in[\tau, t]} k(\tau, s)$. Aqui estamos usando que $G(t)=\frac{1}{2}\left\|U(t, \tau) z_{\tau}^{1}-U(t, \tau) z_{\tau}^{2}\right\|_{\mathcal{H}}^{2}$ e que $e^{-\sigma_{2}(t-s)}<1$ para todo $s \in[t, \tau]$. 


\subsection{Existência do atrator pullback}

Motivados pela estimava (3.30), primeiro vamos definir os elementos à serem atraídos pela família absorvente e consequentemente atraídos pelo atrator. Mais precisamente, seja $\sigma_{1}>0$ dado pelos Lemas 3.4.2 e 3.4.4. Defina

$$
R_{0}=\left\{\rho: \mathbb{R} \rightarrow[0, \infty) \mid \lim _{\tau \rightarrow-\infty} e^{\sigma_{1} \tau} \rho(\tau)^{4}=0\right\}
$$

Então podemos definir um família de subconjuntos limitados de $\mathcal{H}$ da seguinte forma

$$
\widehat{D}=\left\{D(t) \mid D(t) \subset \bar{B}\left(0, \rho_{\hat{D}}(t)\right), D(t) \neq \emptyset, \forall t \in \mathbb{R}, \rho_{\widehat{D}} \in R_{0}\right\}
$$

onde $\bar{B}(0, r)=\left\{z \in \mathcal{H} \mid\|z\|_{\mathcal{H}} \leq r\right\}$. Assim, nosso universo será definido pela coleção de todas as famílias da forma (3.41), isto é,

$$
\mathcal{D}=\{\widehat{D} \mid \widehat{D} \operatorname{satisfaz}(3.41)\}
$$

Teorema 3.5.1. Suponha que as hipóteses (3.8) e (3.9) são satisfeitas e que (3.10) é satisfeita com $\sigma_{0} \leq \sigma_{1}$. Então o processo gerado pelo sistema (3.3)-(3.7) possui um $\mathcal{D}$ atrator pullback minimal. Adicionalmente, se $\sigma_{0}<\frac{\sigma_{1}}{2}$, então esse atrator pertence ao universo $\mathcal{D}$.

Demonstração. Primeiramente, para $\sigma_{0} \leq \sigma_{1}$, provaremos a existência de uma família $\mathcal{D}$ absorvente pullback $\widehat{B}_{0}$ para o processo $U(t, \tau)$ (vide Lema 3.5.2). Segundo, mostraremos a compacidade $\mathcal{D}$-assintótica do processo associado ao problema (vide Lema 3.5.4). Então a existência de um $\mathcal{D}$-atrator pullback minimal $\widehat{\mathcal{A}}$ seguirá do Teorema 2.3.9 . Agora, se $\sigma_{0}<\sigma_{1} / 2$, veremos que $\widehat{B}_{0} \in \mathcal{D}$ (vide Lema 3.5.2). Então, como $\mathcal{D}$ é fechado por inclusão, segue da parte final do Teorema 2.3.9 que $\widehat{A} \in \mathcal{D}$.

Lema 3.5.2. Se $\sigma_{0} \leq \sigma_{1}$ então o processo gerado pelo problema (3.3)-(3.4) tem uma familia $\mathcal{D}$-absorvente pullback, denotada por $\widehat{B}_{0}$. Mais precisamente, a família $\widehat{B}_{0}$ é dada por bolas fechadas $\bar{B}(0, \rho(t))$, onde

$$
\left|\rho_{0}(t)\right|^{2}=C_{2} \int_{-\infty}^{t} e^{-\sigma_{0}(t-s)}\|h(s)\|_{L^{2}(\Omega)}^{2} d s+C_{3} C_{f}+1,
$$

com $t$ percorrendo $\mathbb{R}$. Mais ainda, se $\sigma_{0}<\sigma_{1} / 2$, então $\widehat{B}_{0} \in \mathcal{D}$, ou seja, a família $\widehat{B}_{0}$ pertence ao universo $\mathcal{D}$. 
Demonstração. Seja $\widehat{D}$ uma família arbitrária $\mathcal{D}$. Então para qualquer par $\tau \leq t$ e qualquer $z_{\tau} \in D(\tau)$, obtemos, de (3.30), que

$$
\begin{aligned}
\left\|U(t, \tau) z_{\tau}\right\|_{\mathcal{H}}^{2} & \leq C_{1}\left(1+\left\|z_{\tau}\right\|_{\mathcal{H}}^{4}\right)+C_{2} \int_{\tau}^{t} e^{-\sigma_{1}(t-s)}\|h(s)\|_{L^{2}(\Omega)}^{2} d s+C_{3} C_{f} \\
& \leq C_{1}\left(1+\rho_{\widehat{D}}(\tau)^{4}\right) e^{-\sigma_{1}(t-\tau)}+C_{2} \int_{\tau}^{t} e^{-\sigma_{1}(t-s)}\|h(s)\|_{L^{2}(\Omega)}^{2} d s+C_{3} C_{f} .
\end{aligned}
$$

Como $\sigma_{0} \leq \sigma_{1}$, podemos escrever

$$
\int_{\tau}^{t} e^{-\sigma_{1}(t-s)}\|h(s)\|_{L^{2}(\Omega)}^{2} d s \leq \int_{\tau}^{t} e^{-\sigma_{0}(t-s)}\|h(s)\|_{L^{2}(\Omega)}^{2} d s,
$$

para qualquer $t \geq \tau$. Logo

$$
\begin{aligned}
\left\|U(t, \tau) z_{\tau}\right\|_{\mathcal{H}}^{2} & \leq C_{1}\left(1+\rho_{\widehat{D}}(\tau)^{4}\right) e^{-\sigma_{1}(t-\tau)}+C_{2} \int_{\tau}^{t} e^{-\sigma_{0}(t-s)}\|h(s)\|_{L^{2}(\Omega)}^{2} d s+C_{3} C_{f} \\
& \leq C_{1}\left(1+\rho_{\widehat{D}}(\tau)^{4}\right) e^{-\sigma_{1}(t-\tau)}+C_{2} \int_{-\infty}^{t} e^{-\sigma_{0}(t-s)}\|h(s)\|_{L^{2}(\Omega)}^{2} d s+C_{3} C_{f} \\
& \leq C_{1}\left(1+\rho_{\widehat{D}}(\tau)^{4}\right) e^{-\sigma_{1}(t-\tau)}+\rho_{0}(t)^{2}-1
\end{aligned}
$$

Pela condição (3.40), para cada $t$ fixado,

$$
\lim _{\tau \rightarrow-\infty} C_{1}\left(1+\rho_{\widehat{D}}(\tau)^{4}\right) e^{-\sigma_{1}(t-\tau)}=0
$$

Portanto existe $T(t, \widehat{D}) \leq t$ tal que

$$
U(t, \tau) D(\tau) \subset B_{0}(t), \quad \forall \tau<T(t, \widehat{D})
$$

Isso mostra que $\widehat{B}_{0}$ é uma família $\mathcal{D}$-absorvente pullback. Resta mostrar que $\widehat{B}_{0} \in \mathcal{D}$, para tal, de (3.43), podemos ver que

$$
\left|\rho_{0}(\tau)\right|^{2} e^{\frac{\sigma_{1}}{2} \tau}=C_{2} e^{-\left(\sigma_{0}-\frac{\sigma_{1}}{2}\right) \tau} \int_{-\infty}^{\tau} e^{\sigma_{0} s}\|h(s)\|_{L^{2}(\Omega)}^{2} d s+\left(C_{3} C_{f}+1\right) e^{\frac{\sigma_{1}}{2} \tau} .
$$

Como

$$
\int_{-\infty}^{\tau} e^{\sigma_{0} s}\|h(s)\|_{L^{2}(\Omega)}^{2} d s
$$

decresce quando $\tau \rightarrow-\infty$, temos que

$$
\left|\rho_{0}(\tau)\right|^{2} e^{\frac{\sigma_{1}}{2} \tau} \rightarrow 0
$$

quando $\tau \rightarrow-\infty$. Portanto $\rho_{0}$ satisfaz $(3.40)$ e consequentemente $\widehat{B}_{0} \in \mathcal{D}$. 
Observação 3.5.3. Uma estimativa útil é a seguinte. Para todo par $\tau<t$ e todo $z_{\tau} \in$ $D(\tau)$, segue, de (3.44), que

$$
\left\|U(t, \tau) z_{\tau}\right\|_{\mathcal{H}}^{2} \leq C_{1}\left(1+\left|\rho_{\widehat{D}}(\tau)\right|^{4}\right)+\left|\rho_{0}(t)\right|^{2}
$$

Lema 3.5.4. O processo $U(t, \tau)$ é $\mathcal{D}$-assintoticamente compacto no sentido pullback.

Demonstração. Primeiro, note que do Lema 3.5.2 sabemos que o processo $U(t, \tau)$ admite uma familia $\mathcal{D}$-absorvente pullback $\widehat{B}_{0} \in \mathcal{D}$. Agora observe que do Lema 3.4.4, para quaisquer $z_{\tau}^{1}, z_{\tau}^{2} \in B_{0}(\tau)$, tem-se

$$
\begin{aligned}
\left\|U(t, \tau) z_{\tau}^{1}-U(t, \tau) z_{\tau}^{2}\right\|_{\mathcal{H}}^{2} \leq & 3\left\|z_{\tau}^{1}-z_{\tau}^{2}\right\|_{\mathcal{H}}^{2} e^{-\sigma_{2}(t-\tau)}+C_{t, \tau} \int_{\tau}^{t}\left\|u^{1}(s)-u^{2}(s)\right\|_{L^{4}(\Omega)}^{2} d s \\
& \quad+4 \int_{\tau}^{t} \int_{\Omega}\left(f\left(u^{2}\right)-f\left(u^{1}\right)\right)\left(u_{t}^{1}-u_{t}^{2}\right) d x d s \\
\leq & 3\left(2\left|\rho_{0}(\tau)\right|\right)^{2} e^{-\sigma_{2}(t-\tau)}+C_{t, \tau} \int_{\tau}^{t}\left\|u^{1}(s)-u^{2}(s)\right\|_{L^{4}(\Omega)}^{2} d s \\
& +4 \int_{\tau}^{t} \int_{\Omega}\left(f\left(u^{2}\right)-f\left(u^{1}\right)\right)\left(u_{t}^{1}-u_{t}^{2}\right) d x d s
\end{aligned}
$$

onde $C_{t, \tau}=2 \sup _{s \in[\tau, t]} k(\tau, s)$ e $\left(u^{i}(t), u_{t}^{i}(t), \delta^{i}(t), \delta_{t}^{i}(t)\right)$ denota o fluxo $U(t, \tau) z_{\tau}^{i}$. Note que, de $(3.45)$

$$
k(\tau, t)=C^{\prime \prime}\left(1+\left\|U(t, \tau) z_{\tau}^{1}\right\|_{\mathcal{H}}^{2}+\left\|U(t, \tau) z_{\tau}^{2}\right\|_{\mathcal{H}}^{2}\right)<\infty, \quad \text { para todo } \quad t \geq \tau .
$$

Note que, de (3.43),

$$
\left|\rho_{0}(\tau)\right|^{2} e^{-\sigma_{2}(t-\tau)}=e^{\left(\sigma_{2}-\sigma_{0}\right) \tau}\left(\int_{-\infty}^{\tau} e^{\sigma_{0} s}\|h(s)\|_{L^{2}(\Omega)}^{2} d s+C_{3} C_{f}+1\right) e^{-\sigma_{2} t}, \quad \tau \leq t .
$$

Feito essas considerações, dados $t \in \mathbb{R}$ e $\epsilon>0$ existe $\tau_{\epsilon}=\tau\left(t, \widehat{B}_{0}, \epsilon\right)$ tal que

$$
12\left|\rho_{0}\left(\tau_{\epsilon}\right)\right|^{2} e^{-\sigma_{2}\left(t-\tau_{\epsilon}\right)}<\frac{\epsilon^{2}}{2} .
$$

Definimos $\Psi_{\epsilon}: B_{0}\left(\tau_{\epsilon}\right) \times B_{0}\left(\tau_{\epsilon}\right) \rightarrow \mathbb{R}$ por

$$
\begin{aligned}
\Psi_{\epsilon}\left(z_{1}, z_{2}\right)= & 2\left(C_{t, \tau_{\epsilon}} \int_{\tau_{\epsilon}}^{t}\left\|u_{1}(s)-u_{2}(s)\right\|_{L^{4}(\Omega)}^{2} d s\right)^{\frac{1}{2}} \\
& +8\left|\int_{\tau_{\epsilon}}^{t} \int_{\Omega}\left(f\left(u_{2}\right)-f\left(u_{1}\right)\right)\left(\partial_{t} u_{1}-\partial_{t} u_{2}\right) d s\right|^{\frac{1}{2}} .
\end{aligned}
$$

Assim, de (3.46), vemos que

$$
\left\|U\left(t, \tau_{\epsilon}\right) y-U\left(t, \tau_{\epsilon}\right) z\right\|_{\mathcal{H}} \leq \epsilon+\Psi_{\epsilon}(y, z),
$$


para todo $y, z \in B_{0}\left(\tau_{\epsilon}\right)$. Pelo Teorema 2.3.11, resta mostrar que $\Psi_{\epsilon}$ é uma função contrativa sobre $B_{0}\left(\tau_{\epsilon}\right)$. De fato, seja $\left\{z_{n}\right\}$ uma sequência de $B_{0}\left(\tau_{\epsilon}\right)$. Observe que, de (3.45), obtemos

$$
\left\|U\left(s, \tau_{\epsilon}\right) z_{n}\right\|_{\mathcal{H}} \leq\left(C_{1}\left(1+\left|\rho_{0}\left(\tau_{\epsilon}\right)\right|^{4}\right)+\left|\rho_{0}(t)\right|^{2}\right)^{\frac{1}{2}}<\infty
$$

para todo $s \in\left[\tau_{\epsilon}, t\right]$. Consequentemente, $\left\|u_{n}\right\|_{L^{2}\left(\tau_{\epsilon}, t ; H^{1}(\Omega)\right)}$ e $\left\|\partial_{t} u_{n}\right\|_{L^{2}\left(\tau_{\epsilon}, t ; L^{2}(\Omega)\right)}$ são limitadas. Lembrando que $U\left(s, \tau_{\epsilon}\right) z_{n}=\left(u_{n}(s) \partial_{t} u_{n}(s), \delta_{n}(s), \partial_{t} \delta_{n}(s)\right)$. Pela imersão compacta de $H^{1}(\Omega)$ em $L^{4}(\Omega)$, em $L^{2}(\Omega)$ e em $L^{5}(\Omega)$, e aplicando o Teorema 1.0 .8 conhecido com Teorema de Aubin-Lions, existe uma função $u$ e uma subsequência $\left\{u_{n_{k}}\right\}$ tal que

$$
\begin{aligned}
& u_{n_{k}} \rightarrow u \quad \text { fortemente em } \quad L^{2}\left(\tau_{\varepsilon}, t ; L^{2}(\Omega)\right), \\
& u_{n_{k}} \rightarrow u \text { fortemente em } \quad L^{2}\left(\tau_{\varepsilon}, t ; L^{5}(\Omega)\right) .
\end{aligned}
$$

Note que, como $L^{2}\left(\tau_{\epsilon}, t ; L^{2}(\Omega)\right)$ é reflexivo, logo

$$
\partial_{t} u_{n_{k}} \rightarrow \partial_{t} u \quad \text { fracamente em } \quad L^{2}\left(\tau_{\epsilon}, t ; L^{2}(\Omega)\right)
$$

Desta maneira, a subsequência correspondente $\left\{z_{n_{k}}\right\}$ é tal que a primeira parte de $\Psi_{\varepsilon}\left(z_{n_{l}}, z_{n_{k}}\right)$ converge para zero, isto é, $\left\{u_{n_{k}}\right\}$ é uma sequência de Cauchy em $L^{2}\left(\tau_{\varepsilon}, t ; L^{4}(\Omega)\right)$, ou melhor,

$$
\lim _{k \rightarrow \infty} \lim _{l \rightarrow \infty} \int_{\tau_{\epsilon}}^{t}\left\|u_{n_{k}}(s)-u_{n_{l}}(s)\right\|_{L^{4}(\Omega)}^{2} d s=0 .
$$

A segunda parte de $\Psi_{\epsilon}\left(z_{n_{k}}, z_{n_{l}}\right)$ envolve os termos com crescimento crítico e portanto apresentam um certa dificuldade. Observe que

$$
\int_{\tau_{\epsilon}}^{t} \int_{\Omega}\left(f\left(u_{n_{l}}\right)-f\left(u_{n_{k}}\right)\right)\left(\partial_{t} u_{n_{k}}-\partial_{t} u_{n_{l}}\right) d x d s=I_{1}(k, l)+I_{2}(k, l)+I_{3}(k, l),
$$

onde

$$
\begin{aligned}
I_{1}(k, l) & =-\int_{\Omega}\left[F\left(u_{n_{k}}(t)\right)+F\left(u_{n_{l}}(t)\right)\right] d x \\
I_{2}(k, l) & =\int_{\Omega}\left[F\left(u_{n_{k}}\left(\tau_{\epsilon}\right)\right)+F\left(u_{n_{l}}\left(\tau_{\epsilon}\right)\right)\right] d x \\
I_{3}(k, l) & =\int_{\tau_{\epsilon}}^{t} \int_{\Omega}\left[f\left(u_{n_{k}}(s)\right) \partial_{t} u_{n_{l}}(s)+f\left(u_{n_{l}}(s)\right) \partial_{t} u_{n_{k}}(s)\right] d x d s .
\end{aligned}
$$


Com efeito

$$
\begin{aligned}
\int_{\tau_{\epsilon}}^{t} \int_{\Omega}( & \left.f\left(u_{n_{l}}\right)-f\left(u_{n_{k}}\right)\right)\left(\partial_{t} u_{n_{k}}-\partial_{t} u_{n_{l}}\right) d x d s \\
= & \int_{\tau_{\epsilon}}^{t} \int_{\Omega}\left(f\left(u_{n_{l}}\right) \partial_{t} u_{n_{k}}-f\left(u_{n_{l}}\right) \partial_{t} u_{n_{l}}-f\left(u_{n_{k}}\right) \partial_{t} u_{n_{k}}+f\left(u_{n_{k}}\right) \partial_{t} u_{n_{l}}\right) d x d s \\
= & \int_{\tau_{\epsilon}}^{t} \int_{\Omega}\left(f\left(u_{n_{l}}\right) \partial_{t} u_{n_{k}}-\frac{d}{d t} F\left(u_{n_{l}}\right)-\frac{d}{d t} F\left(u_{n_{k}}\right)+f\left(u_{n_{k}}\right) \partial_{t} u_{n_{l}}\right) d x d s \\
= & \int_{\Omega}\left[-F\left(u_{n_{l}}(t)\right)+F\left(u_{n_{l}}\left(\tau_{\epsilon}\right)\right)\right] d x+\int_{\Omega}\left[-F\left(u_{n_{k}}(t)\right)+F\left(u_{n_{k}}\left(\tau_{\epsilon}\right)\right)\right] d x \\
& \quad+\int_{\tau_{\epsilon}}^{t} \int_{\Omega}\left(f\left(u_{n_{l}}\right) \partial_{t} u_{n_{k}}+f\left(u_{n_{k}}\right) \partial_{t} u_{n_{l}}\right) d x d s
\end{aligned}
$$

Note que, de (3.9), segue que $|F(u)| \leq C\left(1+|u|^{4}\right), u \in \mathbb{R}$ portanto $F$ satisfaz a condição de caratheodory e pelo Teorema 1.0.18 obtemos que o operador Nemytskii $N_{F}$ : $L^{5}(\Omega) \rightarrow L^{\frac{5}{4}}(\Omega)$ é contínuo. Como $L^{\frac{5}{4}} \hookrightarrow L^{1}$ e $u_{n_{k}} \rightarrow u$ fortemente em $L^{2}\left(\tau_{\epsilon}, t ; L^{5}(\Omega)\right)$, obtemos que

$$
\begin{gathered}
\left\|F\left(u_{n_{k}}\right)(x, t)\right\|_{L^{1}(\Omega)} \rightarrow\|F(u)(x, t)\|_{L^{1}(\Omega)}, \\
\left\|F\left(u_{n_{k}}\right)\left(x, \tau_{\epsilon}\right)\right\|_{L^{1}(\Omega)} \rightarrow\left\|F(u)\left(x, \tau_{\epsilon}\right)\right\|_{L^{1}(\Omega)},
\end{gathered}
$$

ou seja,

$$
\lim _{k \rightarrow \infty} \lim _{l \rightarrow \infty}\left[I_{1}(k, l)+I_{2}(k, l)\right]=-2 \int_{\Omega} F(u(t)) d x+2 \int_{\Omega} F\left(u\left(\tau_{\epsilon}\right)\right) d x
$$

Por outro lado, como $|f(u)| \leq C\left(1+|u|^{3}\right)$, obtemos que a não linearidade $f$ satisfaz a condição de caratheodory e pelo Teorema 1.0.18 obtemos que o operador Nemytskii $N_{f}: L^{6}(\Omega) \rightarrow L^{2}(\Omega)$ leva conjuntos limitados em conjuntos limitados. Agora, defina

$$
G_{l}(s)=\int_{\Omega} f\left(u_{n_{l}}(s)\right) \partial_{t} u_{n_{k}}(s) d x \quad \text { e } \quad G(s)=\int_{\Omega} f(u(s)) \partial_{t} u_{n_{k}}(s) d x .
$$

Observe que

$$
\left|G_{l}(s)\right| \leq\left(\int_{\Omega}\left|f\left(u_{n_{l}}(s)\right)\right|^{2} d x\right)^{\frac{1}{2}}\left(\int_{\Omega}\left|\partial_{t} u_{n_{k}}(s)\right|^{2} d x\right)^{\frac{1}{2}} .
$$

Logo, usando que o operador $N_{f}$ leva limitados em limitados e o fato de que $\left\|\partial_{t} u_{n}\right\|_{L^{2}\left(\tau_{\epsilon}, t ; L^{2}(\Omega)\right)}$ é limitado, obtemos que

$$
\left|G_{l}(s)\right| \leq M, \quad \text { quase sempre em } \quad\left[\tau_{\varepsilon}, t\right]
$$

Provemos que $G_{l}(s) \rightarrow G(s)$ quase sempre em $\left[\tau_{\varepsilon}, t\right]$ quando $l \rightarrow \infty$. De fato:

$$
\left|G_{l}(s)-G(s)\right| \leq \int_{\Omega}\left|f\left(u_{n_{l}}(s)\right)-f(u(s))\right|\left|\partial_{t} u_{n_{k}}(s)\right| d x \rightarrow 0,
$$


quase sempre em $\left[\tau_{\varepsilon}, t\right]$ e quando $l \rightarrow \infty$. Portanto pelo teorema da convergência dominada,

$$
\int_{\tau_{\varepsilon}}^{t} G_{l}(s) d s \rightarrow \int_{\tau_{\varepsilon}}^{t} G(s) d s \quad \text { quando } \quad l \rightarrow \infty .
$$

Assim, pelo fato de que $\partial_{t} u_{n_{k}} \rightarrow \partial_{t} u$ em $L^{2}\left(\tau_{\varepsilon}, t ; L^{2}(\Omega)\right)$, não é difícil de ver que

$$
\begin{aligned}
\lim _{k \rightarrow \infty}\left[\lim _{l \rightarrow \infty} \int_{\tau_{\varepsilon}}^{t} \int_{\Omega} f\left(u_{n_{l}}\right) \partial_{t} u_{n_{k}} d x d s\right] & =\int_{\tau_{\varepsilon}}^{t} \int_{\Omega} f(u) \partial_{t} u d x d s \\
& =\lim _{l \rightarrow \infty}\left[\lim _{k \rightarrow \infty} \int_{\tau_{\varepsilon}}^{t} \int_{\Omega} f\left(u_{n_{l}}\right) \partial_{t} u_{n_{k}} d x d s\right]
\end{aligned}
$$

Consequentemente,

$$
\begin{aligned}
\lim _{k \rightarrow \infty}\left[\lim _{l \rightarrow \infty} I_{3}(k, l)\right] & =2 \int_{\tau_{0}}^{t} \int_{\Omega} f(u(s)) \partial_{t} u(s) d x d s \\
& =2 \int_{\Omega} F(u(t)) d x-2 \int_{\Omega} F\left(u\left(\tau_{0}\right)\right) d x
\end{aligned}
$$

Então

$$
\lim _{l \rightarrow \infty} \lim _{k \rightarrow \infty} \int_{\tau_{\epsilon}}^{t} \int_{\Omega}\left(f\left(u_{n_{k}}\right)-f\left(u_{n_{l}}\right)\right)\left(\partial_{t} u_{n_{l}}-\partial_{t} u_{n_{k}}\right) d s=0
$$

Isso prova que $\Psi_{\epsilon}$ é contrativa sobre $B_{0}\left(\tau_{0}\right)$. Portando do Teorema 2.3.11 nosso processo é $\mathcal{D}$-assintoticamente compacto no sentido pullback.

\subsection{Semicontinuidade superior}

Nesta seção vamos supor que a força externa $h(x, t)$ satisfaz as seguintes condições

$$
h \in L_{\mathrm{loc}}^{2}\left(\mathbb{R}, L^{2}(\Omega)\right) \text { e } \int_{-\infty}^{t} e^{-\sigma_{0}(t-s)}\|h(s)\|_{L^{2}(\Omega)}^{2} d s<C_{h}, \quad \forall t \in \mathbb{R} .
$$

Essas condições são facilmente verificadas desde que

$$
h \in L^{2}\left(\mathbb{R} ; L^{2}(\Omega)\right) \bigcup L^{\infty}\left(\mathbb{R} ; L^{2}(\Omega)\right) .
$$

Então considere o seguinte problema $(3.3)_{\varepsilon^{-}}(3.7)$, porém trocando $h(x, t)$ por $\varepsilon h(x, t)$, isto é, a equação (3.3) é reescrita da seguinte forma

$$
u_{t t}-\Delta u+\omega u_{t}+u+f(u)=\varepsilon h(x, t), \quad x \in \Omega, \quad t \geq \tau .
$$


Como, vamos fazer $\varepsilon \rightarrow 0$, podemos assumir, sem perda de generalidade, que $\varepsilon \in$ $[0,1]$. Então, analogamente ao que foi feito na Seção 3.3, o problema $(3.3)_{\varepsilon^{-}}(3.7)$ gera um processo de evolução $U_{\varepsilon}(t, \tau): \mathcal{H} \rightarrow \mathcal{H}$, para cada $\varepsilon \in[0,1]$.

Observe que para $\varepsilon=0$, o problema $(3.3)_{\varepsilon^{-}}(3.7)$ é autônomo e gera um $C^{0}$-semigroupo $S(t)$ sobre $\mathcal{H}$. Como provado em [21], $S(t)$ admite um atrator global $A_{0}$. Mais ainda, podemos ver $S(t)$ como um processo de evolução definido por $U_{0}(t, \tau)=S(t-\tau)$. Então a família constante $\mathcal{A}_{0}=\left\{A_{0}\right\}$, para todo $t \in \mathbb{R}$, é precisamente o atrator pullback minimal de $U_{0}(t, \tau)$ com respeito ao universo $\mathcal{D}_{F}$ de famílias constantes $\widehat{D}=\{D(t)\}_{t \in \mathbb{R}}$ onde $D(t)=D$ é um subconjunto limitado do espaço de fase $\mathcal{H}$ para todo $t \in \mathbb{R}$. Veja Lema 2.3.12.

Feitas essas considerações, vamos provar que a família de absorventes, definida no Lema 3.5.2, pode ser escolhida independente de $t$ e $\varepsilon$. Com efeito, de (3.43) e (3.49),

$$
\left|\rho_{\varepsilon}(t)\right|^{2} \leq C_{2} C_{h}+C_{3} C_{f}+1, \quad \forall t \in \mathbb{R}, \quad \forall \varepsilon \in[0,1]
$$

Portanto, definindo $R=C_{2} C_{h}+C_{3} C_{f}+1$, vemos que

$$
\widehat{B}_{\varepsilon}=\left\{B_{\varepsilon}(t)\right\}_{t \in \mathbb{R}} \operatorname{com} B_{\varepsilon}(t)=\bar{B}(0, R),
$$

é uma família absorvente para o processo $U_{\varepsilon}(t, \tau)$, que absorve todos os limitados de $\mathcal{H}$. Em particular, $\widehat{B}_{\varepsilon} \in \mathcal{D}$, mas observe que $\mathcal{D}$ não é fechado por inclusão.

Sobre essas considerações,

Teorema 3.6.1. A familia de $\mathcal{D}$-atratores pullback $\mathcal{A}_{\varepsilon}$ é semicontínua superiormente quando $\varepsilon \rightarrow 0$, isto é,

$$
\lim _{\varepsilon \rightarrow 0} \operatorname{dist}\left(A_{\varepsilon}(t), A_{0}\right)=0
$$

para todo $t \in \mathbb{R}$.

Demonstração. Vamos aplicar a Proposição 2.3.14. Portanto, devemos provar que

(i) Existe $\delta>0$ e $t_{0} \in \mathbb{R}$ tal que

$$
B=\bigcup_{\epsilon \in(0, \delta)} \bigcup_{s \leq t_{0}} A_{\epsilon}(s) \text { é limitado. }
$$

(ii) Para todo $t \in \mathbb{R}$, todo $T \geq 0$, e todo conjunto limitado $D \subset \mathcal{H}$,

$$
\lim _{\varepsilon \rightarrow 0}\left(\sup _{\tau \in[t-T, t], z \in D}\left\|U_{\varepsilon}(t, \tau) z-U_{0}(t, \tau) z\right\|_{\mathcal{H}}\right)=0 .
$$


A condição $(i)$ é satisfeita, pois existe uma família absorvente uniformemente limitada $B_{0}(t)=\bar{B}(R, 0)$. De fato, pela invariância, $A_{\varepsilon}(t) \subset \bar{B}(0, R)$ para todo $t \in \mathbb{R}$ e todo $\varepsilon \in[0,1]$.

A fim de provar $(i i)$, dados $z \in D$ e $\tau \leq t$, escreva

$$
\begin{aligned}
& U_{\varepsilon}(t, \tau) z=\left(u^{1}(t), u_{t}^{1}(t), \delta^{1}(t), \delta_{t}^{1}(t)\right), \\
& U_{0}(t, \tau) z=\left(u^{2}(t), u_{t}^{2}(t), \delta^{2}(t), \delta_{t}^{2}(t)\right) .
\end{aligned}
$$

Então, podemos ver que $w=u^{1}-u^{2}$ e $\zeta=\delta^{1}-\delta^{2}$ satisfazem o seguinte sistema

$$
\begin{aligned}
& w_{t t}-\Delta w+w+\omega w_{t}=f\left(u^{2}\right)-f\left(u^{1}\right)+\varepsilon h, \quad x \in \Omega, \quad t \geq \tau, \\
& \zeta_{t t}+\zeta+\nu \zeta_{t}=-w_{t}, \quad x \in \Gamma, \quad t \geq \tau, \\
& \zeta_{t}=\partial_{n} w, \quad x \in \Gamma, \quad t \geq \tau,
\end{aligned}
$$

com condições iniciais nulas. Multiplicando a primeira equação por $w_{t}$ e a segunda equação por $\zeta_{t}$ obtemos,

$$
\begin{aligned}
\frac{d}{d t}\left[\|w\|_{H^{1}(\Omega)}^{2}\right. & \left.+\left\|w_{t}\right\|_{L^{2}(\Omega)}^{2}+\|\zeta\|_{L^{2}(\Gamma)}^{2}+\left\|\zeta_{t}\right\|_{L^{2}(\Gamma)}^{2}\right] \\
& \leq 2 \int_{\Omega}\left(f\left(u^{2}\right)-f\left(u^{1}\right)\right) w_{t} d x+2 \varepsilon \int_{\Omega} h w_{t} d x .
\end{aligned}
$$

Note que,

$$
\begin{aligned}
\int_{\Omega}\left(f\left(u^{2}\right)-f\left(u^{1}\right)\right) w_{t} d x & \leq C\left(1+\left\|u^{1}\right\|_{L^{6}(\Omega)}^{2}+\left\|u^{2}\right\|_{L^{6}(\Omega)}^{2}\right)\|w\|_{L^{6}(\Omega)}\left\|w_{t}\right\|_{L^{2}(\Omega)} \\
& \leq C(t, T, D)\left(\|w\|_{H^{1}(\Omega)}^{2}+\left\|w_{t}\right\|_{L^{2}(\Omega)}^{2}\right)
\end{aligned}
$$

onde $C(t, T, D)$ depende dos tempos $t, T$ e de $D$. Note também que

$$
\varepsilon \int_{\Omega} h w_{t} d x \leq \varepsilon^{2}\|h(t)\|_{L^{2}(\Omega)}^{2}+\frac{1}{4}\left\|w_{t}\right\|_{L^{2}(\Omega)}^{2}
$$

Portanto,

$$
\begin{aligned}
\frac{d}{d t}\left[\|w\|_{H^{1}(\Omega)}^{2}\right. & \left.+\left\|w_{t}\right\|_{L^{2}(\Omega)}^{2}+\|\zeta\|_{L^{2}(\Gamma)}^{2}+\left\|\zeta_{t}\right\|_{L^{2}(\Gamma)}^{2}\right] \\
& \leq C(t, T, D)\left(\|w\|_{H^{1}(\Omega)}^{2}+\left\|w_{t}\right\|_{L^{2}(\Omega)}^{2}\right)+\varepsilon^{2}\|h(t)\|_{L^{2}(\Omega)}^{2}
\end{aligned}
$$

e pelo Lema de Gronwall (preliminar),

$$
\left\|\left(w(t), w_{t}(t), \zeta(t), \zeta_{t}(t)\right)\right\|_{\mathcal{H}}^{2} \leq \varepsilon^{2} \int_{\tau}^{t} e^{C(t, T, D)(t-s)}\|h(s)\|_{L^{2}(\Omega)}^{2} d s,
$$


e consequentemente,

$$
\left\|U_{\varepsilon}(t, \tau) z-U_{0}(t, \tau) z\right\|_{\mathcal{H}}^{2} \leq \varepsilon^{2} \int_{t-T}^{t} e^{C(t, T, D)(t-s)}\|h(s)\|_{L^{2}(\Omega)}^{2} d s,
$$

para todo $\tau \in[t-T, t]$ e $z \in D$. Levando em conta que $h$ é localmente integrável, o limite (ii) está satisfeito. Então a semicontinuidade superior de $\mathcal{A}_{\varepsilon}$ quando $\varepsilon \rightarrow 0$ segue da Proposição 2.3.14. 


\section{Atrator uniforme para equação da}

\section{onda com fronteira acústica}

\subsection{Introdução}

Neste capítulo iremos em busca de um outro tipo de atrator, chamado atrator uniforme, para o mesmo problema abordado no capítulo anterior. Lembrando que no capítulo anterior estudamos o comportamento das soluções do seguinte problema não autônomo:

$$
\begin{aligned}
& u_{t t}-\Delta u+\omega u_{t}+u+f(u)=h(t), \quad x \in \Omega, \quad t \geq \tau, \\
& \delta_{t t}+\nu \delta_{t}+\delta=-u_{t}, \quad x \in \Gamma, \quad t \geq \tau \\
& \delta_{t}=\partial_{\mathbf{n}} u, \quad x \in \Gamma, \quad t \geq \tau \\
& u(x, \tau)=u_{\tau}^{0}(x), \quad u_{t}(x, \tau)=u_{\tau}^{1}(x), \quad x \in \Omega, \\
& \delta(x, \tau)=\delta_{\tau}^{0}(x), \quad \delta_{t}(x, \tau)=\delta_{\tau}^{1}(x), \quad x \in \Gamma .
\end{aligned}
$$

onde a força externa $h$ pertence ao espaço $L_{\text {loc }}^{2}\left(\mathbb{R} ; L^{2}(\Omega)\right)$. No capítulo anterior foi adotado um ponto de vista da dinâmica pullback. Uma outra abordagem interessante dos sistemas não autônomos é a teoria dos atratores uniformes. Neste caso devemos considerar uma família de problemas onde todas as forças externas pertencem a envoltória $\mathcal{H}(g)$, de uma força externa inicial dada $g$, gerada por todas as translações no tempo dessa força inicial e depois tomar o fecho dessas translações em um topologia apropriada. Sobre certas condições em $f$ e $g$ obtemos a existência de um atrator uniforme forte segundo a Definição 2.4.31.

Este capítulo está dividido da seguinte forma, num primeiro instante, fixamos as hipóteses e as notações preliminares. Na Seção 4.3 exibimos um resultado de existência e unicidade global do problema e por fim apresentamos algumas desigualdades importantes e demonstramos a existência do atrator uniforme forte. 


\subsection{Hipóteses e notações preliminares}

Como na Seção 3.2, suponha que o termo não linear $f \in C^{1}(\mathbb{R})$ é tal que

$$
\left|f^{\prime}(u)\right| \leq C\left(1+|u|^{2}\right)
$$

para todo $u \in \mathbb{R}$ e para alguma constante $C>0$. Admita que existem $\beta \in(0,1)$ e $m_{f}>0$ tais que

$$
F(u) \geq-\frac{\beta}{2} u^{2}-m_{f} \quad \text { e } \quad f(u) u-F(u) \geq-\frac{\beta}{2} u^{2}-m_{f}, \quad \forall u \in \mathbb{R},
$$

onde $\int_{0}^{u} f(s) d s$. Vamos supor que a força externa inicial $g \in L_{l o c}^{2}\left(\mathbb{R} ; L^{2}(\Omega)\right)$ seja de translação limitada, isto é, $g \in L_{b}^{2}\left(\mathbb{R} ; L^{2}(\Omega)\right)$. Defina a envoltória fraca da força inicial $g$ como na Definição 2.4.20,

$$
\mathcal{H}(g)=\overline{\mathcal{O}(g)} L_{\text {loc, }}^{2}\left(\mathbb{R} ; L^{2}(\Omega)\right)=\overline{\{T(s) g, s \in \mathbb{R}\}}^{L_{l o c, w}^{2}\left(\mathbb{R} ; L^{2}(\Omega)\right)} \subset L_{b}^{2}\left(\mathbb{R} ; L^{2}(\Omega)\right),
$$

onde $T: \mathcal{H}(g) \rightarrow \mathcal{H}(g)$ é o grupo de translações.

Observação 4.2.1. Como vimos na Seção 2.4, desde que $h \in L_{b}^{2}\left(\mathbb{R} ; L^{2}(\Omega)\right)$ então

$$
\sup _{t \in \mathbb{R}} \int_{t}^{t+1}\|h(s)\|_{L^{2}(\Omega)}^{2} d s=\|h\|_{L_{b}^{2}\left(\mathbb{R} ; L^{2}(\Omega)\right)}^{2}<\infty .
$$

Assim, pelo Lema 2.4.1, para qualquer $\nu>0$ e qualquer $t \in \mathbb{R}$,

$$
\int_{-\infty}^{t} e^{-\nu(t-s)}\|h(s)\|_{L^{2}(\Omega)}^{2} \leq \frac{1}{1-e^{-\nu}} \sup _{\tau \in \mathbb{R}}\left[\int_{\tau}^{\tau+1}\|h(s)\|_{L^{2}(\Omega)}^{2} d s\right]<\infty
$$

para todo $h \in \mathcal{H}(g)$.

\subsection{Existência e unicidade}

A existência e unicidade do problema (4.1)-(4.3) já foi estudada no capítulo anterior no caso em que a força externa $h \in L_{\text {loc }}^{2}\left(\mathbb{R} ; L^{2}(\Omega)\right)$. Agora estamos considerando uma família de problemas indexadas por $h \in \mathcal{H}(g)$. Como anteriormente, considere o seguinte espaço de fase

$$
\mathcal{H}=H^{1}(\Omega) \times L^{2}(\Omega) \times L^{2}(\Gamma) \times L^{2}(\Gamma)
$$

Assim, segue o seguinte teorema de boa colocação. 
Teorema 4.3.1. Suponha que $f \in C^{1}(\mathbb{R})$ satisfaz

$$
\left|f^{\prime}(u)\right| \leq C\left(1+|u|^{2}\right) \text { e } \liminf _{|u| \rightarrow \infty} \frac{f(u)}{u}>-1
$$

Suponha também que $g$ seja de translação limitada. Então, dados um tempo inicial $\tau \in \mathbb{R}$, um dado inicial $z_{\tau}=\left(u_{\tau}^{0}, u_{\tau}^{1}, \delta_{\tau}^{0}, \delta_{\tau}^{1}\right) \in \mathcal{H}$ e uma força externa $h \in \mathcal{H}(g)$, o problema (4.1)(4.3) tem uma única solução generalizada $z=\left(u, u_{t}, \delta, \delta_{t}\right)$ satisfazendo

$$
z \in C^{0}([\tau, \infty) ; \mathcal{H})
$$

Se $z_{\tau}=\left(u_{\tau}^{0}, u_{\tau}^{1}, \delta_{\tau}^{0}, \delta_{\tau}^{1}\right) \in \mathcal{H}_{1}=\left\{w \in H^{2}(\Omega) \times H^{1}(\Omega) \times H^{1 / 2}(\Gamma) \times H^{1 / 2}(\Gamma) \mid \partial_{n} w_{1}=w_{4}\right\} e$ $h \in H_{l o c}^{1}\left(\mathbb{R} ; L^{2}(\Omega)\right)$ então a solução acima é uma solução regular, ou seja, satisfaz

$$
z \in C^{0}\left([\tau, \infty) ; \mathcal{H}_{1}\right) \cap C^{1}([\tau, \infty) ; \mathcal{H})
$$

Além disso, se $z^{i}(t)=\left(u^{i}(t), u_{t}^{i}(t), \delta^{i}(t), \delta_{t}^{i}(t)\right), i=1,2$, são duas soluções generalizadas do problema (4.1)-(4.3), então para todo $T>\tau$,

$$
\left\|z^{1}(t)-z^{2}(t)\right\|_{\mathcal{H}}^{2} \leq e^{c_{0}(T-\tau)}\left\|z^{1}(\tau)-z^{2}(\tau)\right\|_{\mathcal{H}}^{2}
$$

para todo $t \in[\tau, T]$, onde $c_{0}$ é uma constante que depende dos dados iniciais.

Demonstração. Basta notar que:

$$
h \in \mathcal{H}(g) \subset L_{b}^{2}\left(\mathbb{R} ; L^{2}(\Omega)\right) \subset L_{l o c}^{2}\left(\mathbb{R} ; L^{2}(\Omega)\right),
$$

e que vale a desigualdade (4.8). Logo podemos aplicar o Teorema de existência demonstrado na capítulo anterior, Teorema 3.3.3 e assim concluir que existe uma única solução generalizada com tempo inicial $\tau$ e dado inicial $z_{\tau}$.

Observe que o problema

$$
\begin{aligned}
& u_{t t}-\Delta u+\omega u_{t}+u+f(u)=h(t), \quad x \in \Omega, \quad t \geq \tau, \quad h \in \mathcal{H}(g) \\
& \delta_{t t}+\nu \delta_{t}+\delta=-u_{t}, \quad x \in \Gamma, \quad t \geq \tau, \\
& \delta_{t}=\partial_{\mathbf{n}} u, \quad x \in \Gamma, \quad t \geq \tau, \\
& u(x, \tau)=u_{\tau}^{0}(x), \quad u_{t}(x, \tau)=u_{\tau}^{1}(x), \quad x \in \Omega, \\
& \delta(x, \tau)=\delta_{\tau}^{0}(x), \quad \delta_{t}(x, \tau)=\delta_{\tau}^{1}(x), \quad x \in \Gamma .
\end{aligned}
$$


gera uma família de processos $U_{h}(t, \tau): \mathcal{H} \rightarrow \mathcal{H}$ indexada em $h \in \mathcal{H}(g)$ e definida pelo operador solução, isto é, para cada $\tau \in \mathbb{R}$, cada dado inicial $z_{\tau}$ e cada força externa $h \in \mathcal{H}(g)$ então

$$
U_{h}(t, \tau) z_{\tau}=z\left(t ; \tau, z_{\tau}, h\right)=z(t)
$$

onde $z(t) \in \mathcal{H}, t \geq \tau$ é a solução do problema dado pelo Teorema 4.3.1, com tempo inicial $\tau$, dado inicial $z_{\tau}$ e força externa $h$.

Lema 4.3.2. A familia de processos $U_{h}(t, \tau): \mathcal{H} \rightarrow \mathcal{H}$ associada ao problema (4.1)-(4.5), é fracamente contínua em $\mathcal{H} \times \mathcal{H}(g)$ segundo a Definição 2.4.28.

Demonstração. Dados $t \geq \tau$, Suponha que

$$
z_{\tau}^{n}=\left(u_{\tau}^{0 n}, u_{\tau}^{1 n}, \delta_{\tau}^{0 n}, \delta_{\tau}^{1 n}\right) \rightarrow z_{\tau}=\left(u_{\tau}^{0}, u_{\tau}^{1}, \delta_{\tau}^{0}, \delta_{\tau}^{1}\right) \quad \text { em } \quad \mathcal{H}
$$

e

$$
h_{n} \rightarrow h \quad \text { em } \quad L_{\text {loc }}^{2}\left(\mathbb{R} ; L^{2}(\Omega)\right)
$$

Agora, denote por $z^{n}(t)$ e $z(t)$ as respectivas soluções, isto é,

$$
z^{n}(t)=U_{h_{n}}(t, \tau) z_{\tau}^{n}=\left(u^{n}(t), u_{t}^{n}(t), \delta^{n}(t), \delta_{t}^{n}(t)\right)
$$

e

$$
z(t)=U_{h}(t, \tau) z_{\tau}=\left(u(t), u_{t}(t), \delta(t), \delta_{t}(t)\right)
$$

Olhando para o seguinte sistema

$$
\begin{aligned}
& u_{t t}^{n}-\Delta u^{n}+\omega u_{t}^{n}+u^{n}+f\left(u^{n}\right)=h_{n}(t), \quad x \in \Omega, \quad t \geq \tau, \\
& \delta_{t t}^{n}+\nu \delta_{t}^{n}+\delta^{n}=-u_{t}^{n}, \quad x \in \Gamma, \quad t \geq \tau, \\
& \delta_{t}^{n}=\partial_{\mathbf{n}} u^{n}, \quad x \in \Gamma, \quad t \geq \tau, \\
& u^{n}(x, \tau)=u_{\tau}^{0 n}(x), \quad u_{t}(x, \tau)=u_{\tau}^{1 n}(x), \quad x \in \Omega, \\
& \delta^{n}(x, \tau)=\delta_{\tau}^{0 n}(x), \quad \delta_{t}^{n}(x, \tau)=\delta_{\tau}^{1 n}(x), \quad x \in \Gamma,
\end{aligned}
$$

podemos concluir que

$$
\begin{aligned}
\left\|u^{n}(t)\right\|_{H^{1}(\Omega)}^{2}+\left\|u_{t}^{n}(t)\right\|_{L^{2}(\Omega)}^{2}+\left\|\delta^{n}(t)\right\|_{L^{2}(\Gamma)}^{2}+\left\|\delta_{t}^{n}(t)\right\|_{L^{2}(\Gamma)} \\
\leq C\left(\left\|z_{\tau}^{n}\right\|_{\mathcal{H}}^{2}+\int_{\tau}^{t}\left\|h_{n}(s)\right\|_{L^{2}(\Omega)}^{2} d s+1\right) .
\end{aligned}
$$


Como $z_{\tau}^{n} \rightarrow z_{\tau}$ em $\mathcal{H}$ e $h_{n} \rightarrow h$ em $L_{\text {loc }}^{2}\left(\mathbb{R} ; L^{2}(\Omega)\right)$, temos que

$$
\left\|u^{n}(t)\right\|_{H^{1}(\Omega)}^{2}+\left\|u_{t}^{n}(t)\right\|_{L^{2}(\Omega)}^{2}+\left\|\delta^{n}(t)\right\|_{L^{2}(\Gamma)}^{2}+\left\|\delta_{t}^{n}(t)\right\|_{L^{2}(\Gamma)} \leq C .
$$

Logo concluímos que

$$
z^{n}(t)=\left(u^{n}(t), u_{t}^{n}(t), \delta^{n}(t), \delta_{t}^{n}(t)\right) \rightarrow \bar{z}(t)=\left(\bar{u}(t), \bar{u}_{t}(t), \bar{\delta}(t), \bar{\delta}_{t}(t)\right) \quad \text { em } \quad \mathcal{H} .
$$

Similarmente a etapa de passagem de limite conhecida no método de Galerkin, podemos passar o limite no problema e assim concluímos que $\bar{z}$ é também uma solução com dado inicial $z_{\tau}$. Portanto pela unicidade $\bar{z}(t)=z(t)=U_{h}(t, \tau) z_{\tau}$, provando o desejado.

\subsection{Desigualdades notáveis}

Sabendo que o processo $U_{h}(t, \tau), h \in \mathcal{H}(g)$,associado ao problema (4.1)-(4.5) é fracamente contínuo em $\mathcal{H} \times \mathcal{H}(g)$, primeiramente vamos em busca do atrator uniforme fraco, para tal provaremos um lema que é fundamental para exibir um conjunto absorvente uniforme, com respeito à $h \in \mathcal{H}(g)$, segundo a Definição 2.4.27. Porém, queremos exibir um atrator uniforme forte e para isso precisamos provar a compacidade assintótica uniforme da família de processos, assim, tendo em vista esses objetivos vamos demonstrar um lema crucial para verificar tal propriedade da família de processos.

As desigualdades obtidas nessa seção são análogas as desigualdades obtidas na Seção 3.4. Lembrando que a energia ao longo de uma solução do problema (4.1)-(4.5) é dada por

$$
\mathcal{E}(t)=\frac{1}{2}\|u(t)\|_{H^{1}(\Omega)}^{2}+\frac{1}{2}\left\|u_{t}(t)\right\|_{L^{2}(\Omega)}^{2}+\frac{1}{2}\|\delta(t)\|_{L^{2}(\Gamma)}^{2}+\frac{1}{2}\left\|\delta_{t}(t)\right\|_{L^{2}(\Gamma)}^{2}+\int_{\Omega} F(u(t)) d x,
$$

e

$$
\mathcal{E}^{\prime}(t)=-\omega\left\|u_{t}\right\|_{L^{2}(\Omega)}^{2}-\nu\left\|\delta_{t}\right\|_{L^{2}(\Gamma)}^{2}+\int_{\Omega} h u_{t} d x,
$$

para cada $h \in \mathcal{H}(g)$. Como antes, existem constantes $\beta_{0}, C_{f}, C_{F}$ satisfazendo (3.23)-(3.24), isto é,

$$
\mathcal{E}(t) \geq \beta_{0}\left\|\left(u, u_{t}, \delta, \delta_{t}\right)\right\|_{\mathcal{H}}^{2}-C_{f}, \quad t \geq \tau,
$$

$\mathrm{e}$

$$
\mathcal{E}(t) \leq C_{F}\left(1+\left\|\left(u, u_{t}, \delta, \delta_{t}\right)\right\|_{\mathcal{H}}^{4}\right), \quad t \geq \tau
$$


O lema a seguir irá auxiliar na construção do conjunto absorvente uniforme para a família de processos $U_{h}(t, \tau)$.

Lema 4.4.1. Existem constantes $C_{1}, C_{2}, C_{3}, \sigma_{1}>0$ tais que, para qualquer $z_{\tau} \in \mathcal{H}$ e para qualquer $h \in \mathcal{H}(g)$,

$$
\left\|U_{h}(t, \tau) z_{\tau}\right\|_{\mathcal{H}}^{2} \leq C_{1}\left(1+\left\|z_{\tau}\right\|_{\mathcal{H}}^{4}\right) e^{-\sigma_{1}(t-\tau)}+\left(\frac{C_{2}}{1-e^{-\sigma_{1}}}\right)\|g\|_{L_{b}^{2}\left(\mathbb{R} ; L^{2}(\Omega)\right)}^{2}+C_{3} C_{f}
$$

para quaisquer $t \geq \tau$.

Demonstração. A prova deste lema é similar à prova do Lema 3.4.2, portanto iremos demonstra-la de forma sucinta. Começamos definindo a energia perturbada

$$
\mathcal{E}_{\alpha}(t)=\mathcal{E}(t)+\alpha \Phi(t), \quad \alpha>0
$$

onde,

$$
\Phi(t)=\int_{\Omega} u(t) u_{t}(t) d x+\int_{\Gamma} \delta(t) \delta_{t}(t) d \gamma+\int_{\Gamma} u(t) \delta(t) d \gamma
$$

Para concluir, dividimos a demonstração em três etapas.

Etapa 1. Existe $\alpha_{0}>0$ tal que

$$
\frac{1}{2} \mathcal{E}(t)-\frac{1}{2} C_{f} \leq \mathcal{E}_{\alpha}(t) \leq \frac{3}{2} \mathcal{E}(t)+\frac{1}{2} C_{f}, \quad \forall \alpha \in\left[0, \alpha_{0}\right]
$$

Etapa 2. Podemos estimar $\Phi^{\prime}(t)$ como

$$
\begin{aligned}
\Phi^{\prime}(t) \leq & -\mathcal{E}(t)+\left(\frac{3}{2}+\frac{2 \omega^{2}}{1-\beta}\right)\left\|u_{t}(t)\right\|_{L^{2}(\Omega)}^{2}+\left(\frac{3+\nu^{2}}{2}+\frac{4 c_{\Gamma}^{2}}{1-\beta}\right)\left\|\delta_{t}(t)\right\|_{L^{2}(\Gamma)}^{2} \\
& +\frac{2}{1-\beta}\|h(t)\|_{L^{2}(\Omega)}^{2}+C_{f}
\end{aligned}
$$

Etapa 3. Conclusão. Levando em conta que

$$
\mathcal{E}^{\prime}(t) \leq-\frac{\omega}{2}\left\|u_{t}\right\|_{L^{2}(\Omega)}^{2}-\nu\left\|\delta_{t}\right\|_{L^{2}(\Gamma)}^{2}+\frac{1}{2 \omega}\|h(t)\|_{L^{2}(\Omega)}^{2},
$$

escolhemos

$$
\alpha=\min \left\{\alpha_{0}, \frac{\omega}{2}\left(\frac{3}{2}+\frac{2 \omega^{2}}{1-\beta}\right)^{-1}, \nu\left(\frac{3+\nu^{2}}{2}+\frac{4 c_{\Gamma}^{2}}{1-\beta}\right)^{-1}\right\} .
$$

Então, como $\alpha \leq \alpha_{0} \leq(1-\beta) / 4$. Tem-se

$$
\mathcal{E}_{\alpha}^{\prime}(t) \leq-\alpha \mathcal{E}(t)+\left(\frac{1}{2}+\frac{1}{2 \omega}\right)\|h(t)\|_{L^{2}(\Omega)}^{2}+\alpha C_{f} .
$$


Defina $C_{\omega}=\frac{1}{2}+\frac{1}{2 \omega}$. Assim, usando (4.11) podemos ver que

$$
\mathcal{E}_{\alpha}^{\prime}(t) \leq-\frac{2 \alpha}{3} \mathcal{E}_{\alpha}(t)+C_{\omega}\|h(t)\|_{L^{2}(\Omega)}^{2}+\frac{4 \alpha}{3} C_{f},
$$

e pela desigualdade de Gronwall, Lema 1.0.7,

$$
\mathcal{E}_{\alpha}(t) \leq \mathcal{E}_{\alpha}(\tau) e^{-\frac{2 \alpha}{3}(t-\tau)}+C_{\omega} \int_{\tau}^{t} e^{-\frac{2 \alpha}{3}(t-s)}\|h(s)\|_{L^{2}(\Omega)}^{2} d s+2 C_{f} .
$$

Usando novamente (4.11), e definindo $\sigma_{1}=2 \alpha / 3$, obtemos:

$$
\mathcal{E}(t) \leq 6 \mathcal{E}(\tau) e^{-\sigma_{1}(t-\tau)}+2 C_{\omega} \int_{\tau}^{t} e^{-\sigma_{1}(t-s)}\|h(s)\|_{L^{2}(\Omega)}^{2} d s+6 C_{f}, \quad t \geq \tau .
$$

Combinando (4.14) com (3.23)-(3.24), existe $C_{1}, C_{2}, C_{3}>0$ tal que, para qualquer $z \in \mathcal{H}$

$$
\|U(t, \tau) z\|_{\mathcal{H}}^{2} \leq C_{1}\left(1+\|z\|_{\mathcal{H}}^{4}\right) e^{-\sigma_{1}(t-\tau)}+C_{2} \int_{\tau}^{t} e^{-\sigma_{1}(t-s)}\|h(s)\|_{L^{2}(\Omega)}^{2} d s+C_{3} C_{f},
$$

para todo par $\tau \leq t$. Logo, de (4.8),

$$
\|U(t, \tau) z\|_{\mathcal{H}}^{2} \leq C_{1}\left(1+\|z\|_{\mathcal{H}}^{4}\right) e^{-\sigma_{1}(t-\tau)}+\left(\frac{C_{2}}{1-e^{-\sigma_{1}}}\right)\|h\|_{L_{b}^{2}\left(\mathbb{R} ; L^{2}(\Omega)\right)}^{2} d s+C_{3} C_{f} .
$$

Finalmente, usando o item 1) da Proposição 2.4.21, obtemos

$$
\|U(t, \tau) z\|_{\mathcal{H}}^{2} \leq C_{1}\left(1+\|z\|_{\mathcal{H}}^{4}\right) e^{-\sigma_{1}(t-\tau)}+\left(\frac{C_{2}}{1-e^{-\sigma_{1}}}\right)\|g\|_{L_{b}^{2}\left(\mathbb{R} ; L^{2}(\Omega)\right)}^{2} d s+C_{3} C_{f},
$$

para todo $h \in \mathcal{H}(g)$.

O próximo lema será fundamental na prova da compacidade assintótica uniforme da família de processos.

Lema 4.4.2. Dados quaisquer $z_{\tau}^{1}, z_{\tau}^{2} \in \mathcal{H}$ e quaisquer $h_{1}, h_{2} \in \mathcal{H}(g)$. Temos que

$$
\begin{aligned}
\left\|U_{h_{1}}(t, \tau) z_{\tau}^{1}-U_{h_{2}}(t, \tau) z_{\tau}^{2}\right\|_{\mathcal{H}}^{2} \leq & 3\left\|z_{\tau}^{1}-z_{\tau}^{2}\right\|_{\mathcal{H}}^{2} e^{-\sigma_{1}(t-\tau)}+C_{t, \tau} \int_{\tau}^{t}\left\|u^{1}(s)-u^{2}(s)\right\|_{L^{4}(\Omega)}^{2} d s \\
& +4 \int_{\tau}^{t} \int_{\Omega}\left(f\left(u^{2}(s)\right)-f\left(u^{1}(s)\right)\right)\left(u_{t}^{1}(s)-u_{t}^{2}(s)\right) d x d s \\
& +4 \int_{\tau}^{t} e^{-\sigma_{1}(t-s)} \int_{\Omega}\left(h_{1}(s)-h_{2}(s)\right)\left(u^{1}(s)-u^{2}(s)\right) d x d s \\
& +4 \int_{\tau}^{t} e^{-\sigma_{1}(t-s)} \int_{\Omega}\left(h_{1}(s)-h_{2}(s)\right)\left(u_{t}^{1}(s)-u_{t}^{2}(s)\right) d x d s
\end{aligned}
$$

onde $U_{h_{i}}(t, \tau) z_{\tau}^{i}=\left(u^{i}(t), \partial_{t} u^{i}(t), \delta^{i}(t), \partial_{t} \delta^{i}(t)\right)$ e $C_{t, \tau}>0$ é uma constante. 
Demonstração. A prova é dividida em três etapas.

Etapa 1. Denote $w=u^{1}-u^{2}$ e $\zeta=\delta^{1}-\delta^{2}$. Então $w, \zeta$ satisfazem

$$
\begin{aligned}
& w_{t t}-\Delta w+w+\omega w_{t}=f\left(u^{2}\right)-f\left(u^{1}\right)+h_{1}-h_{2}, \quad x \in \Omega, \quad t \geq \tau, \\
& \zeta_{t t}+\nu \zeta_{t}+\zeta=-w_{t}, \quad x \in \Gamma, \quad t \geq \tau, \\
& \zeta_{t}=\partial_{\mathbf{n}} w, \quad x \in \Gamma, \quad t \geq \tau,
\end{aligned}
$$

com condições iniciais

$$
\begin{aligned}
& \xi(x, \tau)=u^{1}(x, \tau)-u^{2}(x, \tau),\left.\quad \partial_{t} \xi(x, t)\right|_{t=\tau}=u_{t}^{1}(x, \tau)-u_{t}^{2}(x, \tau), \quad x \in \Omega, \\
& \zeta(x, \tau)=\delta^{1}(x, \tau)-\delta^{2}(x, \tau),\left.\quad \partial_{t} \zeta(x, t)\right|_{t=\tau}=\delta_{t}^{1}(x, \tau)-\delta_{t}^{2}(x, \tau), \quad x \in \Gamma .
\end{aligned}
$$

Defina o seguinte funcional

$$
G(t)=\frac{1}{2}\left\|\left(w(t), w_{t}(t), \zeta(t), \zeta_{t}(t)\right)\right\|_{\mathcal{H}}^{2}, \quad t \in \mathbb{R}
$$

Então, deduzimos que

$$
G^{\prime}(t)=-\omega\left\|w_{t}\right\|_{L^{2}(\Omega)}^{2}-\nu\left\|\zeta_{t}\right\|_{L^{2}(\Gamma)}^{2}+\int_{\Omega}\left(f\left(u^{2}\right)-f\left(u^{1}\right)\right) w_{t} d x+\int_{\Omega}\left(h_{1}-h_{2}\right) w_{t} d x
$$

Etapa 2. Agora vamos definir a energia perturbada por

$$
G_{\eta}(t)=G(t)+\eta \Psi(t)
$$

onde

$$
\Psi(t)=\int_{\Omega} w(t) w_{t}(t) d x+\int_{\Gamma} \zeta(t) \zeta_{t}(t) d \gamma+\int_{\Gamma} w(t) \zeta(t) d \gamma .
$$

Note que, como $|\Psi(t)| \leq\left(1+c_{\Gamma}^{2}\right) G(t)$, segue que

$$
\frac{1}{2} G(t) \leq G_{\eta}(t) \leq \frac{3}{2} G(t), \quad \forall t \in \mathbb{R}, \quad \forall \eta \leq \eta_{0}=\frac{1}{2\left(1+c_{\Gamma}^{2}\right)} .
$$

Procedendo como na prova do Lema 3.4.2 (Lema anterior), podemos ver que

$$
\begin{aligned}
\Psi^{\prime}(t) \leq & -G(t)+\left(\frac{3}{2}+\omega^{2}\right)\left\|w_{t}\right\|_{L^{2}(\Omega)}^{2}+\left(\frac{3+\nu^{2}}{2}+4 c_{\Gamma}^{2}\right)\left\|\zeta_{t}\right\|_{L^{2}(\Gamma)}^{2} \\
& +\int_{\Omega}\left(f\left(u^{2}\right)-f\left(u^{1}\right)\right) w d x+\int_{\Omega}\left(h_{1}-h_{2}\right) w d x
\end{aligned}
$$

Em vista de $G^{\prime}(t)$, escolhendo

$$
\eta=\min \left\{\eta_{0}, \omega\left(\frac{3}{2}+\omega^{2}\right)^{-1}, \nu\left(\frac{3+\nu^{2}}{2}+4 c_{\Gamma}^{2}\right)^{-1}\right\}
$$


obtemos

$$
\begin{aligned}
G_{\eta}^{\prime}(t) \leq- & \eta G(t)+\int_{\Omega}\left(f\left(u^{2}(t)\right)-f\left(u^{1}(t)\right)\right) w(t) d x+\int_{\Omega}\left(f\left(u^{2}(t)\right)-f\left(u^{1}(t)\right)\right) w_{t}(t) d x \\
+ & \int_{\Omega}\left(h_{1}(t)-h_{2}(t)\right) w(t) d x+\int_{\Omega}\left(h_{1}(t)-h_{2}(t)\right) w_{t}(t) d x
\end{aligned}
$$

Usando (3.8), para alguma constante $C>0$,

$$
\begin{aligned}
\int_{\Omega}\left(f\left(u^{2}(t)\right)-f\left(u^{1}(t)\right)\right) w(t) d x & \leq C\left(1+\left\|u^{1}(t)\right\|_{L^{4}(\Omega)}^{2}+\left\|u^{2}(t)\right\|_{L^{4}(\Omega)}^{2}\right)\|w(t)\|_{L^{4}(\Omega)}^{2} \\
& \leq C\left(1+\left\|U_{h_{1}}(t, \tau) z_{\tau}^{1}\right\|_{\mathcal{H}}^{2}+\left\|U_{h_{2}}(t, \tau) z_{\tau}^{2}\right\|_{\mathcal{H}}^{2}\right)\|w(t)\|_{L^{4}(\Omega)}^{2} \\
& =k(\tau, t)\|w(t)\|_{L^{4}(\Omega)}^{2},
\end{aligned}
$$

onde,

$$
k(\tau, t)=C\left(1+\left\|U_{h_{1}}(t, \tau) z_{\tau}^{1}\right\|_{\mathcal{H}}^{2}+\left\|U_{h_{2}}(t, \tau) z_{\tau}^{2}\right\|_{\mathcal{H}}^{2}\right), \quad \forall \tau \leq t
$$

Então usando a equivalência da energia (4.16) concluímos que

$$
\begin{aligned}
G_{\eta}(t) \leq & G_{\eta}(\tau) e^{-\frac{2 \eta}{3}(t-\tau)}+\sup _{s \in[\tau, t]} k(\tau, s) \int_{\tau}^{t} e^{-\frac{2 \eta}{3}(t-s)}\|w(s)\|_{L^{4}(\Omega)}^{2} d s \\
& +\int_{\tau}^{t} e^{-\frac{2 \eta}{3}(t-s)} \int_{\Omega}\left(f\left(u^{2}(s)\right)-f\left(u^{1}(s)\right)\right) w_{t}(s) d x d s \\
& +\int_{\tau}^{t} e^{-\frac{2 \eta}{3}(t-s)} \int_{\Omega}\left(h_{1}(s)-h_{2}(s)\right) w(s) d x d s \\
& +\int_{\tau}^{t} e^{-\frac{2 \eta}{3}(t-s)} \int_{\Omega}\left(h_{1}(s)-h_{2}(s)\right) w_{t}(s) d x d s .
\end{aligned}
$$

Finalmente, comparando (4.13) e (4.17) observamos que $\alpha \leq \eta$. Então podemos escolher $\eta$ tal que $\frac{2 \eta}{3}=\sigma_{1}$. Usando (4.16) para substituir $G_{\eta}(t)$ por $G(t)$ podemos obter

$$
\begin{aligned}
G(t) \leq & 3 G(\tau) e^{-\sigma_{1}(t-\tau)}+2 \sup _{s \in[\tau, t]} k(\tau, s) \int_{\tau}^{t} e^{-\sigma_{1}(t-s)}\|w(s)\|_{L^{4}(\Omega)}^{2} d s \\
& +2 \int_{\tau}^{t} e^{-\sigma_{1}(t-s)} \int_{\Omega}\left(f\left(u_{2}(s)\right)-f\left(u_{1}(s)\right)\right) w_{t}(s) d x d s . \\
& +2 \int_{\tau}^{t} e^{-\sigma_{1}(t-s)} \int_{\Omega}\left(h_{1}(s)-h_{2}(s)\right) w(s) d x d s \\
& +2 \int_{\tau}^{t} e^{-\sigma_{1}(t-s)} \int_{\Omega}\left(h_{1}(s)-h_{2}(s)\right) w_{t}(s) d x d s .
\end{aligned}
$$

Isto conclui a prova. 


\subsection{Existência do atrator uniforme}

Nesta seção vamos em busca do atrator uniforme. Conforme a Seção 2.4, vamos exibir um atrator uniforme fraco para a família de processos $U_{h}(t, \tau), h \in \mathcal{H}(g)$ e em seguida mostraremos que esse atrator uniforme é forte no sentido da Definição 2.4.31, desde que a força externa $g$ seja de translação limitada e regular no tempo.

Teorema 4.5.1. Suponha que as hipóteses (4.6)-(4.7) são satisfeitas e, também, que $g \in L_{\text {loc }}^{2}\left(\mathbb{R} ; L^{2}(\Omega)\right)$ seja de translação limitada. Considere $U_{h}(t, \tau): \mathcal{H} \rightarrow \mathcal{H}, h \in \mathcal{H}(g)$ a família de processos associados ao problema (4.1)-(4.5). Então o semigrupo extendido associado $\mathbb{S}(t): \mathcal{H} \times \mathcal{H}(g) \rightarrow \mathcal{H} \times \mathcal{H}(g)$ admite um atrator global fraco $\mathbb{A}$. Mais ainda o atrator uniforme associado $\pi_{1} \mathbb{A}=\mathcal{A}$ é definido por todas as soluções limitadas do problema (4.1)-(4.5) com $h \in \mathcal{H}(g)$,

$$
\mathcal{A}=\left.\bigcup_{h \in \mathcal{H}(g)} \mathcal{K}_{h}\right|_{t=0}
$$

Demonstração. Segundo o Teorema 2.4.29 precisamos mostrar que a família de processos $U_{h}(t, \tau): \mathcal{H} \rightarrow \mathcal{H}, h \in \mathcal{H}(g)$ é fracamente contínua em $\mathcal{H} \times \mathcal{H}(g)$ e admite um conjunto limitado absorvente uniforme. Pelo Lema 4.3.2, segue que a família é fracamente contínua. Resta mostrar que existe um conjunto limitado uniformemente absorvente. De fato, tendo em vista a estimativa do Lema 4.4.1, defina

$$
R_{0}^{2}=\left(\frac{C_{2}}{1-e^{-\sigma_{1}}}\right)\|g\|_{L_{b}^{2}\left(\mathbb{R} ; L^{2}(\Omega)\right)}^{2}+C_{3} C_{f}+1 .
$$

Em seguida, defina a seguinte bola fechada centrada na origem com raio $R_{0}$,

$$
B_{0}=\left\{z \in \mathcal{H} ;\|z\|_{\mathcal{H}} \leq R_{0}\right\}
$$

Mostremos que $B_{0}$ um conjunto uniformemente absorvente. Seja $B$ um subconjunto limitado de $\mathcal{H}$, de (4.10), dado qualquer $z \in B$, qualquer $\tau \in \mathbb{R}$ e qualquer $h \in \mathcal{H}(g)$,

$$
\begin{aligned}
\left\|U_{h}(t, \tau) z\right\|_{\mathcal{H}}^{2} & \leq C_{1}\left(1+\|z\|_{\mathcal{H}}^{4}\right) e^{\sigma_{1}(t-\tau)}+\frac{C_{2}}{1-e^{-\sigma_{1}}}\|g\|_{L_{b}^{2}\left(\mathbb{R} ; L^{2}(\Omega)\right)}^{2}+C_{3} C_{f} \\
& \leq C_{1}\left(1+\|z\|_{\mathcal{H}}^{4}\right) e^{-\sigma_{1}(t-\tau)}+R_{0}^{2}-1 .
\end{aligned}
$$

Como $z \in B$, tem-se que $\|z\|_{\mathcal{H}}$ é limitada e, portanto, existe $T_{0}=T_{0}(B, \tau)>\tau$ tal que

$$
C_{1}\left(1+\|z\|_{\mathcal{H}}^{4}\right) e^{-\sigma_{1}(t-\tau)} \leq 1, \quad \text { para todo } t \geq T_{0}
$$


Consequentemente,

$$
\left\|U_{h}(t, \tau) z\right\|_{\mathcal{H}}^{2} \leq R_{0}^{2}
$$

Portanto, para todo $\tau \in \mathbb{R}$, todo conjunto limitado $B \subset \mathcal{H}$, existe $T_{0}(B, \tau)>\tau$ de modo que

$$
\bigcup_{h \in \mathcal{H}(g)} U_{h}(t, \tau) B \subset B_{0}
$$

para todo $t \geq T_{0}$.

Logo, estamos na hipóteses do Teorema 2.4.29, o que conclui a existência do atrator uniforme fraco para a família de processos $U_{h}(t, \tau), h \in \mathcal{H}(g)$.

Observação 4.5.2. Note que na prova do teorema anterior basta que a força externa $g$ seja de translação limitada, isto é, não precisamos que g seja regular no tempo.

Por fim chegamos ao resultado principal desse capítulo. Iremos demonstrar que o atrator uniforme fraco para a família de processos $U_{h}(t, \tau), h \in \mathcal{H}(g)$, associada ao problema (4.1)-(4.5), obtido no teorema anterior é na verdade um atrator uniforme forte, desde que $g$ seja regular no tempo.

Teorema 4.5.3. Nas hipóteses do Teorema 4.5.1 e, mais ainda, supondo que g é regular no tempo. Então o atrator uniforme fraco exibido no Teorema 4.5.1 é um atrator uniforme forte para a familia de processos $U_{h}(t, \tau), h \in \mathcal{H}(g)$ associada ao problema (4.1)-(4.5).

Demonstração. Segundo o Teorema 2.4.33, para concluírmos que o atrator uniforme fraco é um atrator uniforme forte basta apenas verificar que a família de processos $U_{h}(t, \tau)$, $h \in \mathcal{H}(g)$ é assintoticamente uniformemente compacta. Para tal, usaremos o critério introduzido no Teorema 2.4.36 e seguiremos de forma análoga ao Lema 3.5.4.

Na prova do Teorema 4.5.1 vimos que existe um conjunto absorvente uniforme e limitado $B_{0}$. Note que, pelo Lema 4.4.2,

$$
\begin{aligned}
\| U_{h_{1}}(T, 0) z^{1}-U_{h_{2}}(T, 0) & z^{2}\left\|_{\mathcal{H}}^{2} \leq 3\right\| z^{1}-z^{2}\left\|_{\mathcal{H}}^{2} e^{-\sigma_{1} T}+C_{T, 0} \int_{0}^{T}\right\| u^{1}(s)-u^{2}(s) \|_{L^{4}(\Omega)}^{2} d s \\
& +4 \int_{0}^{T} \int_{\Omega}\left(f\left(u^{2}(s)\right)-f\left(u^{1}(s)\right)\right)\left(u_{t}^{1}(s)-u_{t}^{2}(s)\right) d x d s \\
& +4 \int_{0}^{T} e^{-\sigma_{1}(T-s)} \int_{\Omega}\left(h_{1}(s)-h_{2}(s)\right)\left(u^{1}(s)-u^{2}(s)\right) d x d s \\
& +4 \int_{0}^{T} e^{-\sigma_{1}(T-s)} \int_{\Omega}\left(h_{1}(s)-h_{2}(s)\right)\left(u_{t}^{1}(s)-u_{t}^{2}(s)\right) d x d s
\end{aligned}
$$


para quaisquer $z^{1}, z^{2} \in B_{0}$, quaisquer $h_{1}, h_{2} \in \mathcal{H}(g)$, onde

$$
U_{h_{i}}(t, 0) z^{i}=\left(u^{i}(t), \partial_{t} u^{i}(t), \delta^{i}(t), \partial_{t} \delta^{i}(t)\right)
$$

Observe que, dado $\varepsilon>0$ existe $T=T\left(B_{0}, \varepsilon\right)>0$ tal que

$$
3\left\|z^{1}-z^{2}\right\|_{\mathcal{H}}^{2} e^{-\sigma_{1} T}<\frac{\varepsilon^{2}}{2} .
$$

Logo,

$$
\begin{aligned}
\left\|U_{h_{1}}(T, 0) z^{1}-U_{h_{2}}(T, 0) z^{2}\right\|_{\mathcal{H}} \leq\left\{\frac{\varepsilon^{2}}{2}+C_{T, 0} \int_{0}^{T}\left\|u^{1}(s)-u^{2}(s)\right\|_{L^{4}(\Omega)}^{2} d s\right. & \\
+ & 4 \int_{0}^{T} \int_{\Omega}\left(f\left(u^{2}(s)\right)-f\left(u^{1}(s)\right)\right)\left(u_{t}^{1}(s)-u_{t}^{2}(s)\right) d x d s \\
+ & 4 \int_{0}^{T} e^{-\sigma_{1}(T-s)} \int_{\Omega}\left(h_{1}(s)-h_{2}(s)\right)\left(u^{1}(s)-u^{2}(s)\right) d x d s \\
+ & \left.4 \int_{0}^{T} e^{\left.-\sigma_{1} T-s\right)} \int_{\Omega}\left(h_{1}(s)-h_{2}(s)\right)\left(u_{t}^{1}(s)-u_{t}^{2}(s)\right) d x d s\right\}^{\frac{1}{2}} \\
& \leq \varepsilon+2\left\{C_{T, 0} \int_{0}^{T}\left\|u^{1}(s)-u^{2}(s)\right\|_{L^{4}(\Omega)}^{2} d s\right\}^{\frac{1}{2}} \\
+ & \left\{\int_{0}^{T} \int_{\Omega}\left(f\left(u^{2}(s)\right)-f\left(u^{1}(s)\right)\right)\left(u_{t}^{1}(s)-u_{t}^{2}(s)\right) d x d s\right\}^{\frac{1}{2}} \\
+ & \left\{\int_{0}^{T} e^{-\sigma_{1}(T-s)} \int_{\Omega}\left(h_{1}(s)-h_{2}(s)\right)\left(u^{1}(s)-u^{2}(s)\right) d x d s\right\}^{\frac{1}{2}} \\
+ & 8\left\{\int_{0}^{T} e^{-\sigma_{1}(T-s)} \int_{\Omega}\left(h_{1}(s)-h_{2}(s)\right)\left(u_{t}^{1}(s)-u_{t}^{2}(s)\right) d x d s\right\}^{\frac{1}{2}} .
\end{aligned}
$$

Assim, dado $\varepsilon>0$ existe $T\left(B_{0}, \varepsilon\right)>0$ tal que

$$
\left\|U_{h_{1}}(T, 0) z^{1}-U_{h_{2}}(T, 0) z^{2}\right\|_{\mathcal{H}} \leq \varepsilon+\Phi_{T}\left(z^{1}, z^{2} ; h_{1}, h_{2}\right)
$$

para quaisquer $z^{1}, z^{2} \in B_{0}$ e quaisquer $h_{1}, h_{2} \in \mathcal{H}(g)$, onde

$$
\begin{aligned}
\Phi_{T}\left(z^{1}, z^{2} ; h_{1}, h_{2}\right)= & 2\left\{C_{T, 0} \int_{0}^{T}\left\|u^{1}(s)-u^{2}(s)\right\|_{L^{4}(\Omega)}^{2} d s\right\}^{\frac{1}{2}} \\
& +8\left\{\int_{0}^{T} \int_{\Omega}\left(f\left(u^{2}(s)\right)-f\left(u^{1}(s)\right)\right)\left(u_{t}^{1}(s)-u_{t}^{2}(s)\right) d x d s\right\}^{\frac{1}{2}} \\
& +8\left\{\int_{0}^{T} e^{-\sigma_{1}(T-s)} \int_{\Omega}\left(h_{1}(s)-h_{2}(s)\right)\left(u^{1}(s)-u^{2}(s)\right) d x d s\right\}^{\frac{1}{2}} \\
& +8\left\{\int_{0}^{T} e^{-\sigma_{1}(T-s)} \int_{\Omega}\left(h_{1}(s)-h_{2}(s)\right)\left(u_{t}^{1}(s)-u_{t}^{2}(s)\right) d x d s\right\}^{\frac{1}{2}} .
\end{aligned}
$$


Sob essas considerações, para concluir a compacidade assintótica basta verificar que $\Phi_{T}$ é contrativa em $B_{0} \times B_{0}$. Com efeito, seja $\left\{z_{n}\right\}$ uma sequência em $B_{0}$ e $\left\{h_{n}\right\}$ uma sequência em $\mathcal{H}(g)$. Então de (4.10), obtemos que

$$
\left\|U_{h_{n}}(t, 0) z_{n}\right\| \leq C_{T}, \quad \text { para todo } t \in[0, T] .
$$

para alguma constante $C_{T}>0$. Consequentemente $\left\|u_{n}\right\|_{L^{2}\left(0, T ; H^{1}(\Omega)\right)}$ e $\left\|\partial_{t} u_{n}\right\|_{L^{2}\left(0, T ; L^{2}(\Omega)\right)}$ são limitados. Pela imersão compacta de $H^{1}(\Omega)$ em $L^{2}(\Omega)$ e em $L^{4}(\Omega)$, aplicando o Teorema de Aubin-Lions, concluímos que

$$
\begin{array}{lll}
u_{n} \rightarrow u & \text { fortemente em } & L^{2}\left(0, T ; L^{2}(\Omega)\right), \\
u_{n} \rightarrow u & \text { fortemente em } & L^{2}\left(0, T ; L^{4}(\Omega)\right), \\
u_{n} \rightarrow u & \text { fortemente em } & L^{2}\left(0, T ; L^{5}(\Omega)\right) .
\end{array}
$$

Devemos ressaltar que, como $L^{2}\left(\tau_{\epsilon}, t ; L^{2}(\Omega)\right)$ é reflexivo, então

$$
\partial_{t} u_{n_{k}} \rightarrow \partial_{t} u \quad \text { fracamente em } \quad L^{2}\left(0, T ; L^{2}(\Omega)\right)
$$

Note que $\left\{u_{n}\right\}$ tem sequências correspondentes $\left\{z_{n}\right\}$ e $\left\{h_{n}\right\}$. Como $\left\{h_{n}\right\} \subset \mathcal{H}(g)$ e como $\mathcal{H}(g)$ é compacto em $L_{l o c, w}^{2}\left(\mathbb{R} ; L^{2}(\Omega)\right)$, existe $h \in L_{l o c}^{2}\left(\mathbb{R} ; L^{2}(\Omega)\right)$ e uma subsequência, que ainda vamos denotar por $\left\{h_{n}\right\}$, tal que

$$
h_{n} \rightarrow h \quad \text { fracamente em } \quad L_{l o c}^{2}\left(\mathbb{R} ; L^{2}(\Omega)\right)
$$

Como $g$ é regular no tempo, do Corolário 2.4.23, passando a uma subsequência de $\left\{h_{n}\right\}$ se necessário, dado $\eta>0$ existe uma sequência $\left\{\varphi_{n}\right\} \subset H_{b}^{1}\left(\mathbb{R} ; L^{2}(\Omega)\right)$ tal que

$$
\begin{gathered}
\varphi_{n} \rightarrow \varphi, \quad \text { fracamente em } \quad H_{l o c}^{1}\left(\mathbb{R} ; L^{2}(\Omega)\right), \\
\left\|h_{n}-\varphi_{n}\right\|_{L_{b}^{2}\left(\mathbb{R} ; L^{2}(\Omega)\right)}+\|h-\varphi\|_{L_{b}^{2}\left(\mathbb{R} ; L^{2}(\Omega)\right)} \leq \eta .
\end{gathered}
$$

Feito essas observações, para verificar que $\Phi_{T}$ é contrativa, devemos mostrar que

$$
\Phi_{T}\left(z_{n}, z_{m} ; h_{n}, h_{m}\right) \rightarrow 0 \text { quando } n, m \rightarrow \infty
$$


Lembrando que,

$$
\begin{aligned}
\Phi_{T}\left(z_{n}, z_{m} ; h_{n}, h_{m}\right)= & 2\left\{C_{T, 0} \int_{0}^{T}\left\|u_{n}(s)-u_{m}(s)\right\|_{L^{4}(\Omega)}^{2} d s\right\}^{\frac{1}{2}} \\
& +8\left\{\int_{0}^{T} \int_{\Omega}\left(f\left(u_{m}(s)\right)-f\left(u_{n}(s)\right)\right)\left(\partial_{t} u_{n}(s)-\partial_{t} u_{m}(s)\right) d x d s\right\}^{\frac{1}{2}} \\
& +8\left\{\int_{0}^{T} e^{-\sigma_{1}(T-s)} \int_{\Omega}\left(h_{n}(s)-h_{m}(s)\right)\left(u_{n}(s)-u_{m}(s)\right) d x d s\right\}^{\frac{1}{2}} \\
& +8\left\{\int_{0}^{T} e^{-\sigma_{1}(T-s)} \int_{\Omega}\left(h_{n}(s)-h_{m}(s)\right)\left(\partial_{t} u_{n}(s)-\partial_{t} u_{m}(s)\right) d x d s\right\}^{\frac{1}{2}} \\
= & 2\left(I_{1}(n, m)\right)^{\frac{1}{2}}+8\left(I_{2}(n, m)\right)^{\frac{1}{2}}+8\left(I_{3}(n, m)\right)^{\frac{1}{2}}+8\left(I_{4}(n, m)\right)^{\frac{1}{2}},
\end{aligned}
$$

onde $U_{h_{n}}(t, 0) z_{n}=\left(u_{n}(t), \partial_{t} u_{n}(t), \delta_{n}(t), \partial_{t} \delta_{n}(t)\right)$, para todo $t \in[0, T]$.

Para finalizar iremos mostrar que $I_{i}(n, m) \rightarrow 0$ quando $n, m \rightarrow \infty$. Note que $I_{1}(n, m)$ e $I_{2}(n, m)$ são termos similares aos termos da função contrativa $\Psi_{\varepsilon}$ dada por (3.47) e (3.48), logo as demonstrações das convergências são análogas e aqui iremos fazer de um modo resumido.

Começamos provando que $I_{1}(n, m) \rightarrow 0$, para tal, basta notar que, de (4.19), temos que a sequência $\left\{u_{n}\right\}$ é uma sequência de Cauchy em $L^{2}\left(0, T ; L^{4}(\Omega)\right)$ e consequentemente

$$
I_{1}(n, m)=C_{T, 0} \int_{0}^{T}\left\|u_{n}(s)-u_{m}(s)\right\|_{L^{4}(\Omega)}^{2} d s=\left\|u_{n}-u_{m}\right\|_{L^{2}\left(0, T ; L^{4}(\Omega)\right)}^{2} \rightarrow 0,
$$

quando $n, m \rightarrow \infty$.

A parte $I_{2}(n, m)$ de $\Phi_{T}$ envolve os termos de crescimento crítico. Escrevemos

$$
\begin{aligned}
I_{2}(n, m) & =\int_{0}^{T} \int_{\Omega}\left(f\left(u_{m}\right)-f\left(u_{n}\right)\right)\left(\partial_{t} u_{n}-\partial_{t} u_{m}\right) d s \\
& =I_{21}(n, m)+I_{22}(n, m)+I_{23}(n, m),
\end{aligned}
$$

onde

$$
\begin{aligned}
& I_{21}(n, m)=-\int_{\Omega}\left[F\left(u_{m}(T)\right)+F\left(u_{n}(T)\right)\right] d x \\
& I_{22}(n, m)=\int_{\Omega}\left[F\left(u_{m}(0)\right)+F\left(u_{n}(0)\right)\right] d x \\
& I_{23}(n, m)=\int_{0}^{T} \int_{\Omega}\left[f\left(u_{m}(s)\right) \partial_{t} u_{n}(s)+f\left(u_{n}(s)\right) \partial_{t} u_{m}(s)\right] d x d s .
\end{aligned}
$$

Note que, de (4.7), segue que $|F(u)| \leq C\left(1+|u|^{4}\right), u \in \mathbb{R}$ portanto $F$ satisfaz a condição de caratheodory e pelo Teorema 1.0.18 obtemos que o operador Nemytskii 
$F: L^{5}(\Omega) \rightarrow L^{\frac{5}{4}}(\Omega)$ é contínuo. Como $L^{\frac{5}{4}} \hookrightarrow L^{1}$ e $u_{n} \rightarrow u$ fortemente em $L^{2}\left(0, T ; L^{5}(\Omega)\right)$, obtemos que

$$
\begin{gathered}
\left\|F\left(u_{n}\right)(x, T)\right\|_{L^{1}(\Omega)} \rightarrow\|F(u)(x, T)\|_{L^{1}(\Omega)}, \\
\left\|F\left(u_{n}\right)(x, 0)\right\|_{L^{1}(\Omega)} \rightarrow\|F(u)(x, 0)\|_{L^{1}(\Omega)} .
\end{gathered}
$$

Ou seja,

$$
\lim _{n \rightarrow \infty} \lim _{m \rightarrow \infty}\left[I_{21}(n, m)+I_{22}(n, m)\right]=-2 \int_{\Omega} F(u(T)) d x+2 \int_{\Omega} F(u(0)) d x .
$$

Por outro lado, como $|f(u)| \leq C\left(1+|u|^{3}\right)$, obtemos que a não linearidade $f$ satisfaz a condição de caratheodory e pelo Teorema 1.0.18 obtemos que o operador Nemytskii $N_{f}: L^{6}(\Omega) \rightarrow L^{2}(\Omega)$ leva conjuntos limitados em conjuntos limitados. Agora, defina

$$
\mathbb{G}_{m}(s)=\int_{\Omega} f\left(u_{m}(s)\right) \partial_{t} u_{n}(s) d x \quad \text { e } \mathbb{G}(s)=\int_{\Omega} f(u(s)) \partial_{t} u_{n}(s) d x .
$$

Observe que

$$
\left|\mathbb{G}_{m}(s)\right| \leq\left(\int_{\Omega}\left|f\left(u_{m}(s)\right)\right|^{2} d x\right)^{\frac{1}{2}}\left(\int_{\Omega}\left|\partial_{t} u_{n}(s)\right|^{2} d x\right)^{\frac{1}{2}}
$$

Logo, usando que o operador $N_{f}$ leva limitados em limitados e o fato de que $\left\|\partial_{t} u_{n}\right\|_{L^{2}\left(0, T ; L^{2}(\Omega)\right)}$ é limitado em $\mathbb{R}$, obtemos que

$$
\left|\mathbb{G}_{m}(s)\right| \leq M, \quad \text { quase sempre em } \quad[0, T]
$$

Provemos que $\mathbb{G}_{m}(s) \rightarrow \mathbb{G}(s)$ quase sempre em $[0, T]$ quando $m \rightarrow \infty$. De fato:

$$
\left|\mathbb{G}_{m}(s)-\mathbb{G}(s)\right| \leq \int_{\Omega}\left|f\left(u_{m}(s)\right)-f(u(s))\right|\left|\partial_{t} u_{n}(s)\right| d x \rightarrow 0,
$$

quase sempre em $[0, T]$ e quando $m \rightarrow \infty$. Portanto pelo teorema da convergência dominada,

$$
\int_{0}^{T} \mathbb{G}_{m}(s) d s \rightarrow \int_{0}^{T} \mathbb{G}(s) d s \text { quando } \quad m \rightarrow \infty
$$

Assim, pelo fato de que $\partial_{t} u_{n} \rightarrow \partial_{t} u$ em $L^{2}\left(0, T ; L^{2}(\Omega)\right)$, não é difícil de ver que

$$
\begin{aligned}
\lim _{n \rightarrow \infty}\left[\lim _{m \rightarrow \infty} \int_{0}^{T} \int_{\Omega} f\left(u_{m}\right) \partial_{t} u_{n} d x d s\right] & =\int_{0}^{T} \int_{\Omega} f(u) \partial_{t} u d x d s \\
& =\lim _{m \rightarrow \infty}\left[\lim _{n \rightarrow \infty} \int_{0}^{T} \int_{\Omega} f\left(u_{m}\right) \partial_{t} u_{n} d x d s\right] .
\end{aligned}
$$


Consequentemente,

$$
\begin{aligned}
\lim _{n \rightarrow \infty}\left[\lim _{m \rightarrow \infty} I_{23}(n, m)\right] & =2 \int_{0}^{T} \int_{\Omega} f(u(s)) \partial_{t} u(s) d x d s \\
& =2 \int_{\Omega} F(u(T)) d x-2 \int_{\Omega} F(u(0)) d x .
\end{aligned}
$$

Então

$$
\lim _{n \rightarrow \infty} \lim _{m \rightarrow \infty} \int_{0}^{T} \int_{\Omega}\left(f\left(u_{m}\right)-f\left(u_{n}\right)\right)\left(\partial_{t} u_{n}-\partial_{t} u_{m}\right) d s=0 .
$$

Isso prova que

$$
\lim _{n \rightarrow \infty} \lim _{m \rightarrow \infty} I_{2}(n, m)=0 .
$$

A parte $I_{3}(n, m)$ de $\Phi_{T}$ envolve termos relacionados com a envoltória fraca $\mathcal{H}(g)$ onde $g$ é a força externa inicial regular no tempo. Escreva

$$
\begin{aligned}
I_{3}(m, n) & =\int_{0}^{T} e^{-\sigma_{1}(T-s)} \int_{\Omega}\left(h_{n}(s)-h_{m}(s)\right)\left(u_{n}(s)-u_{m}(s)\right) d x d s \\
& =I_{31}(n, m)+I_{32}(n, m)+I_{33}(n, m)+I_{34}(n, m),
\end{aligned}
$$

onde

$$
\begin{aligned}
& I_{31}(n, m)=\int_{0}^{T} e^{-\sigma_{1}(T-s)} \int_{\Omega} h_{n}(s) u_{n}(s) d x d s, \\
& I_{32}(n, m)=-\int_{0}^{T} e^{-\sigma_{1}(T-s)} \int_{\Omega} h_{n}(s) u_{m}(s) d x d s \\
& I_{33}(n, m)=-\int_{0}^{T} e^{-\sigma_{1}(T-s)} \int_{\Omega} h_{m}(s) u_{n}(s) d x d s, \\
& I_{34}(n, m)=\int_{0}^{T} e^{-\sigma_{1}(T-s)} \int_{\Omega} h_{m}(s) u_{m}(s) d x d s .
\end{aligned}
$$

Começamos analisando $I_{31}(n, m)$. Note que

$$
\begin{aligned}
\int_{0}^{T} & e^{-\sigma_{1}(T-s)} \int_{\Omega}\left[h_{n}(s) u_{n}(s)-h(s) u(s)\right] d x d s \\
& \leq \int_{0}^{T} \int_{\Omega}\left[h_{n}(s) u_{n}(s)-h_{n}(s) u(s)+h_{n}(s) u(s)-h(s) u(s)\right] d x d s \\
& =\int_{0}^{T} \int_{\Omega} h_{n}(s)\left[u_{n}(s)-u(s)\right] d x d s+\int_{0}^{T} \int_{\Omega}\left[h_{n}(s)-h(s)\right] u(s) d x d s .
\end{aligned}
$$

Observe que da convergência fraca (4.21), que

$$
\left\langle G, h_{n}\right\rangle \rightarrow\langle G, h\rangle, \quad \text { para todo } \quad G \in\left[L^{2}\left(0, T ; L^{2}(\Omega)\right)\right]^{\prime},
$$

e que $\left\{h_{n}\right\}$ é uma sequência limitada, isto é,

$$
\left\|h_{n}\right\|_{L^{2}\left(0, T ; L^{2}(\Omega)\right)} \leq M
$$


Em particular, defina

$$
\begin{aligned}
G_{u}: L^{2}\left(0, T ; L^{2}(\Omega)\right) & \longrightarrow \mathbb{R} \\
\xi & \longrightarrow\left\langle G_{u}, \xi\right\rangle=\int_{0}^{T} \int_{\Omega} \xi(s) u(s) d x d s .
\end{aligned}
$$

É fácil ver que $G_{u}$ é linear e, da desigualdade de Holder,

$$
\left|\left\langle G_{u}, \xi\right\rangle\right| \leq\|u\|_{L^{2}\left(0, T ; L^{2}(\Omega)\right)}\|\xi\|_{L^{2}\left(0, T ; L^{2}(\Omega)\right)},
$$

isto é, $G_{u} \in\left[L^{2}\left(0, T ; L^{2}(\Omega)\right)\right]^{\prime}$. Portanto

$$
\left|\int_{0}^{T} \int_{\Omega} h_{n}(s) u(s) d x d s-\int_{0}^{T} \int_{\Omega} h(s) u(s) d x d s\right| \rightarrow 0 \quad \text { quando } \quad n \rightarrow \infty .
$$

Voltando em (4.26), concluímos que

$$
\begin{aligned}
& \left|\int_{0}^{T} e^{-\sigma_{1}(T-s)} \int_{\Omega}\left[h_{n}(s) u_{n}(s)-h(s) u(s)\right] d x d s\right| \\
& \quad \leq\left|\int_{0}^{T} \int_{\Omega} h_{n}(s)\left[u_{n}(s)-u(s)\right] d x d s+\int_{0}^{T} \int_{\Omega}\left[h_{n}(s)-h(s)\right] u(s) d x d s\right| \\
& \quad \leq \int_{0}^{T}\left\|h_{n}(s)\right\|_{L^{2}(\Omega)}\left\|u_{n}(s)-u(s)\right\|_{L^{2}(\Omega)} d s+\left|\int_{0}^{T} \int_{\Omega}\left[h_{n}(s)-h(s)\right] u(s) d x d s\right| \\
& \quad \leq\left\|h_{n}\right\|_{L^{2}\left(0, T ; L^{2}(\Omega)\right)}\left\|u_{n}-u\right\|_{L^{2}\left(0, T ; L^{2}(\Omega)\right)}+\left|\int_{0}^{T} \int_{\Omega}\left[h_{n}(s)-h(s)\right] u(s) d x d s\right| .
\end{aligned}
$$

Assim, de (4.18), (4.27) e (4.28), segue que

$$
I_{31}(m, n)=\int_{0}^{T} e^{-\sigma_{1}(T-s)} \int_{\Omega} h_{n}(s) u_{n}(s) d x d s \rightarrow \int_{0}^{T} e^{-\sigma_{1}(T-s)} \int_{\Omega} h(s) u(s) d x d s,
$$

quando $n \rightarrow \infty$. Analogamente

$$
I_{34}(n, m)=\int_{0}^{T} e^{-\sigma_{1}(T-s)} \int_{\Omega} h_{m}(s) u_{m}(s) d x d s \rightarrow \int_{0}^{T} e^{-\sigma_{1}(T-s)} \int_{\Omega} h(s) u(s) d x d s,
$$

quando $m \rightarrow \infty$. Agora vamos analisar $I_{32}(n, m)$, para tal, primeiro vamos fazer $m$ fixo e $n \rightarrow \infty$, em seguida fazer $m \rightarrow \infty$. Então, para cada $m$ fixo, defina

$$
\begin{aligned}
G_{u_{m}}: L^{2}\left(0, T ; L^{2}(\Omega)\right) & \rightarrow \mathbb{R} \\
\xi & \rightarrow\left\langle G_{u_{m}}, \xi\right\rangle=\int_{0}^{T} \int_{\Omega} \xi(s) u_{m}(s) d x d s .
\end{aligned}
$$

Claramente $G_{u_{m}} \in\left[L^{2}\left(0, T ; L^{2}(\Omega)\right)\right]^{\prime}$ para cada $m$. Da convergência fraca (4.21), para cada $m$, temos que

$$
I_{32}(n, m)=-\int_{0}^{T} e^{-\sigma_{1}(T-s)} \int_{\Omega} h_{n}(s) u_{m}(s) d x d s \rightarrow-\int_{0}^{T} e^{-\sigma_{1}(T-s)} \int_{\Omega} h(s) u_{m}(s) d x d s,
$$


quando $n \rightarrow \infty$. Da convergência forte (4.18), tem-se

$$
-\int_{0}^{T} e^{-\sigma_{1}(T-s)} \int_{\Omega} h(s) u_{m}(s) d x d s \rightarrow-\int_{0}^{T} e^{-\sigma_{1}(T-s)} \int_{\Omega} h(s) u(s) d x d s,
$$

quando $m \rightarrow \infty$. Portanto

$$
\lim _{m \rightarrow \infty}\left[\lim _{n \rightarrow \infty} I_{32}(n, m)\right]=-\int_{0}^{T} e^{-\sigma_{1}(T-s)} \int_{\Omega} h(s) u(s) d x d s .
$$

Analogamente, olhando para $I_{33}(n, m)$,

$$
\lim _{n \rightarrow \infty}\left[\lim _{m \rightarrow \infty} I_{33}(n, m)\right]=-\int_{0}^{T} e^{-\sigma_{1}(T-s)} \int_{\Omega} h(s) u(s) d x d s .
$$

Combinando essas informações, chegamos na seguinte conclusão

$$
\begin{aligned}
\lim _{n \rightarrow \infty} \lim _{m \rightarrow \infty} & I_{3}(n, m) \\
& =\lim _{n \rightarrow \infty} \lim _{m \rightarrow \infty}\left[I_{31}(n, m)+I_{32}(n, m)+I_{33}(n, m)+I_{34}(n, m)\right]=0 .
\end{aligned}
$$

Resta verificar que

$$
\lim _{n \rightarrow \infty} \lim _{m \rightarrow \infty} I_{4}(n, m)=0 .
$$

De fato, analisando $I_{4}(n, m)$, vemos que

$$
\begin{aligned}
I_{4}(n, m)= & \int_{0}^{T} e^{-\sigma_{1}(T-s)} \int_{\Omega}\left(h_{n}(s)-h_{m}(s)\right)\left(\partial_{t} u_{n}(s)-\partial_{t} u_{m}(s)\right) d x d s \\
= & \int_{0}^{T} e^{-\sigma_{1}(T-s)} \int_{\Omega}\left(h_{n}(s) \partial_{t} u_{n}(s)-h_{n}(s) \partial_{t} u_{m}(s)\right. \\
& \left.-h_{m}(s) \partial_{t} u_{n}(s)+h_{m}(s) \partial_{t} u_{m}(s)\right) d x d s \\
= & I_{41}(n, m)+I_{42}(n, m)+I_{43}(n, m)+I_{44}(n, m),
\end{aligned}
$$

onde

$$
\begin{aligned}
& I_{41}(n, m)=\int_{0}^{T} e^{-\sigma_{1}(T-s)} \int_{\Omega} h_{n}(s) \partial_{t} u_{n}(s) d x d s \\
& I_{42}(n, m)=-\int_{0}^{T} e^{-\sigma_{1}(T-s)} \int_{\Omega} h_{n}(s) \partial_{t} u_{m}(s) d x d s \\
& I_{43}(n, m)=-\int_{0}^{T} e^{-\sigma_{1}(T-s)} \int_{\Omega} h_{m}(s) \partial_{t} u_{n}(s) d x d s \\
& I_{44}(n, m)=\int_{0}^{T} e^{-\sigma_{1}(T-s)} \int_{\Omega} h_{m}(s) \partial_{t} u_{m}(s) d x d s .
\end{aligned}
$$


Olhando para $I_{41}(n, m)$, observe que

$$
\begin{aligned}
& \int_{0}^{T} e^{-\sigma_{1}(T-s)} \int_{\Omega}\left(h_{n}(s) \partial_{t} u_{n}(s)-h(s) \partial_{t} u(s)\right) d x d s \\
&=\int_{0}^{T} e^{-\sigma_{1}(T-s)} \int_{\Omega}\left(h_{n}(s) \partial_{t} u_{n}(s)-\varphi_{n}(s) \partial_{t} u_{n}(s)+\varphi_{n}(s) \partial_{t} u_{n}(s)\right. \\
&\left.\quad-\varphi(s) \partial_{t} u(s)+\varphi(s) \partial_{t} u(s)-h(s) \partial_{t} u(s)\right) d x d s .
\end{aligned}
$$

Provemos que

$$
\int_{0}^{T} e^{-\sigma_{1}(T-s)} \int_{\Omega} \varphi_{n}(s) \partial_{t} u_{n}(s) d x d s \rightarrow \int_{0}^{T} e^{-\sigma_{1}(T-s)} \int_{\Omega} \varphi(s) \partial_{t} u(s) d x d s,
$$

Integrando por partes

$$
\begin{aligned}
\int_{0}^{T} e^{-\sigma_{1}(T-s)} \int_{\Omega} \varphi_{n}(s) \partial_{t} u_{n}(s) & d x d s=\int_{0}^{T} \int_{\Omega} \sigma_{1} e^{-\sigma_{1}(T-s)} \varphi_{n}(s) u_{n}(s) d x d s \\
+ & \int_{0}^{T} \int_{\Omega} e^{-\sigma_{1}(T-s)} \partial_{t} \varphi_{n}(s) u_{n}(s) d x d s \\
& +\int_{\Omega} \varphi_{n}(T) u_{n}(T) d x-\int_{\Omega} e^{-\sigma_{1} T} \varphi_{n}(0) u_{n}(0) d x
\end{aligned}
$$

Note que

$$
\begin{aligned}
\int_{0}^{T} & \sigma_{1} e^{-\sigma_{1}(T-s)} \int_{\Omega}\left[\varphi_{n}(s) u_{n}(s)-\varphi(s) u(s)\right] d x d s \\
& \leq \int_{0}^{T} \int_{\Omega} \sigma_{1}\left[\varphi_{n}(s) u_{n}(s)-\varphi_{n}(s) u(s)+\varphi_{n}(s) u(s)-\varphi(s) u(s)\right] d x d s \\
& =\int_{0}^{T} \int_{\Omega} \sigma_{1} \varphi_{n}(s)\left[u_{n}(s)-u(s)\right] d x d s+\int_{0}^{T} \int_{\Omega} \sigma_{1}\left[\varphi_{n}(s)-\varphi(s)\right] u(s) d x d s .
\end{aligned}
$$

Da convergência fraca (4.22), segue que

$$
\left|\int_{0}^{T} \int_{\Omega} \sigma_{1}\left[\varphi_{n}(s)-\varphi(s)\right] u(s) d x d s\right|=\left|\left\langle G_{\sigma_{1} u}, \varphi_{n}\right\rangle-\left\langle G_{\sigma_{1} u}, \varphi\right\rangle\right| \rightarrow 0,
$$

onde $G_{\sigma_{1} u} \in\left[L^{2}\left(0, T ; L^{2}(\Omega)\right)\right]^{\prime}$ é dada por

$$
\left\langle G_{\sigma_{1} u}, \xi\right\rangle=\int_{0}^{T} \int_{\Omega} \sigma_{1} u(s) \xi(s) d x d s \quad \text { para todo } \quad \xi \in L^{2}\left(0, T ; L^{2}(\Omega)\right) .
$$

Logo, como $\varphi_{n} \rightarrow \varphi$ em $L^{2}\left(0, T ; L^{2}(\Omega)\right)$, então $\left\{\varphi_{n}\right\}$ é limitada em $L^{2}\left(0, T ; L^{2}(\Omega)\right)$ e

$$
\begin{aligned}
& \left|\int_{0}^{T} \sigma_{1} e^{-\sigma_{1}(T-s)} \int_{\Omega}\left[\varphi_{n}(s) u_{n}(s)-\varphi(s) u(s)\right] d x d s\right| \\
& \quad \leq \sigma_{1}\left\|\varphi_{n}\right\|_{L^{2}\left(0, T ; L^{2}(\Omega)\right)}\left\|u_{n}-u\right\|_{L^{2}\left(0, T ; L^{2}(\Omega)\right)}+\left|\left\langle G_{\sigma_{1} u}, \varphi_{n}\right\rangle-\left\langle G_{\sigma_{1} u}, \varphi\right\rangle\right| \rightarrow 0,
\end{aligned}
$$


quando $n \rightarrow \infty$, isto é,

$$
(4.31) \rightarrow \int_{0}^{T} \sigma_{1} e^{-\sigma_{1}(T-s)} \int_{\Omega} \varphi(s) u(s) d x d s .
$$

Analogamente, de 4.22 , temos que $\partial_{t} \varphi_{n} \rightarrow \partial_{t} \varphi$ em $L^{2}\left(0, T ; L^{2}(\Omega)\right)$ e

$$
\begin{aligned}
& \left|\int_{0}^{T} e^{-\sigma_{1}(T-s)} \int_{\Omega}\left[\partial_{t} \varphi_{n}(s) u_{n}(s)-\partial_{t} \varphi(s) u(s)\right] d x d s\right| \\
& \quad \leq\left\|\partial_{t} \varphi_{n}\right\|_{L^{2}\left(0, T ; L^{2}(\Omega)\right)}\left\|u_{n}-u\right\|_{L^{2}\left(0, T ; L^{2}(\Omega)\right)}+\left|\left\langle G_{u}, \partial_{t} \varphi_{n}\right\rangle-\left\langle G_{u}, \partial_{t} \varphi\right\rangle\right| \rightarrow 0,
\end{aligned}
$$

quando $n \rightarrow \infty$, ou seja,

$$
(4.32) \rightarrow \int_{0}^{T} e^{-\sigma_{1}(T-s)} \int_{\Omega} \partial_{t} \varphi(s) u(s) d x d s .
$$

Veja que, de (4.18) e de (4.22), temos

$$
\begin{array}{ccc}
u_{n}(T) \rightarrow u(T) & \text { fortemente em } & L^{2}(\Omega), \\
u_{n}(0) \rightarrow u(0) & \text { fortemente em } & L^{2}(\Omega), \\
\varphi_{n}(T) \rightarrow \varphi(T) & \text { fracamente em } & L^{2}(\Omega), \\
\varphi_{n}(0) \rightarrow \varphi(0) & \text { fracamente em } & L^{2}(\Omega) .
\end{array}
$$

Portanto, similarmente ao que já foi feito,

$$
(4.33) \rightarrow \int_{\Omega} \varphi(T) u(T) d x-\int_{\Omega} e^{-\sigma_{1} T} \varphi(0) u(0) d x .
$$

Combinando (4.34), (4.35) e (4.36), concluímos que

$$
\begin{aligned}
\int_{0}^{T} e^{-\sigma_{1}(T-s)} \int_{\Omega} \varphi_{n}(s) \partial_{t} u_{n}(s) d x d s= & \int_{0}^{T} \int_{\Omega} \sigma_{1} e^{-\sigma_{1}(T-s)} \varphi_{n}(s) u_{n}(s) d x d s \\
& +\int_{0}^{T} \int_{\Omega} e^{-\sigma_{1}(T-s)} \partial_{t} \varphi_{n}(s) u_{n}(s) d x d s \\
& +\int_{\Omega} \varphi_{n}(T) u_{n}(T) d x-\int_{\Omega} e^{-\sigma_{1} T} \varphi_{n}(0) u_{n}(0) d x \\
\int_{0}^{T} e^{-\sigma_{1}(T-s)} \int_{\Omega} \varphi_{n}(s) \partial_{t} u_{n}(s) d x d s \rightarrow & \int_{\Omega}^{T} \sigma_{1} e^{-\sigma_{1}(T-s)} \varphi(s) u(s) d x d s \\
& +\int_{0}^{T} \int_{\Omega} e^{-\sigma_{1}(T-s)} \partial_{t} \varphi(s) u(s) d x d s \\
& +\int_{\Omega} \varphi(T) u(T) d x-\int_{\Omega} e^{-\sigma_{1} T} \varphi(0) u(0) d x \\
= & \int_{0}^{T} e^{-\sigma_{1}(T-s)} \int_{\Omega} \varphi(s) \partial_{t} u(s) d x d s .
\end{aligned}
$$


Isto é,

$$
\int_{0}^{T} e^{-\sigma_{1}(T-s)} \int_{\Omega} \varphi_{n}(s) \partial_{t} u_{n}(s) d x d s \rightarrow \int_{0}^{T} e^{-\sigma_{1}(T-s)} \int_{\Omega} \varphi(s) \partial_{t} u(s) d x d s .
$$

Voltando em (4.30), temos que

$$
\begin{aligned}
& \left|\int_{0}^{T} e^{-\sigma_{1}(T-s)} \int_{\Omega}\left(h_{n}(s) \partial_{t} u_{n}(s)-h(s) \partial_{t} u(s)\right) d x d s\right| \\
& \leq \int_{0}^{T} e^{-\sigma_{1}(T-s)} \int_{\Omega}\left|h_{n}(s) \partial_{t} u_{n}(s)-\varphi_{n}(s) \partial_{t} u_{n}(s)\right| d x d s \\
& +\int_{0}^{T} e^{-\sigma_{1}(T-s)} \int_{\Omega}\left|\varphi_{n}(s) \partial_{t} u_{n}(s)-\varphi(s) \partial_{t} u(s)\right| d x d s \\
& +\int_{0}^{T} e^{-\sigma_{1}(T-s)} \int_{\Omega}\left|\varphi(s) \partial_{t} u(s)-h(s) \partial_{t} u(s)\right| d x d s \\
& \leq\left(\int_{0}^{T}\left\|\partial_{t} u_{n}(s)\right\|_{L^{2}(\Omega)}^{2} d s\right)^{\frac{1}{2}}\left(\int_{0}^{T} e^{-2 \sigma_{1}(T-s)}\left\|h_{n}(s)-\varphi_{n}(s)\right\|_{L^{2}(\Omega)}^{2} d s\right)^{\frac{1}{2}} \\
& +\int_{0}^{T} e^{-\sigma_{1}(T-s)} \int_{\Omega}\left|\varphi_{n}(s) \partial_{t} u_{n}(s)-\varphi(s) \partial_{t} u(s)\right| d x d s \\
& +\left(\int_{0}^{T}\left\|\partial_{t} u(s)\right\|_{L^{2}(\Omega)}^{2} d s\right)^{\frac{1}{2}}\left(\int_{0}^{T} e^{-2 \sigma_{1}(T-s)}\|h(s)-\varphi(s)\|_{L^{2}(\Omega)}^{2} d s\right)^{\frac{1}{2}} \\
& \leq \frac{C_{T}}{1-e^{-2 \sigma_{1}}}\left(\sup _{t \in \mathbb{R}} \int_{t}^{t+1}\left\|h_{n}(s)-\varphi_{n}(s)\right\|_{L^{2}(\Omega)}^{2} d s\right)^{\frac{1}{2}} \\
& +\frac{C_{T}}{1-e^{-2 \sigma_{1}}}\left(\sup _{t \in \mathbb{R}} \int_{t}^{t+1}\|h(s)-\varphi(s)\|_{L^{2}(\Omega)}^{2} d s\right)^{\frac{1}{2}} \\
& +\int_{0}^{T} e^{-\sigma_{1}(T-s)} \int_{\Omega}\left|\varphi_{n}(s) \partial_{t} u_{n}(s)-\varphi(s) \partial_{t} u(s)\right| d x d s \\
& =C_{T}\left(\left\|h_{n}-\varphi_{n}\right\|_{L_{b}^{2}\left(\mathbb{R}, L^{2}(\Omega)\right)}+\|h-\varphi\|_{L_{b}^{2}\left(\mathbb{R}, L^{2}(\Omega)\right)}\right) \\
& +\int_{0}^{T} e^{-\sigma_{1}(T-s)} \int_{\Omega}\left|\varphi_{n}(s) \partial_{t} u_{n}(s)-\varphi(s) \partial_{t} u(s)\right| d x d s .
\end{aligned}
$$

Assim, de (4.23), segue que

$$
\begin{aligned}
\mid \int_{0}^{T} e^{-\sigma_{1}(T-s)} & \int_{\Omega}\left(h_{n}(s) \partial_{t} u_{n}(s)-h(s) \partial_{t} u(s)\right) d x d s \mid \\
& \leq \frac{C_{T}}{1-e^{-2 \sigma_{1}}} \eta+\int_{0}^{T} e^{-\sigma_{1}(T-s)} \int_{\Omega}\left|\varphi_{n}(s) \partial_{t} u_{n}(s)-\varphi(s) \partial_{t} u(s)\right| d x d s
\end{aligned}
$$

para todo $n \in \mathbb{N}$. Pela arbitrariedade do $\eta>0$ e de (4.37)

$$
\begin{aligned}
\lim _{n \rightarrow \infty} \lim _{m \rightarrow \infty} I_{41}(m, n) & =\lim _{n \rightarrow \infty} \lim _{m \rightarrow \infty}\left[\int_{0}^{T} e^{-\sigma_{1}(T-s)} \int_{\Omega} h_{n}(s) \partial_{t} u_{n}(s) d x d s\right] \\
& =\int_{0}^{T} e^{-\sigma_{1}(T-s)} \int_{\Omega} h(s) \partial_{t} u(s) d x d s .
\end{aligned}
$$


Analogamente

$$
\begin{aligned}
\lim _{n \rightarrow \infty} \lim _{m \rightarrow \infty} I_{44}(m, n) & =\lim _{n \rightarrow \infty} \lim _{m \rightarrow \infty}\left[\int_{0}^{T} e^{-\sigma_{1}(T-s)} \int_{\Omega} h_{m}(s) \partial_{t} u_{m}(s) d x d s\right] \\
& =\int_{0}^{T} e^{-\sigma_{1}(T-s)} \int_{\Omega} h(s) \partial_{t} u(s) d x d s
\end{aligned}
$$

Agora, olhando para $I_{42}(n, m)$, observe que

$$
\begin{aligned}
\int_{0}^{T} e^{-\sigma_{1}(T-s)} \int_{\Omega}\left(h_{n}(s) \partial_{t} u_{m}(s)-h(s) \partial_{t} u(s)\right) d x d s & \\
= & \int_{0}^{T} e^{-\sigma_{1}(T-s)} \int_{\Omega}\left(h_{n}(s) \partial_{t} u_{m}(s)-\varphi_{n}(s) \partial_{t} u_{m}(s)+\varphi_{n}(s) \partial_{t} u_{m}(s)\right. \\
& \left.\quad-\varphi(s) \partial_{t} u(s)+\varphi(s) \partial_{t} u(s)-h(s) \partial_{t} u(s)\right) d x d s .
\end{aligned}
$$

Provemos que

$$
\int_{0}^{T} e^{-\sigma_{1}(T-s)} \int_{\Omega} \varphi_{n}(s) \partial_{t} u_{m}(s) d x d s \rightarrow \int_{0}^{T} e^{-\sigma_{1}(T-s)} \int_{\Omega} \varphi(s) \partial_{t} u(s) d x d s .
$$

Integrando por partes

$$
\begin{aligned}
\int_{0}^{T} e^{-\sigma_{1}(T-s)} \int_{\Omega} \varphi_{n}(s) \partial_{t} u_{m} & (s) d x d s=\int_{0}^{T} \int_{\Omega} \sigma_{1} e^{-\sigma_{1}(T-s)} \varphi_{n}(s) u_{m}(s) d x d s \\
& +\int_{0}^{T} \int_{\Omega} e^{-\sigma_{1}(T-s)} \partial_{t} \varphi_{n}(s) u_{m}(s) d x d s \\
& +\int_{\Omega} \varphi_{n}(T) u_{m}(T) d x-\int_{\Omega} e^{-\sigma_{1} T} \varphi_{n}(0) u_{m}(0) d x
\end{aligned}
$$

Como $\varphi_{n} \rightarrow \varphi$ em $H^{1}\left(0, T ; L^{2}(\Omega)\right)$ e $u_{n} \rightarrow u$ em $L^{2}\left(0, T ; L^{2}(\Omega)\right)$, temos, primeiro fixando $m \in \mathbb{N}$ e fazendo $n \rightarrow \infty$, em seguida fazendo $m \rightarrow \infty$, que

$$
\begin{aligned}
& \lim _{m \rightarrow \infty}\left[\lim _{n \rightarrow \infty}(4.42)\right]=\lim _{m \rightarrow \infty} \int_{0}^{T} \int_{\Omega} \sigma_{1} e^{-\sigma_{1}(T-s)} \varphi(s) u_{m}(s) d x d s \\
&=\int_{0}^{T} \int_{\Omega} \sigma_{1} e^{-\sigma_{1}(T-s)} \varphi(s) u(s) d x d s \\
& \lim _{m \rightarrow \infty}\left[\lim _{n \rightarrow \infty}(4.43)\right]=\lim _{m \rightarrow \infty} \int_{0}^{T} \int_{\Omega} e^{-\sigma_{1}(T-s)} \partial_{t} \varphi(s) u_{m}(s) d x d s \\
&=\int_{0}^{T} \int_{\Omega} e^{-\sigma_{1}(T-s)} \partial_{t} \varphi(s) u(s) d x d s \\
& \lim _{m \rightarrow \infty}\left[\lim _{n \rightarrow \infty}(4.44)\right]=\lim _{m \rightarrow \infty}\left[\int_{\Omega} \varphi(T) u_{m}(T) d x d s+\int_{\Omega} e^{-\sigma_{1} T} \varphi(0) u_{m}(0) d x d s\right] \\
&=\int_{\Omega} \varphi(T) u(T) d x d s+\int_{\Omega} e^{-\sigma_{1} T} \varphi(0) u(0) d x d s .
\end{aligned}
$$


Assim, vale a convergência (4.41) quando $n, m \rightarrow \infty$. Voltando em (4.40), temos que

$$
\begin{aligned}
& \left|\int_{0}^{T} e^{-\sigma_{1}(T-s)} \int_{\Omega}\left(h_{n}(s) \partial_{t} u_{m}(s)-h(s) \partial_{t} u(s)\right) d x d s\right| \\
& \leq \int_{0}^{T} e^{-\sigma_{1}(T-s)} \int_{\Omega}\left|h_{n}(s) \partial_{t} u_{m}(s)-\varphi_{n}(s) \partial_{t} u_{m}(s)\right| d x d s \\
& +\int_{0}^{T} e^{-\sigma_{1}(T-s)} \int_{\Omega}\left|\varphi_{n}(s) \partial_{t} u_{m}(s)-\varphi(s) \partial_{t} u(s)\right| d x d s \\
& +\int_{0}^{T} e^{-\sigma_{1}(T-s)} \int_{\Omega}\left|\varphi(s) \partial_{t} u(s)-h(s) \partial_{t} u(s)\right| d x d s \\
& \leq\left(\int_{0}^{T}\left\|\partial_{t} u_{m}(s)\right\|_{L^{2}(\Omega)}^{2} d s\right)^{\frac{1}{2}}\left(\int_{0}^{T} e^{-2 \sigma_{1}(T-s)}\left\|h_{n}(s)-\varphi_{n}(s)\right\|_{L^{2}(\Omega)}^{2} d s\right)^{\frac{1}{2}} \\
& +\int_{0}^{T} e^{-\sigma_{1}(T-s)} \int_{\Omega}\left|\varphi_{n}(s) \partial_{t} u_{m}(s)-\varphi(s) \partial_{t} u(s)\right| d x d s \\
& +\left(\int_{0}^{T}\left\|\partial_{t} u(s)\right\|_{L^{2}(\Omega)}^{2} d s\right)^{\frac{1}{2}}\left(\int_{0}^{T} e^{-2 \sigma_{1}(T-s)}\|h(s)-\varphi(s)\|_{L^{2}(\Omega)}^{2} d s\right)^{\frac{1}{2}} \\
& \leq \frac{C_{T}}{1-e^{-2 \sigma_{1}}}\left(\sup _{t \in \mathbb{R}} \int_{t}^{t+1}\left\|h_{n}(s)-\varphi_{n}(s)\right\|_{L^{2}(\Omega)}^{2} d s\right)^{\frac{1}{2}} \\
& +\frac{C_{T}}{1-e^{-2 \sigma_{1}}}\left(\sup _{t \in \mathbb{R}} \int_{t}^{t+1}\|h(s)-\varphi(s)\|_{L^{2}(\Omega)}^{2} d s\right)^{\frac{1}{2}} \\
& +\int_{0}^{T} e^{-\sigma_{1}(T-s)} \int_{\Omega}\left|\varphi_{n}(s) \partial_{t} u_{m}(s)-\varphi(s) \partial_{t} u(s)\right| d x d s \\
& =C_{T}\left(\left\|h_{n}-\varphi_{n}\right\|_{L_{b}^{2}\left(\mathbb{R}, L^{2}(\Omega)\right)}+\|h-\varphi\|_{L_{b}^{2}\left(\mathbb{R}, L^{2}(\Omega)\right)}\right) \\
& +\int_{0}^{T} e^{-\sigma_{1}(T-s)} \int_{\Omega}\left|\varphi_{n}(s) \partial_{t} u_{m}(s)-\varphi(s) \partial_{t} u(s)\right| d x d s .
\end{aligned}
$$

Assim, de (4.23), obtemos

$$
\begin{aligned}
& \left|\int_{0}^{T} e^{-\sigma_{1}(T-s)} \int_{\Omega}\left(h_{n}(s) \partial_{t} u_{n}(s)-h(s) \partial_{t} u(s)\right) d x d s\right| \\
& \quad \leq \frac{C_{T}}{1-e^{-2 \sigma_{1}}} \eta+\int_{0}^{T} e^{-\sigma_{1}(T-s)} \int_{\Omega}\left|\varphi_{n}(s) \partial_{t} u_{n}(s)-\varphi(s) \partial_{t} u(s)\right| d x d s
\end{aligned}
$$

para todo $n \in \mathbb{N}$. Pela arbitrariedade do $\eta>0$ e de (4.41)

$$
\begin{aligned}
\lim _{n \rightarrow \infty} \lim _{m \rightarrow \infty} I_{42}(m, n) & =\lim _{m \rightarrow \infty}\left[\lim _{n \rightarrow \infty}-\int_{0}^{T} e^{-\sigma_{1}(T-s)} \int_{\Omega} h_{n}(s) \partial_{t} u_{m}(s) d x d s\right] \\
& =-\int_{0}^{T} e^{-\sigma_{1}(T-s)} \int_{\Omega} h(s) \partial_{t} u(s) d x d s
\end{aligned}
$$


Analogamente

$$
\begin{aligned}
\lim _{n \rightarrow \infty} \lim _{m \rightarrow \infty} I_{43}(m, n) & =\lim _{n \rightarrow \infty}\left[\lim _{m \rightarrow \infty}-\int_{0}^{T} e^{-\sigma_{1}(T-s)} \int_{\Omega} h_{m}(s) \partial_{t} u_{n}(s) d x d s\right] \\
& =-\int_{0}^{T} e^{-\sigma_{1}(T-s)} \int_{\Omega} h(s) \partial_{t} u(s) d x d s .
\end{aligned}
$$

Combinando (4.38), (4.39), (4.45) e (4.46), chegamos em

$$
\begin{aligned}
\lim _{n \rightarrow \infty} & \lim _{m \rightarrow \infty} I_{4}(m, n) \\
\quad & =\lim _{n \rightarrow \infty} \lim _{m \rightarrow \infty}\left[I_{41}(m, n)+I_{42}(m, n)+I_{43}(m, n)+I_{44}(m, n)\right]=0 .
\end{aligned}
$$

Finalmente, dos limites (4.24), (4.25), (4.29) e (4.47), segue que

$$
\lim _{n \rightarrow \infty} \lim _{m \rightarrow \infty} \Phi_{T}\left(u_{n}, u_{m} ; h_{n}, h_{m}\right)=0 .
$$

Consequentemente, $\Phi_{T}$ é contrativa em $B_{0} \times B_{0}$.

Resumindo, no Teorema 4.5.1 provamos que a família de processos $U_{h}(t, \tau), h \in$ $\mathcal{H}(g)$ admite um conjunto absorvente uniforme limitado $B_{0}$ e é fracamente contínuo em $\mathcal{H} \times \mathcal{H}(g)$. Agora, provamos que dado qualquer $\varepsilon>0$ existe $T=T\left(\varepsilon, B_{0}\right)>0$ e uma função contrativa $\Phi_{T}$ em $B_{0} \times B_{0}$ tal que

$$
\left\|U_{h_{1}}(T, 0) z^{1}-U_{h_{2}}(T, 0) z^{2}\right\|_{\mathcal{H}} \leq \varepsilon+\Phi_{T}\left(z^{1}, z^{2} ; h_{1}, h_{2}\right)
$$

para quaisquer $z^{1}, z^{2} \in B_{0}$ e quaisquer $h_{1}, h_{2} \in \mathcal{H}(g)$, onde

$$
\begin{aligned}
\Phi_{T}\left(z^{1}, z^{2} ; h_{1}, h_{2}\right)= & 2\left\{C_{T, 0} \int_{0}^{T}\left\|u^{1}(s)-u^{2}(s)\right\|_{L^{4}(\Omega)}^{2} d s\right\}^{\frac{1}{2}} \\
& +4\left\{\int_{0}^{T} \int_{\Omega}\left(f\left(u^{2}(s)\right)-f\left(u^{1}(s)\right)\right)\left(u_{t}^{1}(s)-u_{t}^{2}(s)\right) d x d s\right\}^{\frac{1}{2}} \\
& +4\left\{\int_{0}^{T} e^{-\sigma_{1}(T-s)} \int_{\Omega}\left(h_{1}(s)-h_{2}(s)\right)\left(u^{1}(s)-u^{2}(s)\right) d x d s\right\}^{\frac{1}{2}} \\
& +4\left\{\int_{0}^{T} e^{-\sigma_{1}(T-s)} \int_{\Omega}\left(h_{1}(s)-h_{2}(s)\right)\left(u_{t}^{1}(s)-u_{t}^{2}(s)\right) d x d s\right\}^{\frac{1}{2}} .
\end{aligned}
$$

Portanto pelo Teorema 2.4.36 a família de processos $U_{h}(t, \tau), h \in \mathcal{H}(g)$ é uniformemente assitoticamente compacta e assim pelo Teorema 2.4.33 concluímos que o atrator uniforme fraco obtido no Teorema 4.5.1 é na verdade um atrator forte. 


\section{BIBLIOGRAFIA}

[1] R. Adams, J. J. F. Fournier, Sobolev Spaces, $2^{\text {nd }}$ edition, Elsevier/Academic Press, Amsterdam, 2003.

[2] J. M. Arrieta, A. N. Carvalho, J. K. Hale, A damped hyperbolic equation with critical exponent, Comm. Partial Differential Equations 17 (1992) 841-866.

[3] G. Avalos, I. Lasiecka, R. Rebarber, Uniform decay properties of a model in structural acoustics, J. Math. Pures Appl. 79 (2000) 1057-1072.

[4] J. T. Beale, S. I. Rosencrans, Acoustic boundary conditions, Bull Amer. Math. Soc. 80 (1974) 1276-1278.

[5] J. T. Beale, Spectral properties of an acoustic boundary condition, Indiana Univ. Math. J. 25 (1976) 895-917.

[6] J. T. Beale, Acoustic scattering from locally reacting surfaces, Indiana Univ. Math. J. 26 (1977) 199-222.

[7] H. Brezis, Functional analysis, Sobolev spaces and partial differential equations, Universitext, Springer, New York, 2011.

[8] T. Caraballo, A. N. Carvalho, J. A. Langa, J. A. Rivero, Existence of pullback attractors for pullback asymptotically compact processes, Nonl. Anal. 72 (2010) 1967-1976.

[9] T. Caraballo, G. Łukaszewicz, J. Real, Pullback attractors for asymptotically compact non-autonomous dynamical systems, Nonlinear Anal. 64 (2006) 484-498.

[10] A. N. Carvalho, J. A. Langa, J. C. Robinson, Attractors for infinite-dimensional non-autonomous dynamical systems, Applied Mathematical Sciences 182, Springer, New York, 2013. 
[11] M. M. Cavalcanti, V. N. Domingos Cavalcanti, Introdução teoria das distribuições e aos espaços de Sobolev, Maringá: Eduem, (2009).

[12] M. M. Cavalcanti, V. N. Domingos Cavalcanti, V. Komornik, Introdução à análise funcional, Maringá: Eduem, (2011).

[13] M. M. Cavalcanti, L. H. Fatori, T. F. Ma, Attractors for wave equation with degenerate memory, J. Differential Equations 260 (2016) 56-83.

[14] V. V. Chepyzhov, M. I. Vishik, Attractors for Equations of Mathematical Physics, Amer. Math. Soc. Colloq. Publ. Vol. 49, Amer. Math. Soc., Providence, 2002.

[15] G. Chen, J. Duan, J. Zhang, Approximating dynamics of a singularly perturbed stochastic wave equation with a random dynamical boundary condition, SIAM J. Math. Anal. 45 (2013) 2790-2814.

[16] I. Chueshov, M. Eller, I. Lasiecka, On the attractor for a semilinear wave equation with critical exponent and nonlinear boundary dissipation, Comm. Partial Differential Equations 27 (2002) 1901-1951.

[17] I. Chueshov, I. Lasiecka, Long-time dynamics of von Karman semi-flows with nonlinear boundary/interior damping, J. Differential Equations 233 (2007) 42-86.

[18] I. Chueshov, I. Lasiecka, Von Karman evolution equations: Well- posedness and long-time dynamics, Springer, Serie Springer Monographs in Mathematics (2010).

[19] H. Crauel, F. Flandoli, Attractors for random dynamical systems, Probab. Theory Related Fields 100 (1994) 365-393.

[20] M. Efendiev, S. Zelik, A. Miranville, Exponential attractors and finite-dimensional reduction for non-autonomous dynamical systems, Proc. Roy. Soc. Edinburgh Sect. A, 135 (2005) 703-730.

[21] S. Frigeri, Attractors for semilinear damped wave equation with an acoustic boundary condition, J. Evol. Equ. 10 (2010) 29-58.

[22] S. Frigeri, On the convergence to stationary solutions for a semilinear wave equation with an acoustic boundary condition, Z. Anal. Anwend. 30 (2011) 181-191. 
[23] C. L. Frota, J. A. Goldstein, Some nonlinear wave equations with acoustic boundary conditions, J. Differential Equations 164 (2000) 92-109.

[24] C. L. Frota, L. A. Medeiros, A. Vicente, Wave equation in domains with non-locally reacting boundary, Differential Integral Equations 24 (2011) 1001-1020.

[25] C. G. Gal, G. R. Goldstein, J. A. Goldstein, Oscillatory boundary conditions for acoustic wave equations, J. Evol. Equ. 3 (2003) 623-635.

[26] J. García-Luengo, P. Marín-Rubio, J. Real, Pullback attractors in $V$ for nonautonomous 2D-Navier-Stokes equations and their tempered behaviour, J. Differential Equations 252 (2012) 4333-4356.

[27] P. J. Graber, Uniform boundary stabilization of a wave equation with nonlinear acoustic boundary conditions and nonlinear boundary damping, J. Evol. Equ. 12 (2012) 141-164.

[28] P. J. Graber, B. Said-Houari, On the wave equation with semilinear porous acoustic boundary conditions, J. Differential Equations 252 (2012) 4898-4941.

[29] V. Kalantarov, A. Savostianov, S. Zelik, Attractors for damped quintic wave equations in bounded domains, Annales Henri Poincaré 17 (2016) 2555?2584.

[30] Y. Kobayashi, N. Tanaka, An application of semigroups of locally Lipschitz operators to carrier equations with acoustic boundary conditions, J. Math. Anal. Appl. 338 (2008) 852-872.

[31] J. L. Lions, Quelques Méthodes de Résolution des Problèmes aux Limites non Linéaries, Dunod Gauthier-Villars, Paris, (1969).

[32] S. Ma, X. Cheng, H. Li, Attractors for non-autonomous wave equations with a new class of external forces, J. Math. Anal. Appl. 337 (2008) 808-820.

[33] T. F. Ma, P. Marin-Rubio, C. Surco-Chuño, Dynamics of wave equations with moving boundary, preprint 2015.

[34] T. F. Ma, T. M. Souza, Pullback dynamics of non-autonomous wave equations with acoustic boundary condition, Differential Integral Equations. (a aparecer) 
[35] S. Ma, C. Zhong, The attractors for weakly damped non-autonomous hyperbolic equations with a new class of external forces, Discrete Contin. Dyn. Syst. 18 (2007) 53-70.

[36] A. Miranville, S. Zelik, Attractors for dissipative partial differential equations in bounded and unbounded domains, in Handbook of Differential Equations: Evolutionary Equations. Vol. IV, Handb. Differ. Equ., Elsevier/North-Holland, Amsterdam, (2008) 103-200.

[37] P. M. Morse, K. U. Ingard, Theoretical Acoustics, McGraw-Hill, New York, (1968).

[38] D. Mugnolo, Abstract wave equation with acoustic boundary conditions, Math. Nachr. 279 (2006) 299-318.

[39] M. Renardy, R. C. Rogers, An Introduction to Partial Differential Equations, Springer-Verlag New York, 13 (2004).

[40] J. Simon, Compact sets in the space $L^{p}(0, T ; B)$, Ann. Mat. Pura Appl. 146 (1987) 65-96.

[41] C. Sun, D. Cao, J. Duan, Non-autonomous dynamics of wave equation with nonlinear damping and critical nonlinearity, Nonlinearity 19 (2006) 2645-2665.

[42] C. Sun, D. Cao, J. Duan, Uniform attractors for nonautonomous wave equations with nonlinear damping, SIAM J. Appl. Dyn. Syst. 6 (2007) 293-318.

[43] A. Vicente, C. L. Frota, Nonlinear wave equation with weak dissipative term in domains with non-locally reacting boundary, Wave Motion 50 (2013) 162-169.

[44] Y. Wang, On the upper semicontinuity of pullback attractors with applications to plate equations, Commun. Pure Appl. Anal. 9 (2010) 1653-1673.

[45] S. Zelik, Strong uniform attractors for non-autonomous dissipative PDEs with non translation-compact external forces, Discrete and Continuous Dynamical Systems B 20 (2015) 781-810. 